\title{
Unveiling the high catalytic activity of a dinuclear iron complex for oxygen evolution reaction
}

\author{
Shaojin Hu, ${ }^{1}$ Penglin Xu, ${ }^{1}$ Rui-Xue Xu, ${ }^{1,2}$ and Xiao Zheng ${ }^{* 1,2}$
}

${ }^{1}$ Hefei National Laboratory for Physical Sciences at the Microscale \& Synergetic Innovation Center of Quantum Information and Quantum Physics \& CAS Center for Excellence in Nanoscience, University of Science and Technology of China, Hefei, Anhui 230026, China

${ }^{2}$ Department of Chemical Physics \& Key Laboratory of Surface and Interface Chemistry and Energy Catalysis of Anhui Higher Education Institutes, University of Science and Technology of China, Hefei, Anhui 230026, China

*Email: xz58@ustc.edu.cn 
This supplemental file includes the following materials:

Figure S1. The optimized structures of the aqueous Ce ions with explicit and implicit solvation models, together with the corresponding computational methods.

Figure S2. The optimized structure of the Fe ${ }^{\mathrm{III}}(\mathrm{ppq})$ complex.

Figure S3. The proton coupled electron transfer (PCET) process from $\mathrm{Fe}^{\mathrm{III}}(\mathrm{ppq})$ complex to complex 1.

Table S1. The relative energy of complex 1 in different spin states.

Figure S4. The optimized structures of the TSs and the energy diagrams for the nitrate and water attack mechanism of the complex $\mathbf{1}$.

Figure S5. The Mulliken charge differences between the complex 1' and the complex $\mathbf{1}$.

Figure S6. The optimized structures of the complex 1' and the TS1 $1^{\prime}$ and the energy diagram for the nitrate nucleophilic attack pathway.

Figure S7. The imaginary-frequency vibrational mode of the (TS1 1 ).

Figure S8. The optimized structures of the Int11, complex 2 and complex 3.

Figure S9. The PCET process of the complex 2, from the $\left[\mathrm{H}_{2} \mathrm{O}-\mathrm{Fe}^{\mathrm{III}}-\mathrm{OH}_{2}\right]^{3+}$ to the $\left[\mathrm{H}_{2} \mathrm{O}-\mathrm{Fe}^{\mathrm{V}}-\mathrm{O}\right]^{3+}$ state.

Figure S10. The optimized structures of the complex 4 and complex 5.

Figure S11. The optimized structures of the Int14, the TS24, the Int15 and TS25.

Figure S12. The optimized structures of the Int24 and Int25.

Figure S13. The optimized structure of the $\mathbf{T S 3}_{4}$ and the energy diagram for the water nucleophilic attack pathway of the complex 4 .

Figure S14. The PCET process of the complex 3, from the $\left[\mathrm{Cl}-\mathrm{Fe}^{\mathrm{III}}-\mathrm{OH}_{2}\right]^{2+}$ to the $\left[\mathrm{Cl}-\mathrm{Fe}^{\mathrm{V}}-\mathrm{O}\right]^{2+}$.

Figure S15. The optimized structures of the Int34, Int4 4 , Int3 5 and Int45.

Figure S16. The optimized structures of the Int1 $6_{6}$ and TS2 6 . 
(a)
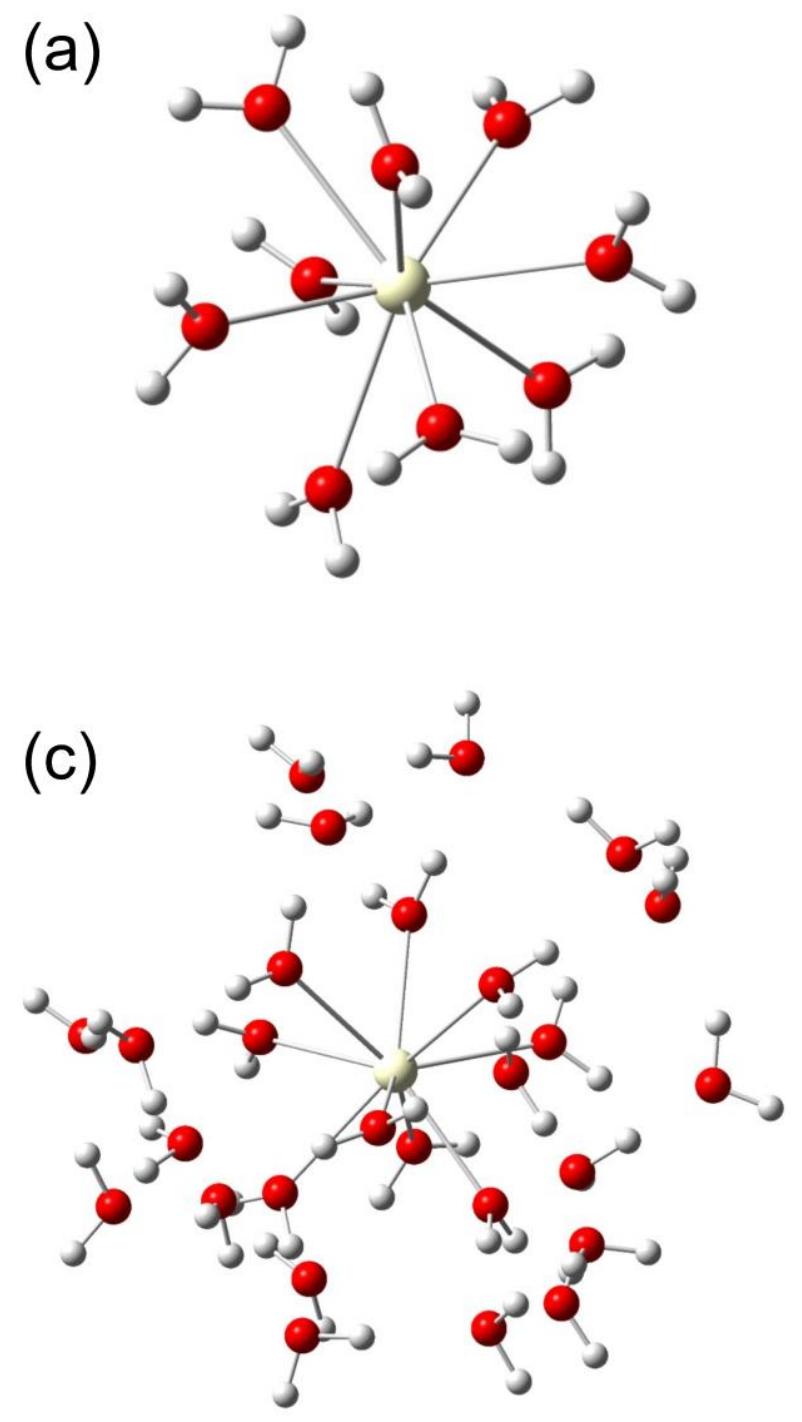

(b)
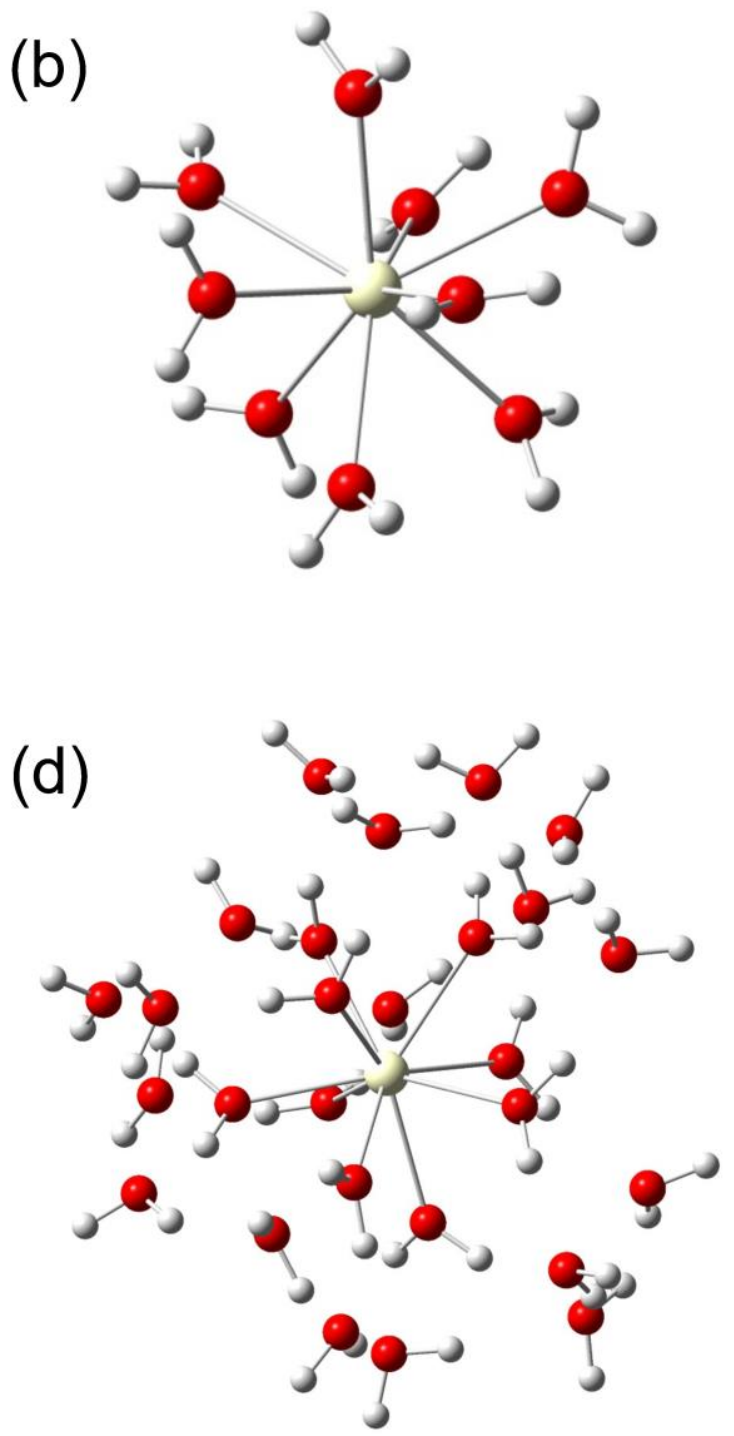

Figure S1. The optimized structures of the (a) $\mathrm{Ce}^{3+}$ ion and (b) $\mathrm{Ce}^{4+}$ ion with the first solvation shell consisting of nine explicit water molecules. The average bond length of the $\left[\mathrm{Ce}\left(\mathrm{H}_{2} \mathrm{O}\right)_{9}\right]^{3+}$ and $\left[\mathrm{Ce}\left(\mathrm{H}_{2} \mathrm{O}\right)_{9}\right]^{4+}$ are $2.57 \AA$ and $2.45 \AA$, respectively. (c) The optimized structures of the $\mathrm{Ce}^{3+}$ ion and (d) $\mathrm{Ce}^{4+}$ ion with the first and second solvation shells consisting of 9 and 18 water molecules, respectively. The yellow, red and white balls represent $\mathrm{Ce}, \mathrm{O}$ and $\mathrm{H}$ atoms, respectively. 
To compare the redox potential of the iron-based complexes to that of the $\mathrm{Ce}^{4+} / \mathrm{Ce}^{3+}$ pair at the same level, a combined explicit and implicit solvation model is adopted for the aqueous $\mathrm{Ce}$ ions, with the first solvation shell consisting of nine explicit water molecules (Figure S1(a) and(b)). ${ }^{1}$ The absolute redox potential of the $\mathrm{Ce}^{4+} / \mathrm{Ce}^{3+}$ redox pair is calculated by the $\omega \mathrm{B} 97 \mathrm{XD}$ functional. ${ }^{2}$ A mixed basis set, consisting of the $\mathrm{SDD}^{3}$ basis set for the Ce and the $6-31 \mathrm{G}(\mathrm{d}, \mathrm{p})$ basis set for the $\mathrm{O}$ and $\mathrm{H}$, is adopted for the geometry optimization and frequency calculations. The final and solvation energies in the aqueous are calculated by the $\mathrm{SMD}^{4}$ solvation model with SDD basis set for the Ce atom and 6-311+G(2df,2p) for all other atoms. The redox potential of $\mathrm{Ce}^{4+} / \mathrm{Ce}^{3+}$ pair is calculated to be $3.07 \mathrm{~V}$, which is taken as a reference potential for all redox steps.

In order to consider the influence of the outer water molecules on the solvated Ce ions, we further add the second layer of water molecules (18 water molecules) in our calculation model (Figure S1(c) and (d)). Due to the complexity of the calculation model, the HF method is used to optimize the structure. A mixed basis set, consisting of the $\mathrm{SDD}^{3}$ basis set for the $\mathrm{Ce}$ and the 6$31 \mathrm{G}^{*}$ basis set for the $\mathrm{O}$ and $\mathrm{H}$, is adopted for the geometry optimization and frequency calculations. The final and solvation energies in the aqueous are calculated by $\omega$ B97XD functional with the SDD pseudopotential for the Ce atom, as well as $6-311+\mathrm{G}(2 \mathrm{df}, 2 \mathrm{p})$ for all other atoms. The SMD continuum solvation model is adopted for considering the aqueous solvent. In this case, the redox potential of the $\mathrm{Ce}^{4+} / \mathrm{Ce}^{3+}$ pair is calculated to be $2.70 \mathrm{~V}$, which is lower than the redox potential when only the first water solvation shell is treated explicitly. 


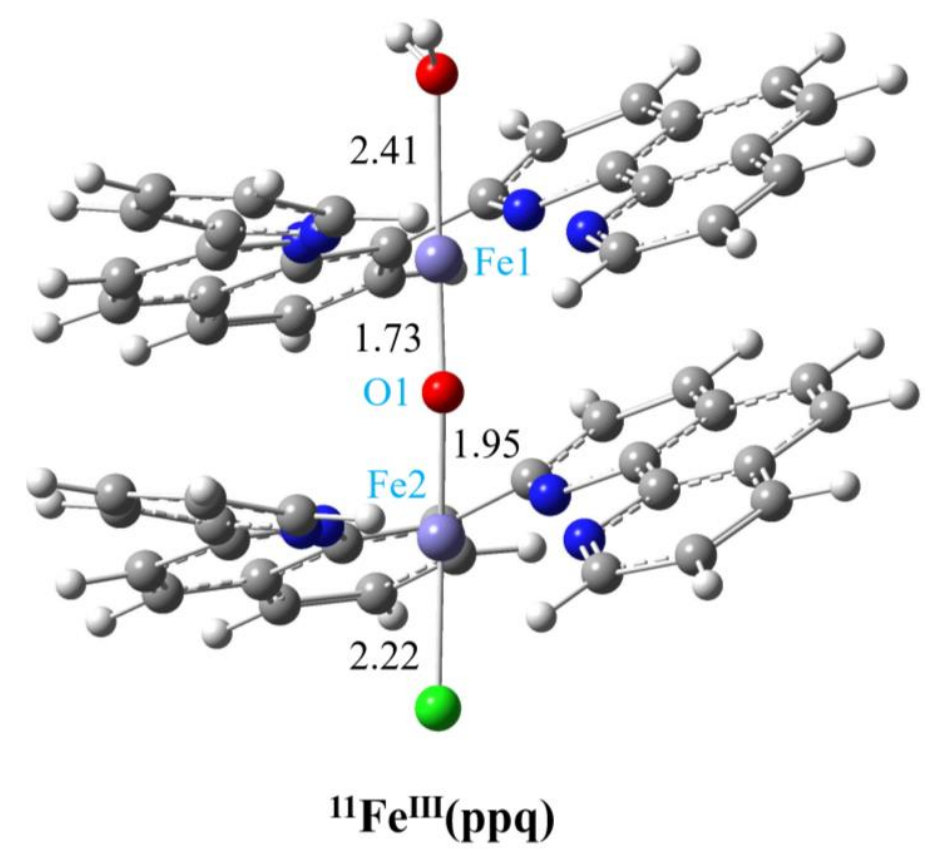

Figure S2. The optimized structure of the $\mathrm{Fe}^{\mathrm{III}}(\mathrm{ppq})$ complex. Some interatomic distances are shown by numbers in black (in units of $\AA$ ). The purple, red, blue, gray and white balls represent $\mathrm{Fe}, \mathrm{O}, \mathrm{N}, \mathrm{C}$, and $\mathrm{H}$ atoms, respectively. Left superscripts denote the multiplicity of the species

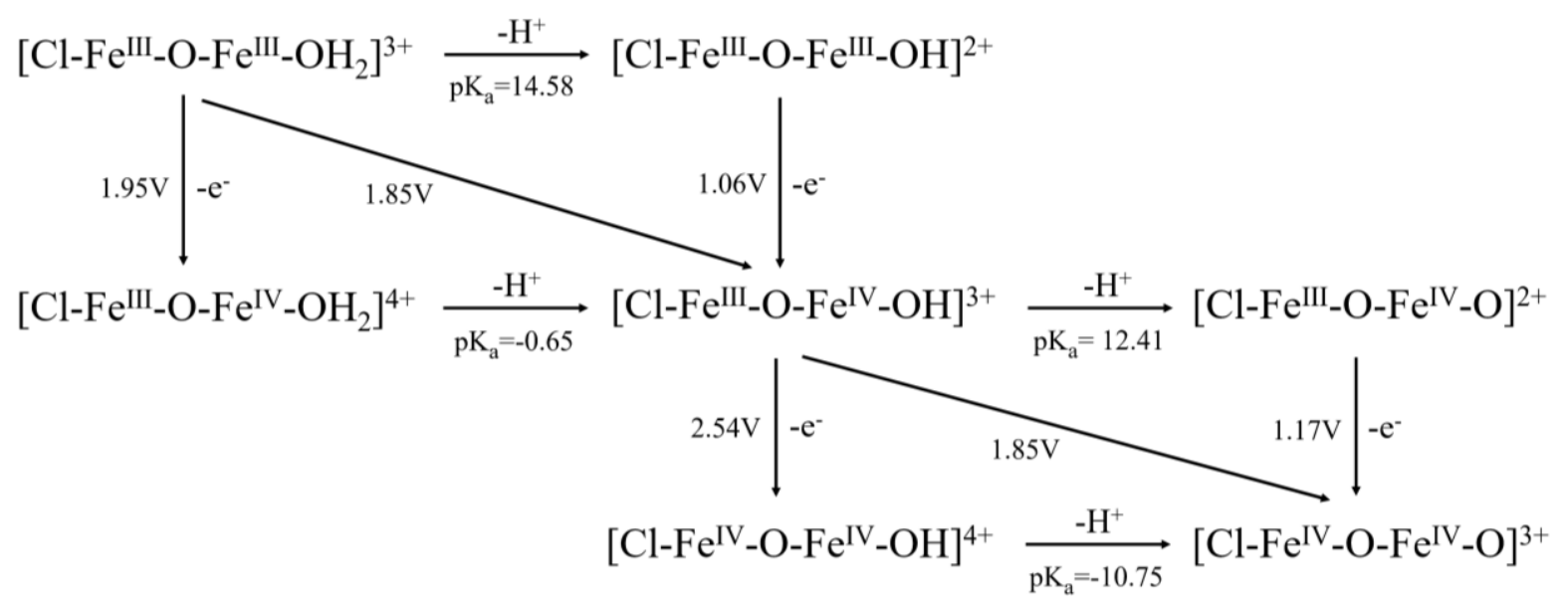

Figure S3. The proton coupled electron transfer (PCET) process from the $\left[\mathrm{Cl}-\mathrm{Fe}^{\mathrm{III}}-\mathrm{O}-\mathrm{Fe}^{\mathrm{III}}-\mathrm{OH}_{2}\right]^{3+}$ to $\left[\mathrm{Cl}-\mathrm{Fe}^{\mathrm{IV}}-\mathrm{O}-\mathrm{Fe}^{\mathrm{IV}}-\mathrm{O}\right]^{3+}$. The ppq is omitted for brevity. 


\begin{tabular}{|l|r|r|r|r|r|r|}
\hline \multicolumn{7}{|c|}{ different functionals(kcal/mol) } \\
\hline Spin multiplicity & $\mathbf{1}$ & $\mathbf{3}$ & $\mathbf{5}$ & $\mathbf{7}$ & $\mathbf{9}$ & $\mathbf{1 1}$ \\
\hline WB97XD functional & 53.7 & 8.8 & 0.0 & 4.8 & 22.2 & 15.2 \\
\hline PBE functional & 4.5 & 7.3 & 0.0 & 13.6 & 26.9 & 35.1 \\
& & & & & & \\
\hline
\end{tabular}

As shown in Table S1, the triplet, quintuple, and heptad states are the three lowest energy spin states of complex 1 by using $\omega \mathrm{B} 97 \mathrm{XD}$ functional. Therefore, we only consider these three spin states in the mechanism study. The $\omega \mathrm{B} 97 \mathrm{XD}$ and PBE functionals both indicate that the ground state of the complex $\mathbf{1}$ is the quintet state. The energies of these different spin states are all calculated at the optimized geometries. 

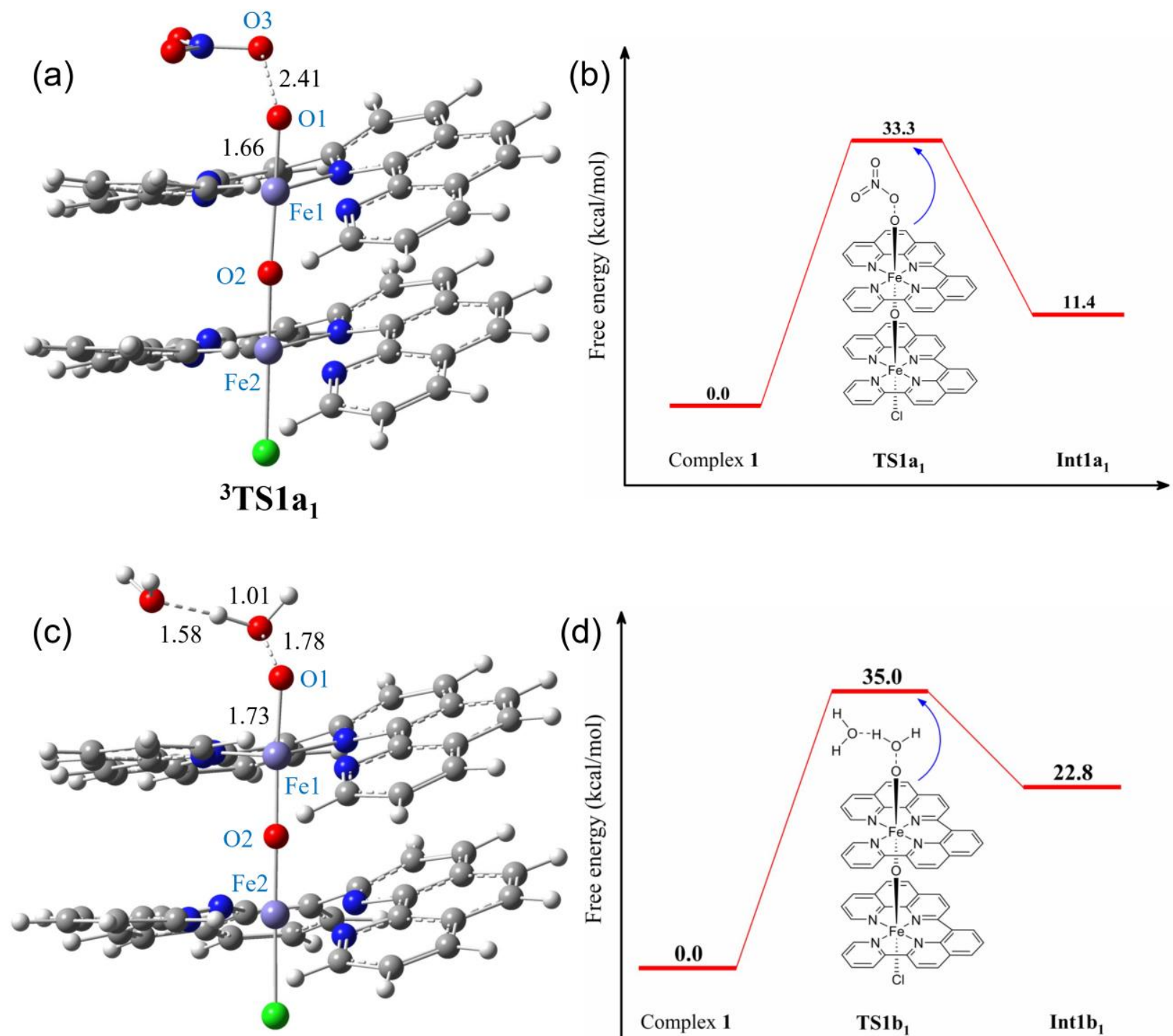

${ }^{3} \mathrm{TS} \mathrm{b}_{1}$

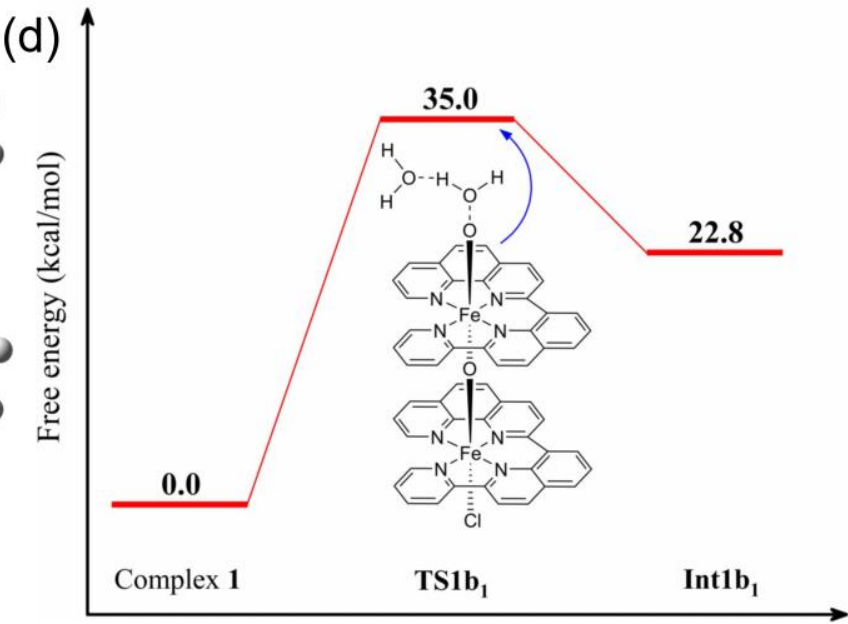

Figure S4. (a) The optimized structure of the TS1a1 for the nitrate nucleophilic attack mechanism.

(b) Energy diagram for the nitrate attack pathway. (c) The optimized structure of the TS1 $\mathbf{b}_{1}$ for the water nucleophilic attack pathway. (d) Energy diagram for the water nucleophilic attack pathway. Some interatomic distances are shown by numbers in black (in units of $\AA$ ). The purple, red, blue, gray and white balls represent $\mathrm{Fe}, \mathrm{O}, \mathrm{N}, \mathrm{C}$, and $\mathrm{H}$ atoms, respectively. 


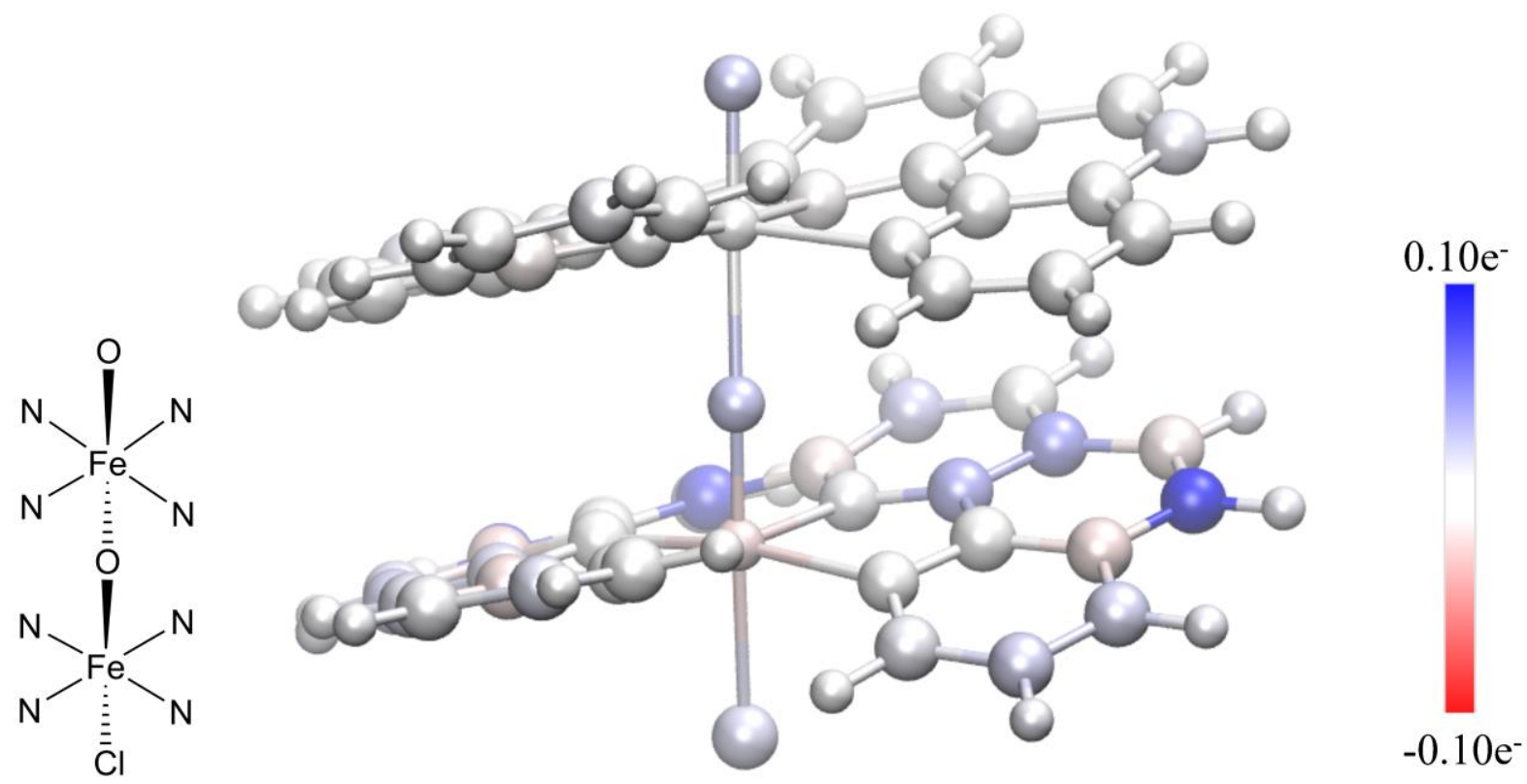

Figure S5. The natural atomic charge differences between the complex $\mathbf{1}^{\prime}$ and the complex $\mathbf{1}$. 


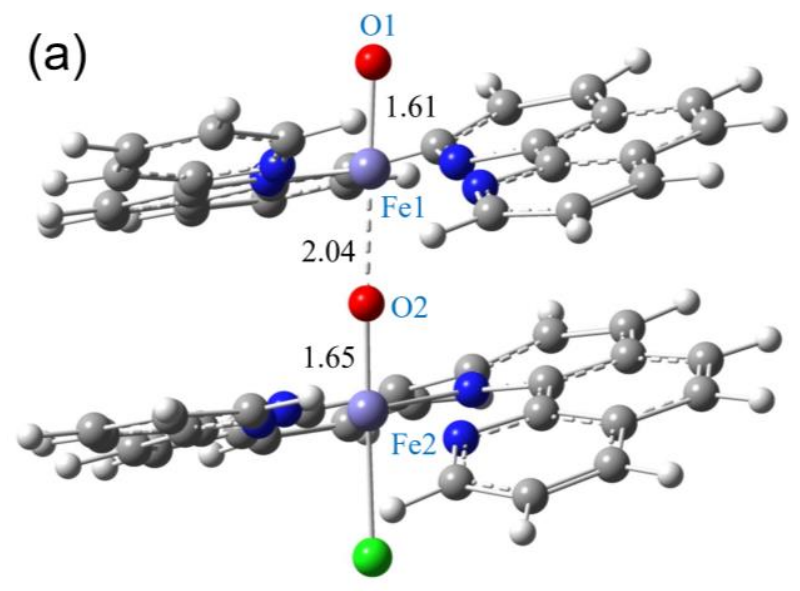

${ }^{6} 1^{\prime}$

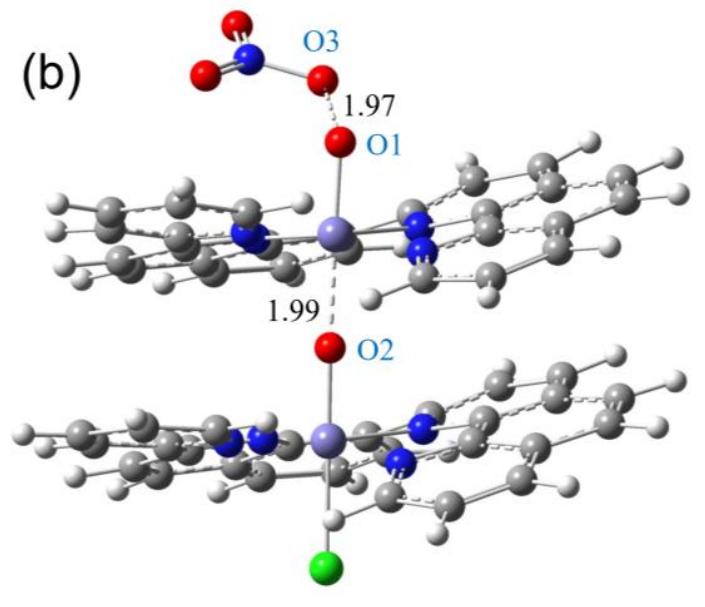

${ }^{4} \mathrm{TS1}_{1}{ }^{\prime}$

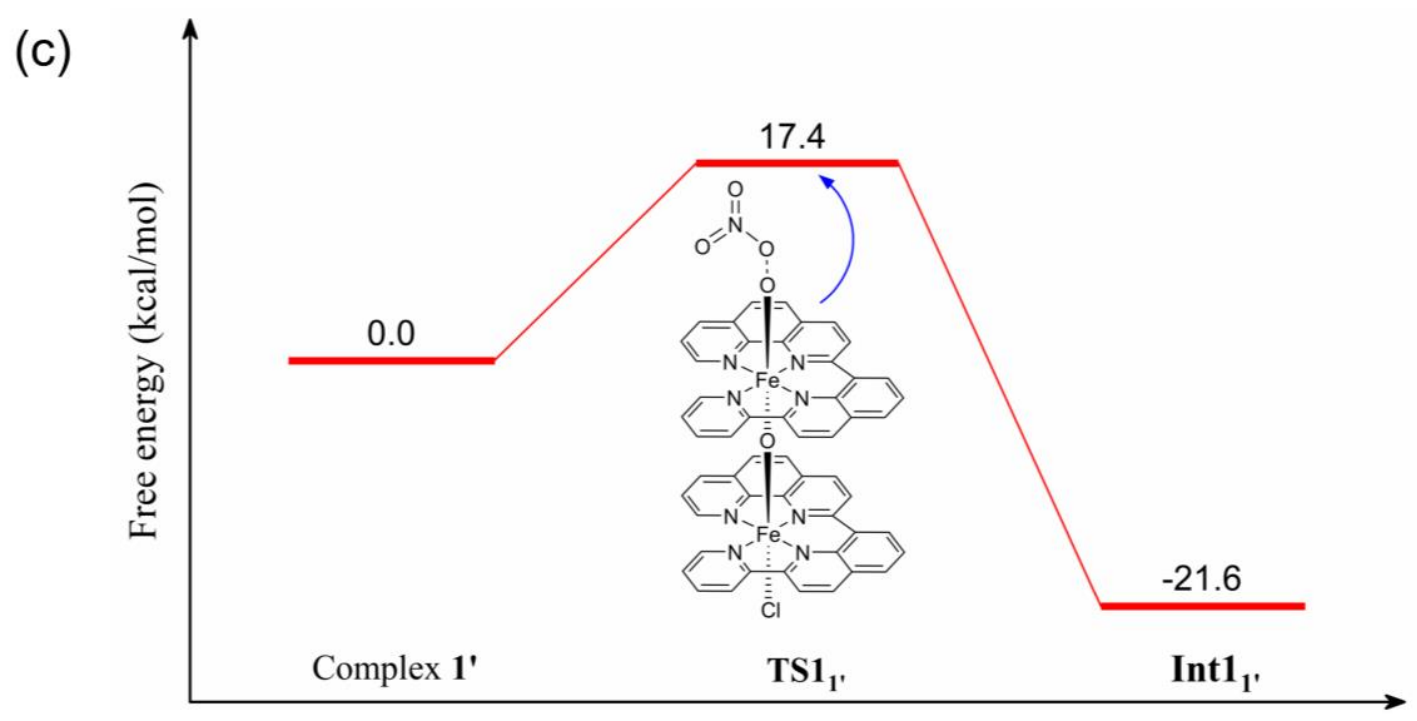

Figure S6. (a) The optimized structures of the complex 1' (b) and the TS11' for the nitrate nucleophilic attack pathway. (c) Energy diagram for the nitrate nucleophilic attack pathway. The purple, red, blue, gray and white balls represent Fe, O, N, C, and $\mathrm{H}$ atoms, respectively. 


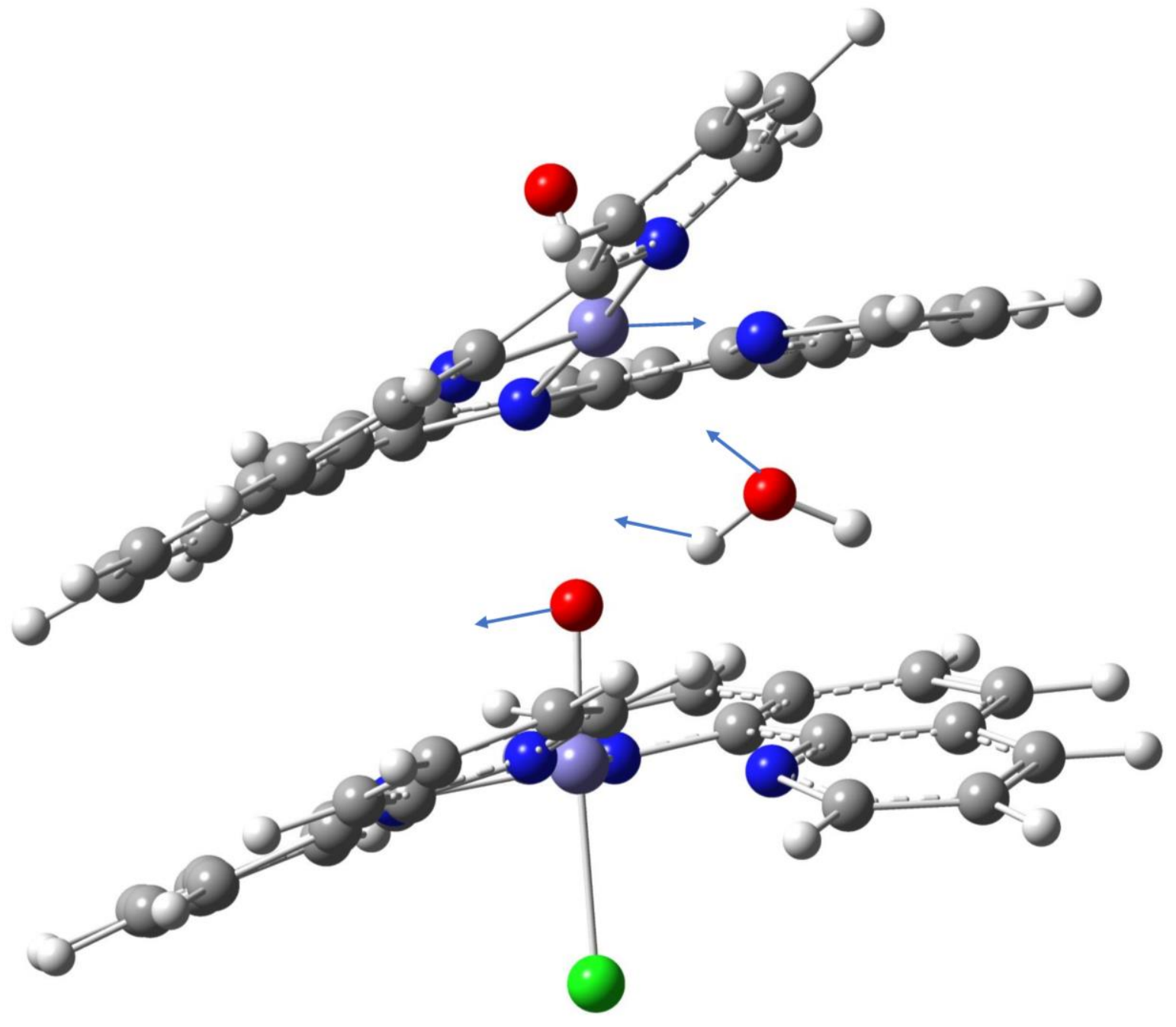

Figure S7. The optimized structure of the TS11. The arrows indicate the imaginary-frequency vibrational mode of the transition state. A supplemental file [movie.mp4] showing the animation of this vibrational mode is also provided. 


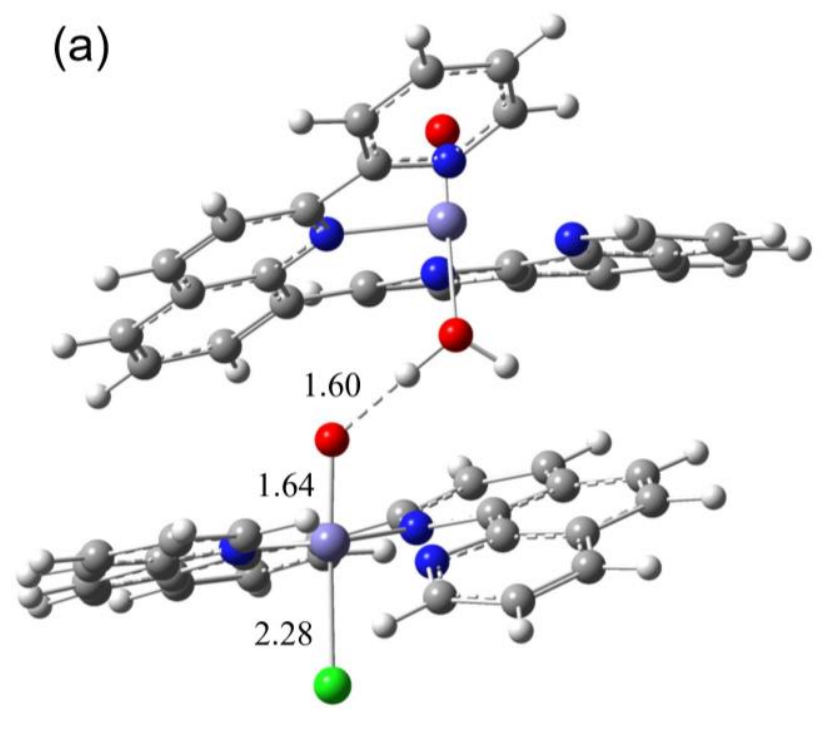

${ }^{5}$ Int1 $_{1}$ (b)

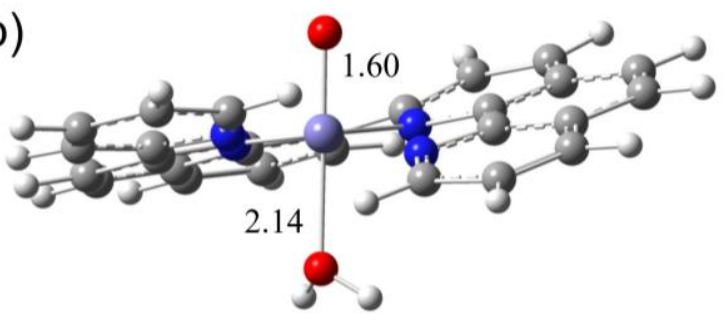

32

(c)

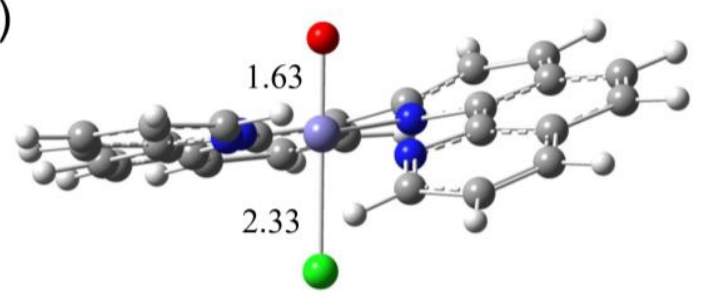

33

Figure S8. The optimized structures of the (a) Int11, (b) complex 2 and (c) complex 3. Some interatomic distances are shown by numbers in black (in units of $\AA$ ).

When Int1 1 is separated into complexes $\mathbf{2}$ and $\mathbf{3}$, the water ligand and $\mathrm{O}$ ligand between the two ppq ligands in Int11 will be exposed to the solution. Therefore, these ligands will form hydrogen bonds with water molecules in the solution to reduce their energy and make it more stable. The relative energies of Int1 1 and complexes $\mathbf{2}$ and $\mathbf{3}$ in the main text are obtained by considering the effect of these hydrogen bonds. 


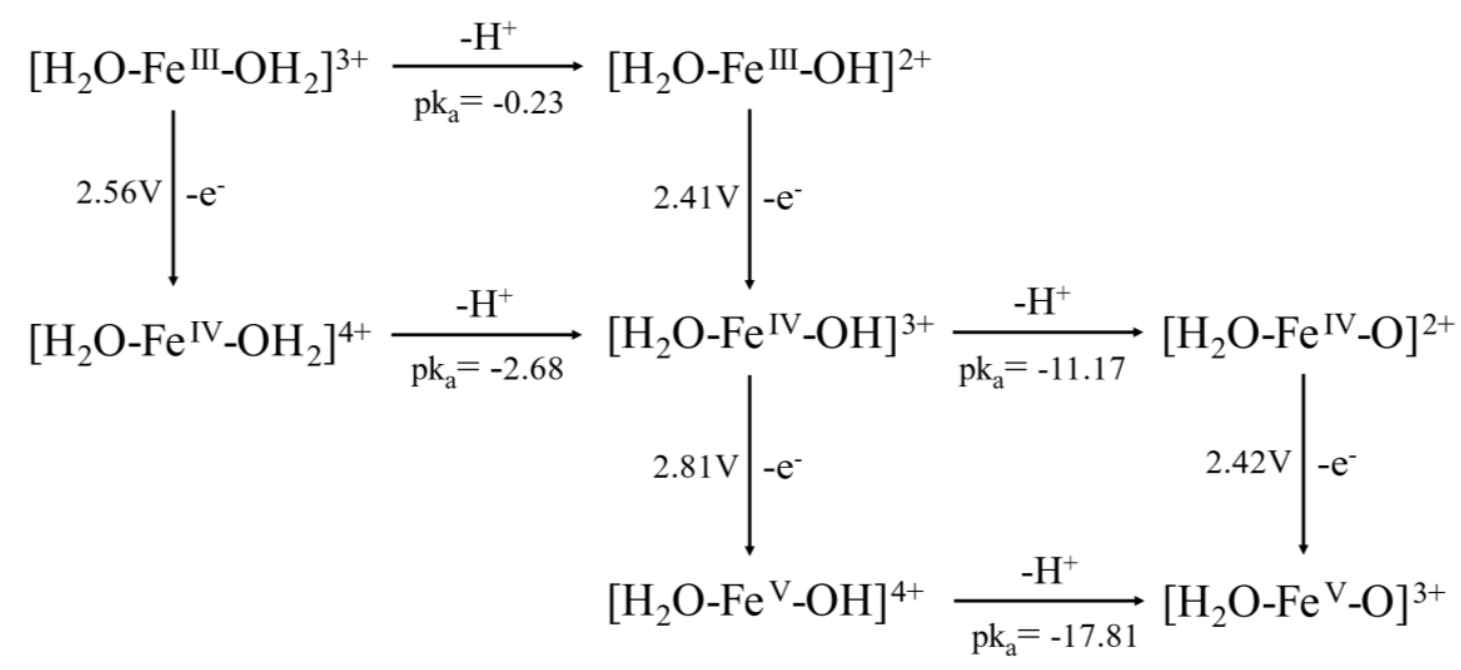

Figure S9. The PCET process of the complex 2, from the $\left[\mathrm{H}_{2} \mathrm{O}-\mathrm{Fe}^{\mathrm{III}}-\mathrm{OH}_{2}\right]^{3+}$ to the $\left[\mathrm{H}_{2} \mathrm{O}-\mathrm{Fe}^{\mathrm{V}}\right.$ $\mathrm{O}]^{3+}$ state.

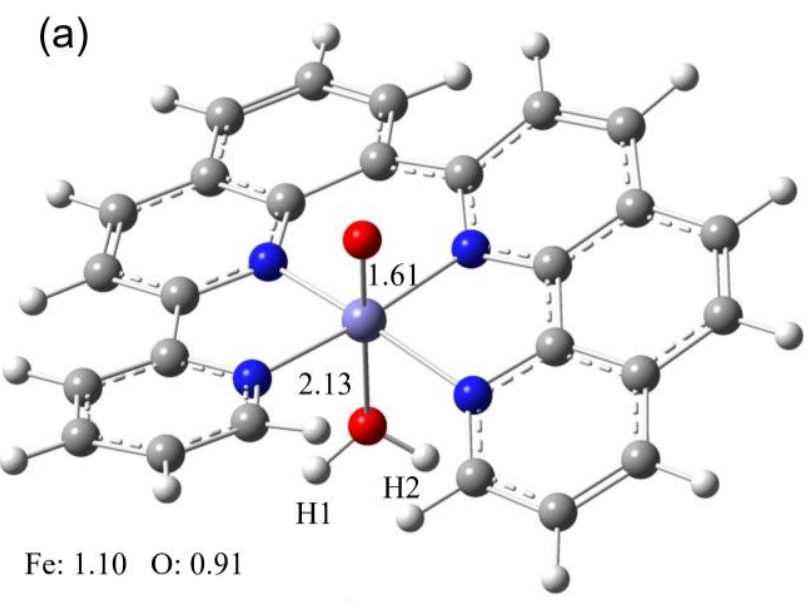

${ }^{4} 4$

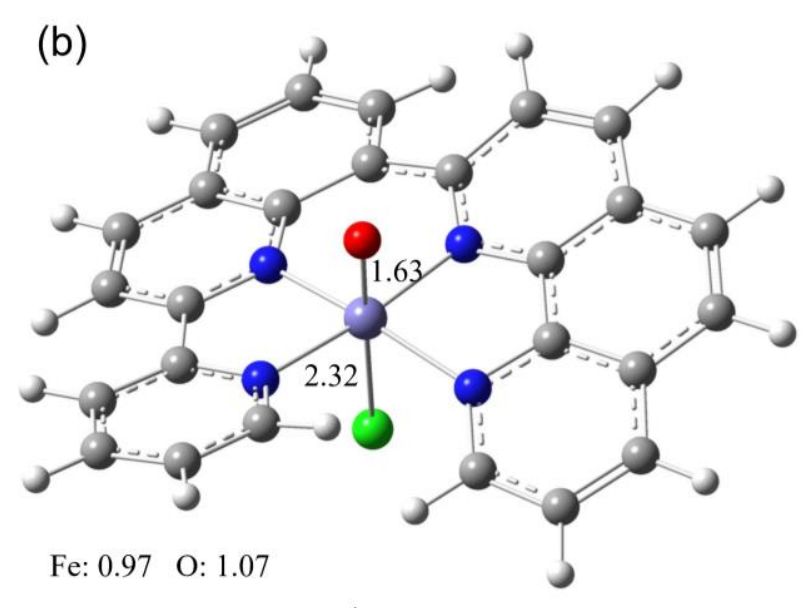

45

Figure S10. The optimized structures of the (a) complex 4 and (b) complex 5. Some interatomic distances are shown by numbers in black (in units of $\AA$ ). Mulliken spin densities on selected atoms are indicated in the lower-left corner of (a) and (b). The pKa of the complex 4 in the text is calculated from the loss of $\mathrm{H} 1$. 


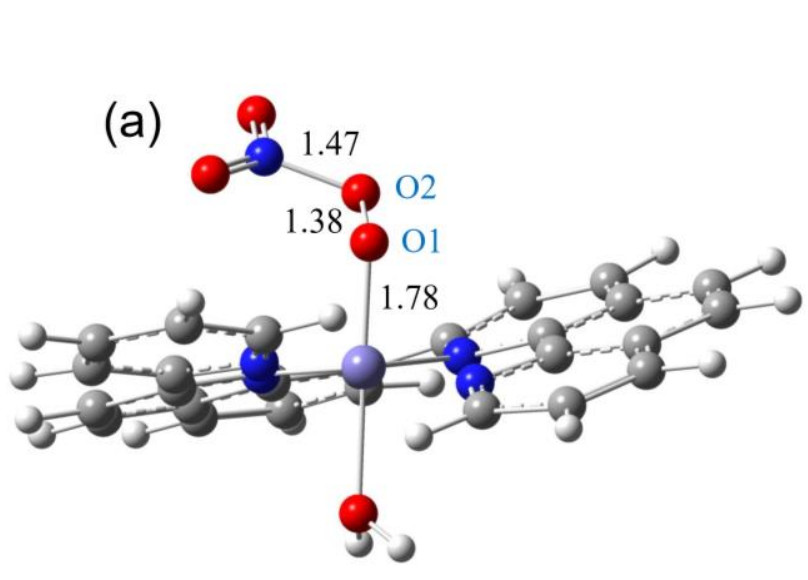

${ }^{2}$ Int1 $1_{4}$

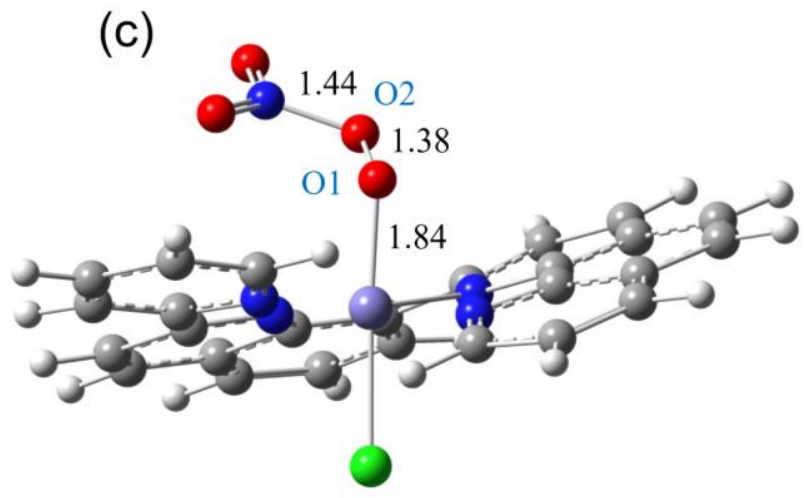

${ }^{2}$ Int1 ${ }_{5}$

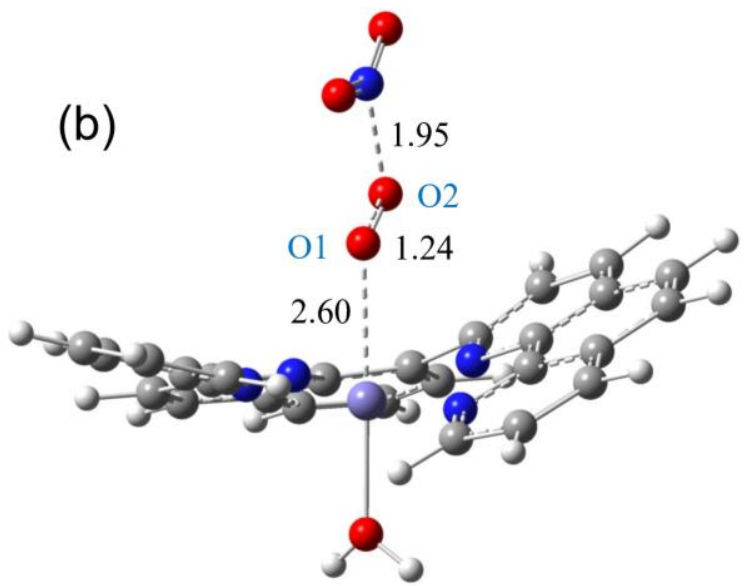

${ }^{6} \mathrm{TS2}_{4}$

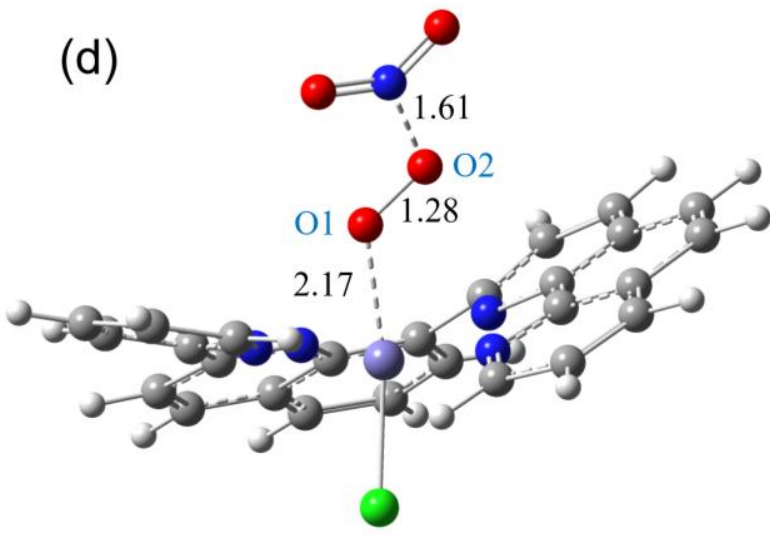

${ }^{6} \mathrm{TS2}_{5}$

Figure S11. The optimized structures of (a) the Int14, (b) the TS2 4 , (c) the Int15 and (d) TS25. Some interatomic distances are shown by numbers in black (in units of $\AA$ ). The purple, red, blue, gray and white balls represent $\mathrm{Fe}, \mathrm{O}, \mathrm{N}, \mathrm{C}$, and $\mathrm{H}$ atoms, respectively. 


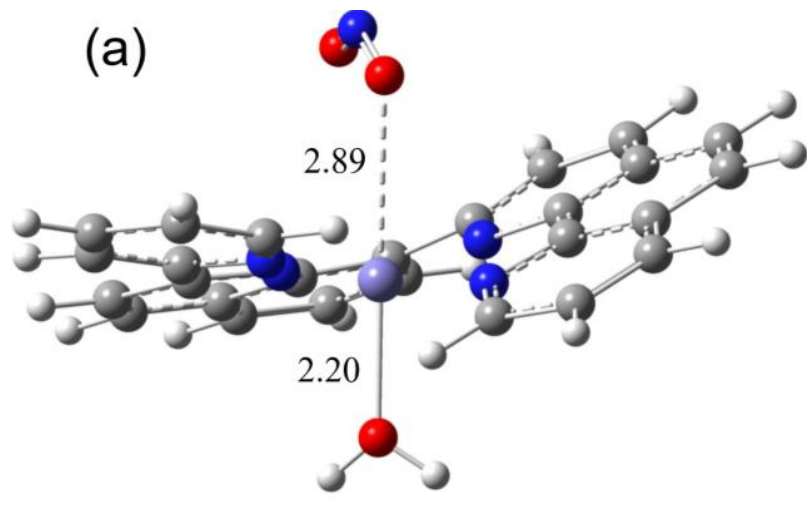

${ }^{4}$Int2 $_{4}$

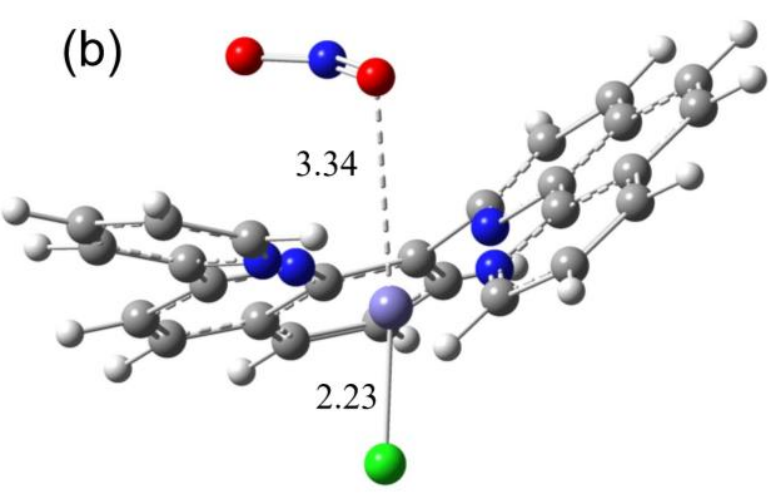

${ }^{6}$ Int2 ${ }_{5}$

Figure S12. The optimized structures of the (a) Int24 and (b) Int25. Some interatomic distances are shown by numbers in black (in units of $\AA$ ).
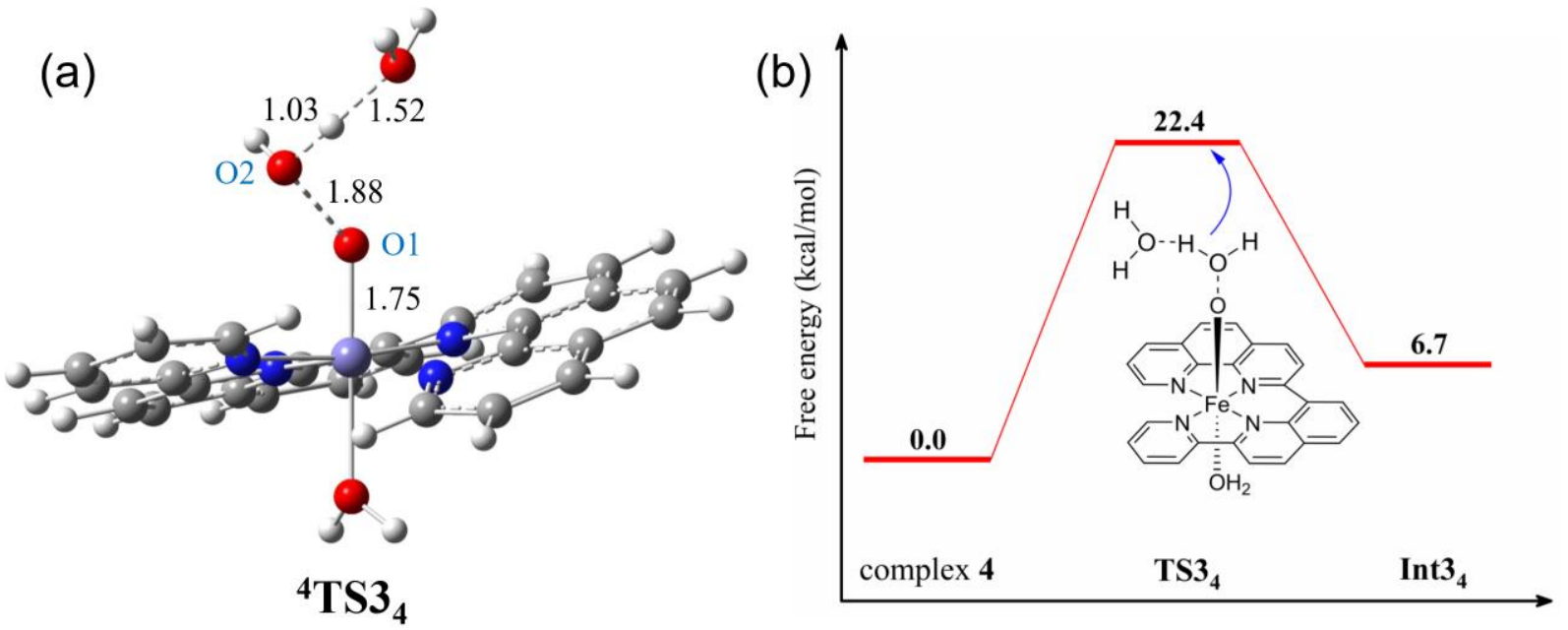

Figure S13. (a) The optimized structure of the $\mathbf{T S 3}_{4}$ for the water nucleophilic attack pathway. (b) Energy diagram for the water nucleophilic attack pathway of the complex 4. Some interatomic distances are shown by numbers in black (in units of $\AA$ ). 


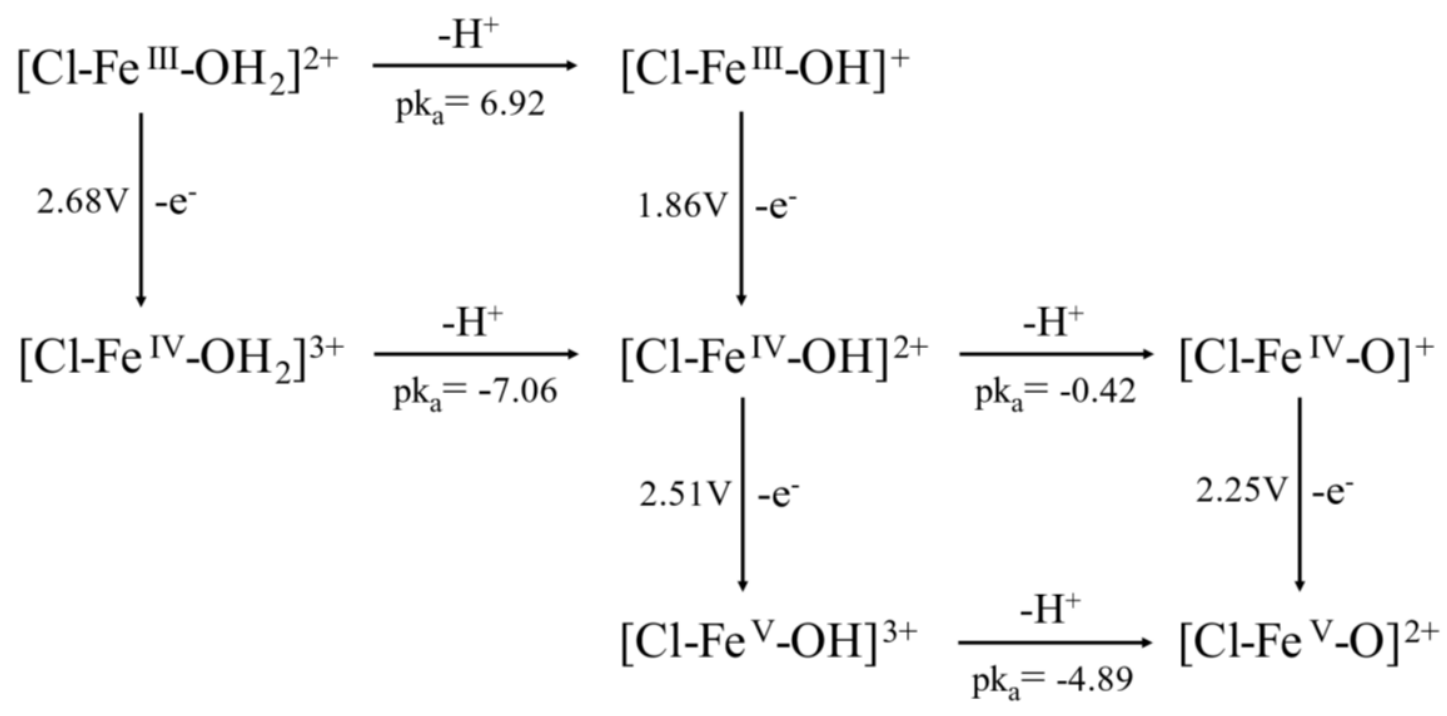

Figure S14. The PCET process of the complex 3, from the $\left[\mathrm{Cl}-\mathrm{Fe}^{\mathrm{III}}-\mathrm{OH}_{2}\right]^{2+}$ to the $\left[\mathrm{Cl}-\mathrm{Fe}^{\mathrm{V}}-\mathrm{O}\right]^{2+}$.

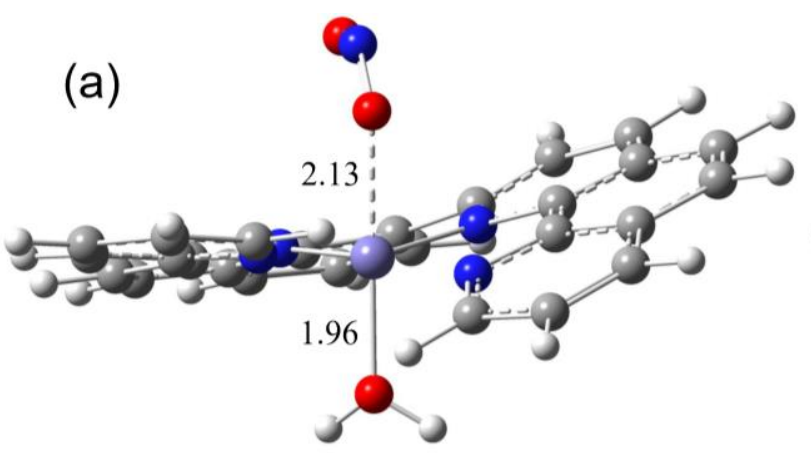

${ }^{3} \operatorname{Int}_{4}$

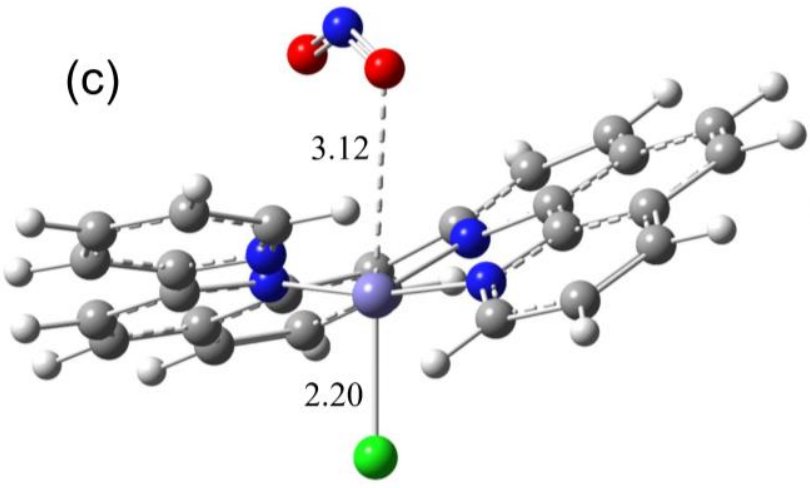

${ }^{5} \operatorname{Int}_{5}$

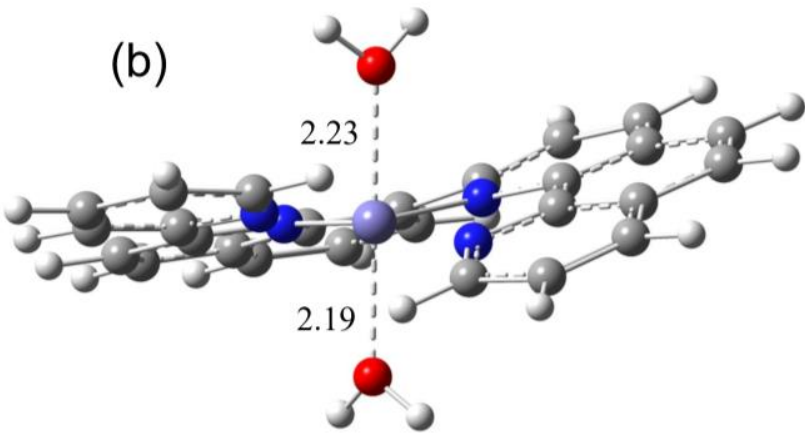

${ }^{4}$Int4$_{4}$

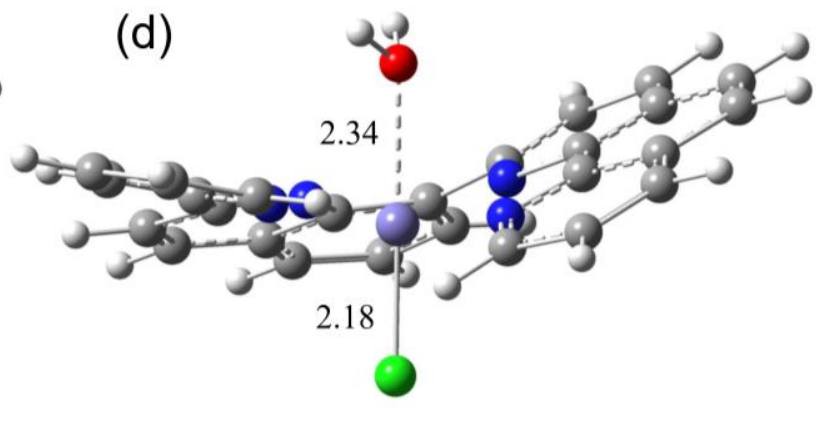

${ }^{6}$ Int4 $_{5}$

Figure S15. The optimized structures of the (a) Int34, (b) Int44, (c) Int35 and (d) Int45. 


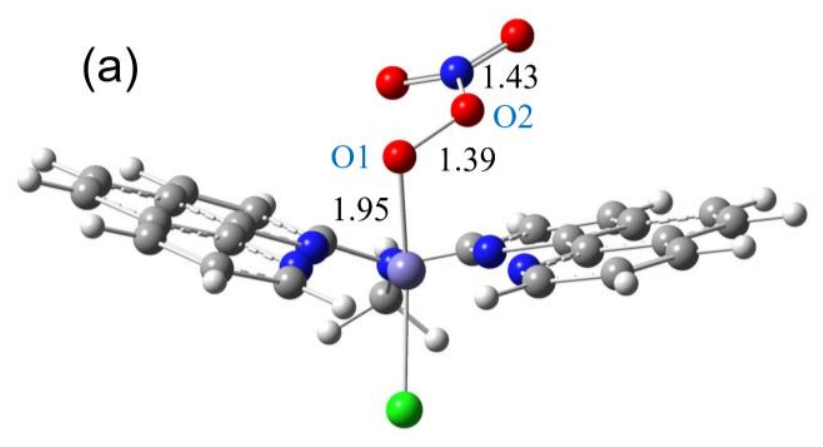

${ }^{6} \operatorname{Int1}_{6}$

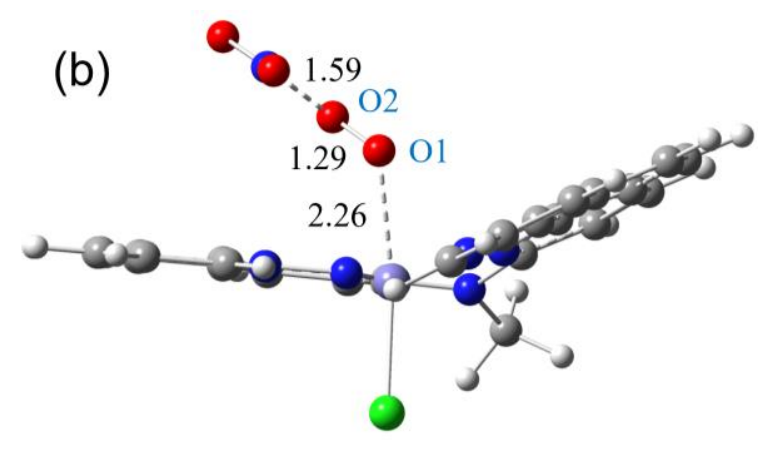

${ }^{6} \mathrm{TS2}_{6}$

Figure S16. The optimized structures of the (a) Int1 ${ }_{6}$ and (b) TS2 6 . Some interatomic distances are shown by numbers in black (in units of $\AA$ ). 


\begin{tabular}{|c|c|c|c|}
\hline \multicolumn{4}{|c|}{$\begin{array}{l}\text { Cartesian coordinates: } \\
\mathrm{Fe}^{\mathrm{III}}(\mathrm{ppq})\end{array}$} \\
\hline \multicolumn{4}{|l|}{311} \\
\hline $\mathrm{Fe}$ & 6.12019500 & 10.94135800 & 3.76146500 \\
\hline $\mathrm{Cl}$ & 7.21954900 & 12.86651400 & 3.57432400 \\
\hline $\mathrm{N}$ & 7.00120000 & 10.60367600 & 5.71036000 \\
\hline $\mathrm{C}$ & 8.26853100 & 10.38944200 & 6.02049900 \\
\hline $\mathrm{H}$ & 8.99980100 & 10.56525400 & 5.23695800 \\
\hline $\mathrm{N}$ & 4.48557000 & 11.36122000 & 5.07357900 \\
\hline $\mathrm{C}$ & 8.66483900 & 9.98129400 & 7.30463000 \\
\hline $\mathrm{H}$ & 9.71634700 & 9.83099600 & 7.51847100 \\
\hline $\mathrm{N}$ & 4.77739700 & 11.43815300 & 2.10935200 \\
\hline $\mathrm{C}$ & 7.70225900 & 9.79903900 & 8.27190300 \\
\hline $\mathrm{H}$ & 7.97835800 & 9.49014800 & 9.27567800 \\
\hline $\mathrm{N}$ & 7.12029000 & 10.08645800 & 2.06133600 \\
\hline $\mathrm{C}$ & 6.34632400 & 10.05209400 & 7.96733500 \\
\hline $\mathrm{C}$ & 6.04890700 & 10.47300100 & 6.66060100 \\
\hline $\mathrm{C}$ & 5.28427900 & 9.92174800 & 8.92408000 \\
\hline $\mathrm{H}$ & 5.52598600 & 9.59604400 & 9.93067400 \\
\hline $\mathrm{C}$ & 4.00617300 & 10.22830700 & 8.58602000 \\
\hline $\mathrm{H}$ & 3.20675800 & 10.14478000 & 9.31493200 \\
\hline $\mathrm{C}$ & 3.69140000 & 10.71340700 & 7.27308800 \\
\hline $\mathrm{C}$ & 4.70264400 & 10.84005300 & 6.31085600 \\
\hline $\mathrm{C}$ & 2.39423800 & 11.10095500 & 6.87918800 \\
\hline $\mathrm{H}$ & 1.56424100 & 10.99060500 & 7.57042300 \\
\hline $\mathrm{C}$ & 2.19459600 & 11.63624900 & 5.63417300 \\
\hline $\mathrm{H}$ & 1.19971900 & 11.93726500 & 5.33384400 \\
\hline $\mathrm{C}$ & 3.28215900 & 11.80999800 & 4.73811800 \\
\hline $\mathrm{C}$ & 3.05813800 & 12.54659300 & 3.46679300 \\
\hline $\mathrm{C}$ & 2.09635200 & 13.54349400 & 3.50761100 \\
\hline $\mathrm{H}$ & 1.63261500 & 13.79132500 & 4.45553900 \\
\hline $\mathrm{C}$ & 1.70416800 & 14.28945800 & 2.38106200 \\
\hline $\mathrm{H}$ & 0.95541400 & 15.06610800 & 2.48563100 \\
\hline $\mathrm{C}$ & 2.27782800 & 14.02284000 & 1.17053200 \\
\hline $\mathrm{H}$ & 1.98816900 & 14.57030400 & 0.27921100 \\
\hline $\mathrm{C}$ & 3.29444300 & 13.04175300 & 1.07393100 \\
\hline $\mathrm{C}$ & 3.73259800 & 12.31563900 & 2.22125800 \\
\hline $\mathrm{C}$ & 3.91401400 & 12.78411700 & -0.16664900 \\
\hline $\mathrm{H}$ & 3.60093700 & 13.34338600 & -1.04313400 \\
\hline $\mathrm{C}$ & 4.91890700 & 11.86102000 & -0.25338200 \\
\hline $\mathrm{H}$ & 5.42752300 & 11.69089700 & -1.19331500 \\
\hline $\mathrm{C}$ & 5.34418000 & 11.21813400 & 0.92300600 \\
\hline $\mathrm{C}$ & 6.51246900 & 10.29954800 & 0.88795200 \\
\hline $\mathrm{C}$ & 6.97009700 & 9.68424900 & -0.27578900 \\
\hline $\mathrm{H}$ & 6.47509300 & 9.84892500 & -1.22467500 \\
\hline $\mathrm{C}$ & 8.07968500 & 8.84912800 & -0.20743500 \\
\hline
\end{tabular}




\begin{tabular}{|c|c|c|c|}
\hline $\mathrm{H}$ & 8.46285000 & 8.37738400 & -1.10590400 \\
\hline $\mathrm{C}$ & 8.69993700 & 8.63997500 & 1.02000700 \\
\hline $\mathrm{H}$ & 9.57638800 & 8.00957700 & 1.11038800 \\
\hline $\mathrm{C}$ & 8.17923600 & 9.27887900 & 2.13574100 \\
\hline $\mathrm{H}$ & 8.61813000 & 9.14844200 & 3.11917700 \\
\hline $\mathrm{O}$ & 5.59027100 & 9.07494900 & 3.94953400 \\
\hline $\mathrm{O}$ & 3.54540900 & 5.47684600 & 4.14432600 \\
\hline $\mathrm{H}$ & 3.98367500 & 4.62062000 & 4.12216900 \\
\hline $\mathrm{H}$ & 2.73094000 & 5.37368600 & 3.64230300 \\
\hline $\mathrm{Fe}$ & 4.65677900 & 7.61369200 & 4.00360400 \\
\hline $\mathrm{N}$ & 5.13392800 & 7.06946500 & 6.02338200 \\
\hline $\mathrm{N}$ & 2.81040700 & 8.03126000 & 4.99218300 \\
\hline $\mathrm{N}$ & 3.56780200 & 7.86353000 & 2.14324200 \\
\hline $\mathrm{N}$ & 5.80845000 & 6.42748500 & 2.62914100 \\
\hline $\mathrm{C}$ & 6.30867400 & 6.73510100 & 6.53813400 \\
\hline $\mathrm{H}$ & 7.16452900 & 6.79173400 & 5.87234500 \\
\hline $\mathrm{C}$ & 6.46252500 & 6.33448800 & 7.87173800 \\
\hline $\mathrm{H}$ & 7.44200500 & 6.05591900 & 8.24188900 \\
\hline $\mathrm{C}$ & 5.34937500 & 6.28728300 & 8.68260900 \\
\hline $\mathrm{H}$ & 5.43015600 & 5.95783700 & 9.71415400 \\
\hline $\mathrm{C}$ & 4.09450500 & 6.67871400 & 8.17053400 \\
\hline $\mathrm{C}$ & 4.04189800 & 7.08355700 & 6.82453000 \\
\hline $\mathrm{C}$ & 2.89492900 & 6.68145800 & 8.95947100 \\
\hline $\mathrm{H}$ & 2.94777800 & 6.34883900 & 9.99099300 \\
\hline $\mathrm{C}$ & 1.71493400 & 7.07667300 & 8.42000400 \\
\hline $\mathrm{H}$ & 0.80397000 & 7.05923200 & 9.00956100 \\
\hline $\mathrm{C}$ & 1.64382200 & 7.52588600 & 7.05838500 \\
\hline $\mathrm{C}$ & 2.80050400 & 7.55416100 & 6.26509500 \\
\hline $\mathrm{C}$ & 0.44277500 & 7.92840300 & 6.43899300 \\
\hline $\mathrm{H}$ & -0.49567000 & 7.85937100 & 6.98095500 \\
\hline $\mathrm{C}$ & 0.46257300 & 8.38507000 & 5.14557000 \\
\hline $\mathrm{H}$ & -0.46683100 & 8.64666200 & 4.65659800 \\
\hline $\mathrm{C}$ & 1.68765100 & 8.48433700 & 4.43890500 \\
\hline $\mathrm{C}$ & 1.68989000 & 9.11876100 & 3.09168300 \\
\hline $\mathrm{C}$ & 0.74095000 & 10.10381400 & 2.86929000 \\
\hline $\mathrm{H}$ & 0.11312900 & 10.42629400 & 3.69172100 \\
\hline $\mathrm{C}$ & 0.53792500 & 10.71907000 & 1.62126100 \\
\hline $\mathrm{H}$ & -0.22217900 & 11.48451000 & 1.51832400 \\
\hline $\mathrm{C}$ & 1.28409900 & 10.31893500 & 0.54857000 \\
\hline $\mathrm{H}$ & 1.12180200 & 10.74317600 & -0.43704900 \\
\hline $\mathrm{C}$ & 2.28498500 & 9.33236200 & 0.71680100 \\
\hline $\mathrm{C}$ & 2.54035800 & 8.75481200 & 1.99446500 \\
\hline $\mathrm{C}$ & 3.05277300 & 8.89353500 & -0.38380400 \\
\hline $\mathrm{H}$ & 2.85437500 & 9.30910200 & -1.36694700 \\
\hline $\mathrm{C}$ & 4.03125300 & 7.95470300 & -0.21001500 \\
\hline $\mathrm{H}$ & 4.62401000 & 7.61931900 & -1.05036200 \\
\hline
\end{tabular}




$\begin{array}{lccc}\mathrm{C} & 4.28302600 & 7.47584000 & 1.09054100 \\ \mathrm{C} & 5.41015600 & 6.53746700 & 1.34879300 \\ \mathrm{C} & 6.02728200 & 5.80262700 & 0.33919200 \\ \mathrm{H} & 5.67835700 & 5.85809200 & -0.68429100 \\ \mathrm{C} & 7.08334300 & 4.95591300 & 0.66129400 \\ \mathrm{H} & 7.56663700 & 4.36825200 & -0.11184200 \\ \mathrm{C} & 7.49761200 & 4.86286800 & 1.98462400 \\ \mathrm{H} & 8.31125800 & 4.21057800 & 2.27921700 \\ \mathrm{C} & 6.82423500 & 5.61528500 & 2.93737600 \\ \mathrm{H} & 7.09502900 & 5.55619400 & 3.98636900\end{array}$

$\begin{array}{lrrr}\text { Complex 1 } & & & \\ 35 & & & \\ \mathrm{Fe} & -0.45779600 & 0.40710200 & 1.66117600 \\ \mathrm{Cl} & -1.05106700 & 0.14399100 & 3.81770200 \\ \mathrm{~N} & 1.25597100 & 1.14409000 & 2.41868200 \\ \mathrm{C} & 1.50688800 & 2.25971200 & 3.08819100 \\ \mathrm{H} & 0.66032600 & 2.88904200 & 3.33385900 \\ \mathrm{~N} & 0.56496500 & -1.25936400 & 1.63069300 \\ \mathrm{C} & 2.80010700 & 2.60028800 & 3.51424700 \\ \mathrm{H} & 2.94951300 & 3.52447700 & 4.05975000 \\ \mathrm{~N} & -2.18292200 & -0.47707000 & 1.27427800 \\ \mathrm{C} & 3.84955600 & 1.74618400 & 3.25462300 \\ \mathrm{H} & 4.85678700 & 1.99237400 & 3.57654300 \\ \mathrm{~N} & -1.55583000 & 2.05786100 & 1.49026400 \\ \mathrm{C} & 3.59245600 & 0.51401600 & 2.61694000 \\ \mathrm{C} & 2.26519800 & 0.25726500 & 2.24414000 \\ \mathrm{C} & 4.58554800 & -0.49482800 & 2.37228800 \\ \mathrm{H} & 5.61456300 & -0.28874900 & 2.64823800 \\ \mathrm{C} & 4.24364100 & -1.69635500 & 1.83643200 \\ \mathrm{H} & 4.99670200 & -2.45842900 & 1.66638000 \\ \mathrm{C} & 2.87348800 & -2.00405600 & 1.54279000 \\ \mathrm{C} & 1.89535300 & -1.03052100 & 1.76210500 \\ \mathrm{C} & 2.41067200 & -3.24330300 & 1.05416400 \\ \mathrm{H} & 3.11760900 & -4.02706200 & 0.80062500 \\ \mathrm{C} & 1.06258200 & -3.45961600 & 0.92529800 \\ \mathrm{H} & 0.71607800 & -4.41039300 & 0.54442500 \\ \mathrm{C} & 0.11968000 & -2.46308200 & 1.29410900 \\ \mathrm{C} & -1.32403600 & -2.78485100 & 1.36048600 \\ \mathrm{C} & -1.65234900 & -4.11928100 & 1.54591000 \\ \mathrm{H} & -0.86195600 & -4.84750400 & 1.67854900 \\ \mathrm{C} & -2.97309700 & -4.59192300 & 1.62447000 \\ \mathrm{H} & -3.15751200 & -5.64840100 & 1.78066300 \\ \mathrm{C} & -4.00403200 & -3.70255500 & 1.52458400 \\ \mathrm{H} & -5.03661700 & -4.03099000 & 1.58550600\end{array}$




\begin{tabular}{|c|c|c|c|}
\hline $\mathrm{C}$ & -3.73249100 & -2.31975300 & 1.38861900 \\
\hline $\mathrm{C}$ & -2.39326500 & -1.83088700 & 1.32258700 \\
\hline $\mathrm{C}$ & -4.80067900 & -1.39988100 & 1.36560400 \\
\hline $\mathrm{H}$ & -5.81985300 & -1.76815200 & 1.43069100 \\
\hline $\mathrm{C}$ & -4.54895500 & -0.05865200 & 1.30581700 \\
\hline $\mathrm{H}$ & -5.36153900 & 0.65458400 & 1.33461200 \\
\hline $\mathrm{C}$ & -3.21346300 & 0.37097700 & 1.28533300 \\
\hline $\mathrm{C}$ & -2.86265000 & 1.80370600 & 1.30622400 \\
\hline $\mathrm{C}$ & -3.77603100 & 2.83540400 & 1.10854500 \\
\hline $\mathrm{H}$ & -4.83032600 & 2.62850100 & 0.97713600 \\
\hline $\mathrm{C}$ & -3.32097000 & 4.14823700 & 1.08341300 \\
\hline $\mathrm{H}$ & -4.02081100 & 4.96691500 & 0.95544600 \\
\hline $\mathrm{C}$ & -1.95797600 & 4.39103000 & 1.20794100 \\
\hline $\mathrm{H}$ & -1.55565600 & 5.39604000 & 1.16612200 \\
\hline $\mathrm{C}$ & -1.10796000 & 3.31201400 & 1.39783000 \\
\hline $\mathrm{H}$ & -0.03730100 & 3.44848400 & 1.46648800 \\
\hline $\mathrm{O}$ & 0.01264800 & 0.66857800 & 0.09848600 \\
\hline $\mathrm{O}$ & 0.96615400 & 0.84944300 & -3.39360200 \\
\hline $\mathrm{Fe}$ & 0.53451200 & 0.74041500 & -1.84311600 \\
\hline $\mathrm{N}$ & 2.38687000 & 1.15124500 & -1.13113900 \\
\hline $\mathrm{N}$ & 1.23167200 & -1.10170100 & -1.84395600 \\
\hline $\mathrm{N}$ & -1.33157800 & 0.19247700 & -2.13774600 \\
\hline $\mathrm{N}$ & -0.23289900 & 2.57332700 & -2.01270900 \\
\hline $\mathrm{C}$ & 2.89662600 & 2.23596500 & -0.56649500 \\
\hline $\mathrm{H}$ & 2.20451400 & 3.03325300 & -0.32388400 \\
\hline $\mathrm{C}$ & 4.26511800 & 2.36635400 & -0.28985000 \\
\hline $\mathrm{H}$ & 4.63281000 & 3.28557400 & 0.15023000 \\
\hline $\mathrm{C}$ & 5.12227600 & 1.33519000 & -0.61062200 \\
\hline $\mathrm{H}$ & 6.19089900 & 1.43004500 & -0.44409100 \\
\hline $\mathrm{C}$ & 4.59541500 & 0.14146700 & -1.14553900 \\
\hline $\mathrm{C}$ & 3.20954100 & 0.09824200 & -1.35685200 \\
\hline $\mathrm{C}$ & 5.38097100 & -1.01634900 & -1.47302500 \\
\hline $\mathrm{H}$ & 6.45705300 & -0.96980500 & -1.34212400 \\
\hline $\mathrm{C}$ & 4.79435300 & -2.14318500 & -1.95619700 \\
\hline $\mathrm{H}$ & 5.39726700 & -3.00422000 & -2.22568900 \\
\hline $\mathrm{C}$ & 3.36948300 & -2.22035400 & -2.11879400 \\
\hline $\mathrm{C}$ & 2.58740800 & -1.10836300 & -1.79598900 \\
\hline $\mathrm{C}$ & 2.67320200 & -3.34214000 & -2.61590400 \\
\hline $\mathrm{H}$ & 3.22039200 & -4.21064400 & -2.96953000 \\
\hline $\mathrm{C}$ & 1.30191400 & -3.32128100 & -2.66902800 \\
\hline $\mathrm{H}$ & 0.77964000 & -4.16657600 & -3.09630600 \\
\hline $\mathrm{C}$ & 0.57062100 & -2.19157800 & -2.21753200 \\
\hline $\mathrm{C}$ & -0.90955600 & -2.23717700 & -2.13114300 \\
\hline $\mathrm{C}$ & -1.48549600 & -3.49271200 & -2.01762900 \\
\hline $\mathrm{H}$ & -0.84564100 & -4.36383200 & -1.94978700 \\
\hline $\mathrm{C}$ & -2.87219700 & -3.71512500 & -1.9754220 \\
\hline
\end{tabular}




$\begin{array}{lrrr}\mathrm{H} & -3.25217000 & -4.72740100 & -1.90478200 \\ \mathrm{C} & -3.71855000 & -2.64623800 & -2.04441900 \\ \mathrm{H} & -4.79532800 & -2.78154100 & -2.04532400 \\ \mathrm{C} & -3.19344200 & -1.33325100 & -2.10840200 \\ \mathrm{C} & -1.78569400 & -1.09932300 & -2.12844400 \\ \mathrm{C} & -4.07170500 & -0.23020200 & -2.13959500 \\ \mathrm{H} & -5.14268500 & -0.40675700 & -2.14866800 \\ \mathrm{C} & -3.57612900 & 1.04256200 & -2.15797700 \\ \mathrm{H} & -4.24374100 & 1.89302800 & -2.17896600 \\ \mathrm{C} & -2.18351000 & 1.21911500 & -2.14304200 \\ \mathrm{C} & -1.57171300 & 2.56391100 & -2.15672400 \\ \mathrm{C} & -2.28085800 & 3.73591800 & -2.39420400 \\ \mathrm{H} & -3.35479400 & 3.72267000 & -2.52727400 \\ \mathrm{C} & -1.58705100 & 4.93568600 & -2.51837600 \\ \mathrm{H} & -2.12107000 & 5.85742900 & -2.72237700 \\ \mathrm{C} & -0.20137300 & 4.92306800 & -2.43281800 \\ \mathrm{H} & 0.38379300 & 5.82294200 & -2.58063000 \\ \mathrm{C} & 0.43820200 & 3.71434100 & -2.19153400 \\ \mathrm{H} & 1.51799400 & 3.64858500 & -2.18679900\end{array}$

\begin{tabular}{|c|c|c|c|}
\hline \multicolumn{2}{|c|}{$\begin{array}{l}\text { Nitrate attack patway } \\
\text { TS11, }\end{array}$} & & \\
\hline $\mathrm{Fe}$ & 0.26986300 & -0.19778500 & -2.05999200 \\
\hline $\mathrm{Cl}$ & 0.71993200 & -1.04218400 & -4.14608700 \\
\hline $\mathrm{N}$ & -1.56450300 & 0.21349200 & -2.79130000 \\
\hline $\mathrm{C}$ & -1.96864900 & 1.08029500 & -3.70474400 \\
\hline $\mathrm{H}$ & -1.19520500 & 1.63134800 & -4.22702700 \\
\hline $\mathrm{N}$ & -0.63103700 & -1.81611900 & -1.41549000 \\
\hline $\mathrm{C}$ & -3.32518300 & 1.25501100 & -4.02290800 \\
\hline $\mathrm{H}$ & -3.60288200 & 1.97824700 & -4.78026600 \\
\hline $\mathrm{N}$ & 2.07872200 & -0.86031800 & -1.58904600 \\
\hline $\mathrm{C}$ & -4.27562100 & 0.49294600 & -3.38004600 \\
\hline $\mathrm{H}$ & -5.33057400 & 0.61425000 & -3.60606100 \\
\hline $\mathrm{N}$ & 1.26819200 & 1.50005400 & -2.41034900 \\
\hline $\mathrm{C}$ & -3.86203200 & -0.48852800 & -2.45588300 \\
\hline $\mathrm{C}$ & -2.48413800 & -0.59815100 & -2.22127500 \\
\hline $\mathrm{C}$ & -4.75197700 & -1.38949200 & -1.77643900 \\
\hline $\mathrm{H}$ & -5.81978200 & -1.28604300 & -1.94009900 \\
\hline $\mathrm{C}$ & -4.27167300 & -2.36715600 & -0.96475500 \\
\hline $\mathrm{H}$ & -4.94997900 & -3.04884300 & -0.46262300 \\
\hline $\mathrm{C}$ & -2.85847900 & -2.55216700 & -0.79546500 \\
\hline $\mathrm{C}$ & -1.97565600 & -1.67160600 & -1.42733700 \\
\hline $\mathrm{C}$ & -2.26598800 & -3.58510700 & -0.04173100 \\
\hline $\mathrm{H}$ & -2.88733000 & -4.27011700 & 0.52650900 \\
\hline $\mathrm{C}$ & -0.90239200 & -3.72872100 & -0.04843300 \\
\hline
\end{tabular}




\begin{tabular}{|c|c|c|c|}
\hline $\mathrm{H}$ & -0.45090200 & -4.51794400 & 0.53731200 \\
\hline $\mathrm{C}$ & -0.07946100 & -2.85456300 & -0.80829700 \\
\hline $\mathrm{C}$ & 1.37156900 & -3.13040600 & -0.95759500 \\
\hline $\mathrm{C}$ & 1.77040200 & -4.44726100 & -0.80096400 \\
\hline $\mathrm{H}$ & 1.02062400 & -5.21234300 & -0.64175200 \\
\hline $\mathrm{C}$ & 3.10923800 & -4.86794900 & -0.87813500 \\
\hline $\mathrm{H}$ & 3.34999700 & -5.91717500 & -0.75248400 \\
\hline $\mathrm{C}$ & 4.08521700 & -3.94592500 & -1.12496700 \\
\hline $\mathrm{H}$ & 5.12905600 & -4.23577300 & -1.18995200 \\
\hline $\mathrm{C}$ & 3.73555100 & -2.59304100 & -1.34639800 \\
\hline $\mathrm{C}$ & 2.37775000 & -2.16261200 & -1.29120700 \\
\hline $\mathrm{C}$ & 4.73750800 & -1.65254200 & -1.66940000 \\
\hline $\mathrm{H}$ & 5.77085400 & -1.97985200 & -1.72860100 \\
\hline $\mathrm{C}$ & 4.40082000 & -0.35803500 & -1.93740200 \\
\hline $\mathrm{H}$ & 5.15650400 & 0.36042300 & -2.22547400 \\
\hline $\mathrm{C}$ & 3.04146100 & 0.00127100 & -1.90285600 \\
\hline $\mathrm{C}$ & 2.59256200 & 1.36913500 & -2.23737500 \\
\hline $\mathrm{C}$ & 3.44811600 & 2.46575300 & -2.30807800 \\
\hline $\mathrm{H}$ & 4.51517700 & 2.34921800 & -2.16733900 \\
\hline $\mathrm{C}$ & 2.91384800 & 3.72459000 & -2.55127200 \\
\hline $\mathrm{H}$ & 3.56321900 & 4.59014700 & -2.62287200 \\
\hline $\mathrm{C}$ & 1.53733400 & 3.85591500 & -2.68366900 \\
\hline $\mathrm{H}$ & 1.07438700 & 4.82095900 & -2.85028500 \\
\hline $\mathrm{C}$ & 0.75315300 & 2.71446800 & -2.59521200 \\
\hline $\mathrm{H}$ & -0.32576400 & 2.77068800 & -2.65805700 \\
\hline $\mathrm{O}$ & -0.11630100 & 0.53596400 & -0.51179200 \\
\hline $\mathrm{O}$ & -0.90556600 & 1.77079000 & 2.64892200 \\
\hline $\mathrm{Fe}$ & -0.52025200 & 1.14028000 & 1.15831300 \\
\hline $\mathrm{N}$ & -2.43780700 & 1.38357400 & 0.53806400 \\
\hline $\mathrm{N}$ & -1.23807800 & -0.56344300 & 1.83908000 \\
\hline $\mathrm{N}$ & 1.35498500 & 0.68054000 & 1.57174500 \\
\hline $\mathrm{N}$ & 0.27795100 & 2.95650300 & 0.80765700 \\
\hline $\mathrm{C}$ & -2.98683800 & 2.25101500 & -0.29348600 \\
\hline $\mathrm{H}$ & -2.30765900 & 2.89148300 & -0.84423300 \\
\hline $\mathrm{C}$ & -4.37635100 & 2.34994500 & -0.46454800 \\
\hline $\mathrm{H}$ & -4.77685100 & 3.09210200 & -1.14456300 \\
\hline $\mathrm{C}$ & -5.20675300 & 1.51492300 & 0.25107500 \\
\hline $\mathrm{H}$ & -6.28570200 & 1.59554900 & 0.16227400 \\
\hline $\mathrm{C}$ & -4.64143500 & 0.53419500 & 1.09099100 \\
\hline $\mathrm{C}$ & -3.24166800 & 0.49984500 & 1.17198900 \\
\hline $\mathrm{C}$ & -5.40003200 & -0.42388700 & 1.84681000 \\
\hline $\mathrm{H}$ & -6.48334500 & -0.37692800 & 1.80813900 \\
\hline $\mathrm{C}$ & -4.77805800 & -1.36429400 & 2.60472900 \\
\hline $\mathrm{H}$ & -5.35857700 & -2.07251500 & 3.18696800 \\
\hline $\mathrm{C}$ & -3.34492200 & -1.44837300 & 2.64904000 \\
\hline $\mathrm{C}$ & -2.58897200 & -0.52831600 & 1.9193230 \\
\hline
\end{tabular}




\begin{tabular}{lrrr}
$\mathrm{C}$ & -2.61382600 & -2.38854100 & 3.40464000 \\
$\mathrm{H}$ & -3.13246200 & -3.08151100 & 4.05979000 \\
$\mathrm{C}$ & -1.24501500 & -2.40835100 & 3.32162800 \\
$\mathrm{H}$ & -0.68672300 & -3.09803900 & 3.94005600 \\
$\mathrm{C}$ & -0.55373500 & -1.50427200 & 2.47270400 \\
$\mathrm{C}$ & 0.91032400 & -1.63384300 & 2.27079500 \\
$\mathrm{C}$ & 1.45683400 & -2.88851400 & 2.47387000 \\
$\mathrm{H}$ & 0.80161500 & -3.71581700 & 2.71812500 \\
$\mathrm{C}$ & 2.83057100 & -3.16634700 & 2.36616700 \\
$\mathrm{H}$ & 3.19051800 & -4.17277400 & 2.54345300 \\
$\mathrm{C}$ & 3.69171600 & -2.15484100 & 2.05444200 \\
$\mathrm{H}$ & 4.76109400 & -2.32698800 & 1.98957200 \\
$\mathrm{C}$ & 3.19215400 & -0.85525100 & 1.80295400 \\
$\mathrm{C}$ & 1.79676500 & -0.57633700 & 1.87400900 \\
$\mathrm{C}$ & 4.08029100 & 0.18865100 & 1.46974400 \\
$\mathrm{H}$ & 5.14514300 & -0.01646200 & 1.42933400 \\
$\mathrm{C}$ & 3.59967400 & 1.43759500 & 1.20268400 \\
$\mathrm{H}$ & 4.27003000 & 2.24481100 & 0.94032400 \\
$\mathrm{C}$ & 2.21082500 & 1.64579500 & 1.25050600 \\
$\mathrm{C}$ & 1.61253800 & 2.96261000 & 0.95360300 \\
$\mathrm{C}$ & 2.34180000 & 4.14639700 & 0.91132500 \\
$\mathrm{H}$ & 3.41331900 & 4.14536000 & 1.06373900 \\
$\mathrm{C}$ & 1.66918800 & 5.34811100 & 0.72674100 \\
$\mathrm{H}$ & 2.21698200 & 6.28374500 & 0.70578200 \\
$\mathrm{C}$ & 0.28498400 & 5.33373400 & 0.61846400 \\
$\mathrm{H}$ & -0.28494600 & 6.24971400 & 0.51945000 \\
$\mathrm{C}$ & -0.37261400 & 4.11292400 & 0.68181200 \\
$\mathrm{H}$ & -1.45322400 & 4.06082200 & 0.67561100 \\
$\mathrm{O}$ & 0.17007900 & 0.63049700 & 4.48516800 \\
$\mathrm{~N}$ & 1.31282600 & 1.22757400 & 4.50764300 \\
$\mathrm{O}$ & 2.22332500 & 0.48765100 & 4.85873900 \\
$\mathrm{O}$ & 1.40240200 & 2.38826400 & 4.18838100 \\
& & & \\
\hline & & & \\
$\mathrm{H}$ & & &
\end{tabular}

$\begin{array}{lccc}\text { Int1 }{ }^{\prime}{ }^{2} & & & \\ 23 & & & \\ \mathrm{Fe} & 10.33837800 & 7.03714000 & 2.29927900 \\ \mathrm{Cl} & 12.14401200 & 8.41592500 & 2.02771700 \\ \mathrm{~N} & 11.30881700 & 6.33473200 & 3.92432100 \\ \mathrm{C} & 12.41473100 & 5.61955700 & 4.04363400 \\ \mathrm{H} & 12.97145800 & 5.43059100 & 3.13295800 \\ \mathrm{~N} & 9.45361000 & 8.16678200 & 3.63712100 \\ \mathrm{C} & 12.87689400 & 5.15719200 & 5.28662400 \\ \mathrm{H} & 13.78965600 & 4.57509600 & 5.32928300 \\ \mathrm{~N} & 9.56803700 & 8.02240100 & 0.76112800 \\ \mathrm{C} & 12.17105600 & 5.46379600 & 6.42902700 \\ \mathrm{H} & 12.50549500 & 5.11125100 & 7.39984600\end{array}$




\begin{tabular}{|c|c|c|c|}
\hline $\mathrm{N}$ & 10.93508900 & 5.78701700 & 0.86026300 \\
\hline $\mathrm{C}$ & 11.02944000 & 6.28624300 & 6.33361600 \\
\hline $\mathrm{C}$ & 10.66204600 & 6.71376700 & 5.05014400 \\
\hline $\mathrm{C}$ & 10.25181200 & 6.72987200 & 7.45782300 \\
\hline $\mathrm{H}$ & 10.52233400 & 6.37685200 & 8.44769300 \\
\hline $\mathrm{C}$ & 9.21366100 & 7.59069700 & 7.29425900 \\
\hline $\mathrm{H}$ & 8.63608100 & 7.92586000 & 8.14926000 \\
\hline $\mathrm{C}$ & 8.88884300 & 8.11088400 & 5.99662100 \\
\hline $\mathrm{C}$ & 9.61653300 & 7.67343200 & 4.88614700 \\
\hline $\mathrm{C}$ & 7.88096200 & 9.06146900 & 5.73851300 \\
\hline $\mathrm{H}$ & 7.23609000 & 9.40322300 & 6.54192800 \\
\hline $\mathrm{C}$ & 7.73286900 & 9.56164600 & 4.47028200 \\
\hline $\mathrm{H}$ & 6.95063200 & 10.28245300 & 4.27488600 \\
\hline $\mathrm{C}$ & 8.58463300 & 9.14024400 & 3.41422800 \\
\hline $\mathrm{C}$ & 8.53227300 & 9.81432300 & 2.09253500 \\
\hline $\mathrm{C}$ & 8.05797400 & 11.11534600 & 2.08087100 \\
\hline $\mathrm{H}$ & 7.80849200 & 11.59625600 & 3.01862700 \\
\hline $\mathrm{C}$ & 7.91609700 & 11.88040500 & 0.91048000 \\
\hline $\mathrm{H}$ & 7.54397700 & 12.89624200 & 0.97535000 \\
\hline $\mathrm{C}$ & 8.26540500 & 11.33339500 & -0.29039600 \\
\hline $\mathrm{H}$ & 8.16904500 & 11.89564900 & -1.21365300 \\
\hline $\mathrm{C}$ & 8.80652300 & 10.02700200 & -0.33822200 \\
\hline $\mathrm{C}$ & 8.97093200 & 9.25159800 & 0.84669300 \\
\hline $\mathrm{C}$ & 9.23284800 & 9.48898400 & -1.57165600 \\
\hline $\mathrm{H}$ & 9.12458900 & 10.08327300 & -2.47361700 \\
\hline $\mathrm{C}$ & 9.80517800 & 8.25151500 & -1.61790400 \\
\hline $\mathrm{H}$ & 10.17115300 & 7.84720000 & -2.55215100 \\
\hline $\mathrm{C}$ & 9.97232000 & 7.54621000 & -0.41299300 \\
\hline $\mathrm{C}$ & 10.63237300 & 6.22447700 & -0.37219000 \\
\hline $\mathrm{C}$ & 10.87540300 & 5.44505100 & -1.5002210 \\
\hline $\mathrm{H}$ & 10.63092600 & 5.80738600 & -2.4906650 \\
\hline $\mathrm{C}$ & 11.43002500 & 4.18138500 & -1.3411790 \\
\hline $\mathrm{H}$ & 11.63953200 & 3.56320600 & -2.2071720 \\
\hline $\mathrm{C}$ & 11.69372100 & 3.71905900 & -0.05800000 \\
\hline $\mathrm{H}$ & 12.10184300 & 2.73067800 & 0.11470800 \\
\hline $\mathrm{C}$ & 11.41995900 & 4.55549400 & 1.01476000 \\
\hline $\mathrm{H}$ & 11.57909900 & 4.23020900 & 2.03469500 \\
\hline $\mathrm{O}$ & 9.02681200 & 5.89412500 & 2.54151700 \\
\hline $\mathrm{O}$ & 6.15648900 & 3.69373900 & 3.15124200 \\
\hline $\mathrm{Fe}$ & 7.59415800 & 4.87687900 & 2.78989800 \\
\hline $\mathrm{N}$ & 8.39118000 & 4.22338000 & 4.53364600 \\
\hline $\mathrm{N}$ & 6.60464100 & 6.08850700 & 3.97482200 \\
\hline $\mathrm{N}$ & 6.87717300 & 5.68690400 & 1.13517200 \\
\hline $\mathrm{N}$ & 8.17633400 & 3.44352600 & 1.52835900 \\
\hline $\mathrm{C}$ & 9.42829100 & 3.44731100 & 4.79853300 \\
\hline $\mathrm{H}$ & 10.03980400 & 3.15142100 & 3.9534220 \\
\hline
\end{tabular}




\begin{tabular}{|c|c|c|c|}
\hline $\mathrm{C}$ & 9.73399900 & 3.01417700 & 6.09757300 \\
\hline $\mathrm{H}$ & 10.58528600 & 2.36301700 & 6.25555300 \\
\hline $\mathrm{C}$ & 8.93038600 & 3.40699500 & 7.14570700 \\
\hline $\mathrm{H}$ & 9.12345300 & 3.05725300 & 8.15516700 \\
\hline $\mathrm{C}$ & 7.85771300 & 4.28732800 & 6.90013100 \\
\hline $\mathrm{C}$ & 7.65452000 & 4.68616200 & 5.57034400 \\
\hline $\mathrm{C}$ & 6.98345400 & 4.79921500 & 7.91930600 \\
\hline $\mathrm{H}$ & 7.12407800 & 4.46421300 & 8.94171300 \\
\hline $\mathrm{C}$ & 5.99154200 & 5.67382000 & 7.61109600 \\
\hline $\mathrm{H}$ & 5.32187900 & 6.04034900 & 8.38231200 \\
\hline $\mathrm{C}$ & 5.80859200 & 6.13713900 & 6.26385700 \\
\hline $\mathrm{C}$ & 6.65301900 & 5.65939800 & 5.25864100 \\
\hline $\mathrm{C}$ & 4.80343700 & 7.03699100 & 5.85406300 \\
\hline $\mathrm{H}$ & 4.06345600 & 7.38636800 & 6.56741800 \\
\hline $\mathrm{C}$ & 4.75102900 & 7.44823400 & 4.54646800 \\
\hline $\mathrm{H}$ & 3.94582300 & 8.09529200 & 4.22571300 \\
\hline $\mathrm{C}$ & 5.71039900 & 6.99566100 & 3.60468000 \\
\hline $\mathrm{C}$ & 5.73276300 & 7.55894000 & 2.23107500 \\
\hline $\mathrm{C}$ & 5.21130600 & 8.83022000 & 2.06854300 \\
\hline $\mathrm{H}$ & 4.85883800 & 9.37149500 & 2.93833500 \\
\hline $\mathrm{C}$ & 5.12427400 & 9.48162300 & 0.82521200 \\
\hline $\mathrm{H}$ & 4.70068500 & 10.47742700 & 0.77009000 \\
\hline $\mathrm{C}$ & 5.56257600 & 8.84077900 & -0.29755400 \\
\hline $\mathrm{H}$ & 5.48666800 & 9.30526900 & -1.27533400 \\
\hline $\mathrm{C}$ & 6.13681000 & 7.55160400 & -0.19426800 \\
\hline $\mathrm{C}$ & 6.26034000 & 6.90184100 & 1.06771300 \\
\hline $\mathrm{C}$ & 6.61438700 & 6.89189200 & -1.34712000 \\
\hline $\mathrm{H}$ & 6.51022500 & 7.37511600 & -2.31332800 \\
\hline $\mathrm{C}$ & 7.20072700 & 5.66361900 & -1.24135700 \\
\hline $\mathrm{H}$ & 7.57505000 & 5.15213300 & -2.11796200 \\
\hline $\mathrm{C}$ & 7.32990800 & 5.09059800 & 0.03607200 \\
\hline $\mathrm{C}$ & 7.95837300 & 3.76817800 & 0.24228500 \\
\hline $\mathrm{C}$ & 8.20687800 & 2.86691100 & -0.78568800 \\
\hline $\mathrm{H}$ & 8.00586900 & 3.12822300 & -1.81652800 \\
\hline $\mathrm{C}$ & 8.65982400 & 1.59097700 & -0.47053400 \\
\hline $\mathrm{H}$ & 8.84254600 & 0.86622900 & -1.25623400 \\
\hline $\mathrm{C}$ & 8.82260200 & 1.24621500 & 0.86381100 \\
\hline $\mathrm{H}$ & 9.12266200 & 0.24788300 & 1.15843900 \\
\hline $\mathrm{C}$ & 8.55602100 & 2.20351000 & 1.83367800 \\
\hline $\mathrm{H}$ & 8.61020700 & 1.96088000 & 2.88667200 \\
\hline $\mathrm{O}$ & 4.93291700 & 4.22471600 & 2.77732300 \\
\hline $\mathrm{N}$ & 4.53787900 & 3.76419800 & 1.48473900 \\
\hline $\mathrm{O}$ & 3.51406700 & 4.28383300 & 1.13491100 \\
\hline $\mathrm{O}$ & 5.24053300 & 2.96506100 & 0.92963600 \\
\hline
\end{tabular}

Water attack pathway 


\begin{tabular}{|c|c|c|c|}
\hline & & & \\
\hline $\mathrm{Fe}$ & 10.28672300 & 6.87658400 & 2.28323300 \\
\hline $\mathrm{Cl}$ & 12.06417300 & 8.19030500 & 2.03851100 \\
\hline $\mathrm{N}$ & 11.37300100 & 6.04891300 & 4.11513000 \\
\hline $\mathrm{C}$ & 12.41014700 & 5.25182600 & 4.29877000 \\
\hline $\mathrm{H}$ & 12.93070800 & 4.91652000 & 3.40659000 \\
\hline $\mathrm{N}$ & 9.51940600 & 8.03257700 & 3.72767900 \\
\hline $\mathrm{C}$ & 12.86581600 & 4.86740600 & 5.57079400 \\
\hline $\mathrm{H}$ & 13.73412100 & 4.22542000 & 5.66225800 \\
\hline $\mathrm{N}$ & 9.38082700 & 8.04301400 & 0.69922600 \\
\hline $\mathrm{C}$ & 12.19697900 & 5.33224000 & 6.67907200 \\
\hline $\mathrm{H}$ & 12.51670800 & 5.05453100 & 7.67896500 \\
\hline $\mathrm{N}$ & 10.82459200 & 5.80145600 & 0.66024800 \\
\hline $\mathrm{C}$ & 11.10236800 & 6.20763200 & 6.51364800 \\
\hline $\mathrm{C}$ & 10.74760900 & 6.56088200 & 5.19677200 \\
\hline $\mathrm{C}$ & 10.36536800 & 6.74162800 & 7.62274800 \\
\hline $\mathrm{H}$ & 10.65193200 & 6.45028400 & 8.62825300 \\
\hline $\mathrm{C}$ & 9.34149000 & 7.60592900 & 7.41860400 \\
\hline $\mathrm{H}$ & 8.78342300 & 8.01405500 & 8.25476400 \\
\hline $\mathrm{C}$ & 9.01322500 & 8.04766800 & 6.09475000 \\
\hline $\mathrm{C}$ & 9.72007300 & 7.55605000 & 4.98492000 \\
\hline $\mathrm{C}$ & 7.99645700 & 8.98404900 & 5.83169600 \\
\hline $\mathrm{H}$ & 7.37313700 & 9.34670300 & 6.64337600 \\
\hline $\mathrm{C}$ & 7.81786100 & 9.45492300 & 4.55775800 \\
\hline $\mathrm{H}$ & 7.03501700 & 10.17510000 & 4.36221700 \\
\hline $\mathrm{C}$ & 8.65920600 & 9.02766200 & 3.49853100 \\
\hline $\mathrm{C}$ & 8.58590200 & 9.77998100 & 2.20647700 \\
\hline $\mathrm{C}$ & 8.22688900 & 11.11508400 & 2.31173600 \\
\hline $\mathrm{H}$ & 8.09337500 & 11.55699200 & 3.29176000 \\
\hline $\mathrm{C}$ & 8.07004800 & 11.97000400 & 1.20297400 \\
\hline $\mathrm{H}$ & 7.79038200 & 13.00454100 & 1.36614200 \\
\hline $\mathrm{C}$ & 8.30076000 & 11.49745600 & -0.05793100 \\
\hline $\mathrm{H}$ & 8.20026800 & 12.14114700 & -0.92608000 \\
\hline $\mathrm{C}$ & 8.73800800 & 10.16262300 & -0.23020400 \\
\hline $\mathrm{C}$ & 8.89202900 & 9.29375700 & 0.88862500 \\
\hline $\mathrm{C}$ & 9.09018100 & 9.67456800 & -1.50911800 \\
\hline $\mathrm{H}$ & 8.98896500 & 10.32894400 & -2.36972300 \\
\hline $\mathrm{C}$ & 9.61149500 & 8.41704500 & -1.65273400 \\
\hline $\mathrm{H}$ & 9.94896300 & 8.07321600 & -2.62204400 \\
\hline $\mathrm{C}$ & 9.76639700 & 7.62832500 & -0.49557600 \\
\hline $\mathrm{C}$ & 10.44690300 & 6.30421300 & -0.5293760 \\
\hline $\mathrm{C}$ & 10.71514900 & 5.60913300 & -1.70658600 \\
\hline $\mathrm{H}$ & 10.41502500 & 6.01104800 & -2.66623800 \\
\hline $\mathrm{C}$ & 11.38998700 & 4.39536200 & -1.64509900 \\
\hline $\mathrm{H}$ & 11.62903100 & 3.85808600 & -2.5566720 \\
\hline
\end{tabular}




\begin{tabular}{|c|c|c|c|}
\hline $\mathrm{C}$ & 11.76058800 & 3.88616400 & -0.40527300 \\
\hline $\mathrm{H}$ & 12.29372300 & 2.94751300 & -0.31423200 \\
\hline $\mathrm{C}$ & 11.44820200 & 4.62392400 & 0.72654000 \\
\hline $\mathrm{H}$ & 11.70192800 & 4.27767200 & 1.72113600 \\
\hline $\mathrm{O}$ & 8.97055500 & 5.75911400 & 2.52988300 \\
\hline $\mathrm{O}$ & 6.15058700 & 3.78442700 & 3.01089400 \\
\hline $\mathrm{Fe}$ & 7.48297700 & 4.84575400 & 2.70644300 \\
\hline $\mathrm{N}$ & 8.24663700 & 4.22590800 & 4.48034900 \\
\hline $\mathrm{N}$ & 6.54260700 & 6.13493600 & 3.85207800 \\
\hline $\mathrm{N}$ & 6.85497300 & 5.66282100 & 1.02809100 \\
\hline $\mathrm{N}$ & 8.12239600 & 3.40363700 & 1.46804400 \\
\hline $\mathrm{C}$ & 9.28380000 & 3.45557600 & 4.76689900 \\
\hline $\mathrm{H}$ & 9.89334500 & 3.12599400 & 3.93451200 \\
\hline $\mathrm{C}$ & 9.60061900 & 3.08620300 & 6.08257700 \\
\hline $\mathrm{H}$ & 10.44860600 & 2.43653000 & 6.26161100 \\
\hline $\mathrm{C}$ & 8.82033400 & 3.54493800 & 7.12189700 \\
\hline $\mathrm{H}$ & 9.02656400 & 3.24532000 & 8.14470500 \\
\hline $\mathrm{C}$ & 7.76207700 & 4.43582000 & 6.84823600 \\
\hline $\mathrm{C}$ & 7.54199700 & 4.76422000 & 5.50284900 \\
\hline $\mathrm{C}$ & 6.92141900 & 5.02935500 & 7.85139900 \\
\hline $\mathrm{H}$ & 7.07047200 & 4.74797000 & 8.88859700 \\
\hline $\mathrm{C}$ & 5.95336800 & 5.92007600 & 7.51196800 \\
\hline $\mathrm{H}$ & 5.31170900 & 6.35209500 & 8.27292200 \\
\hline $\mathrm{C}$ & 5.77066500 & 6.32295600 & 6.14557400 \\
\hline $\mathrm{C}$ & 6.57867100 & 5.75771900 & 5.15484600 \\
\hline $\mathrm{C}$ & 4.81013500 & 7.25624900 & 5.70317100 \\
\hline $\mathrm{H}$ & 4.09565100 & 7.67656500 & 6.40444000 \\
\hline $\mathrm{C}$ & 4.77846600 & 7.62299300 & 4.38112000 \\
\hline $\mathrm{H}$ & 4.01579700 & 8.31104600 & 4.04214800 \\
\hline $\mathrm{C}$ & 5.70907900 & 7.08817300 & 3.45239000 \\
\hline $\mathrm{C}$ & 5.76391300 & 7.60823600 & 2.06675800 \\
\hline $\mathrm{C}$ & 5.30310800 & 8.89988700 & 1.86669200 \\
\hline $\mathrm{H}$ & 4.99056100 & 9.48801800 & 2.72087800 \\
\hline $\mathrm{C}$ & 5.22529800 & 9.51170000 & 0.60444300 \\
\hline $\mathrm{H}$ & 4.84917000 & 10.52420700 & 0.51969800 \\
\hline $\mathrm{C}$ & 5.61323800 & 8.80742300 & -0.49928500 \\
\hline $\mathrm{H}$ & 5.53853800 & 9.23578100 & -1.49349800 \\
\hline $\mathrm{C}$ & 6.14050400 & 7.50174500 & -0.35485300 \\
\hline $\mathrm{C}$ & 6.26277100 & 6.89254700 & 0.92921500 \\
\hline $\mathrm{C}$ & 6.57500000 & 6.78988400 & -1.49188900 \\
\hline $\mathrm{H}$ & 6.46430200 & 7.24057200 & -2.47304600 \\
\hline $\mathrm{C}$ & 7.13839400 & 5.55333800 & -1.35245800 \\
\hline $\mathrm{H}$ & 7.48579700 & 5.00714800 & -2.21860400 \\
\hline $\mathrm{C}$ & 7.28975600 & 5.02655300 & -0.05926100 \\
\hline $\mathrm{C}$ & 7.94160000 & 3.72132100 & 0.17399000 \\
\hline $\mathrm{C}$ & 8.29240900 & 2.83628000 & -0.8400000 \\
\hline
\end{tabular}




$\begin{array}{lrrr}\mathrm{H} & 8.14283600 & 3.09162200 & -1.88079800 \\ \mathrm{C} & 8.81432800 & 1.59167900 & -0.50460900 \\ \mathrm{H} & 9.09022400 & 0.88692000 & -1.28159000 \\ \mathrm{C} & 8.93928100 & 1.25324200 & 0.83655100 \\ \mathrm{H} & 9.30087500 & 0.27931600 & 1.14460900 \\ \mathrm{C} & 8.56897900 & 2.18909000 & 1.79316100 \\ \mathrm{H} & 8.60106100 & 1.94938700 & 2.84745300 \\ \mathrm{O} & 4.60782900 & 4.32781200 & 2.29859800 \\ \mathrm{H} & 4.57186700 & 3.66149700 & 1.54175600 \\ \mathrm{H} & 4.04812400 & 3.98580200 & 3.00976900 \\ \mathrm{O} & 4.61935600 & 2.66060500 & 0.31952400 \\ \mathrm{H} & 4.67992100 & 1.72555800 & 0.54198900 \\ \mathrm{H} & 3.91943300 & 2.72986900 & -0.33912700\end{array}$

Int11,"

33

$\begin{array}{lccc}\mathrm{Fe} & 10.30894300 & 7.01219600 & 2.30363500 \\ \mathrm{Cl} & 12.10672300 & 8.37199500 & 2.07982300 \\ \mathrm{~N} & 11.28093600 & 6.27380100 & 3.91143300 \\ \mathrm{C} & 12.39431000 & 5.56442300 & 4.00948600 \\ \mathrm{H} & 12.93851100 & 5.37965100 & 3.09067400 \\ \mathrm{~N} & 9.43688100 & 8.12096200 & 3.66295700 \\ \mathrm{C} & 12.88584100 & 5.10949900 & 5.24358800 \\ \mathrm{H} & 13.80513700 & 4.53655000 & 5.26845200 \\ \mathrm{~N} & 9.56297700 & 8.04136100 & 0.78299200 \\ \mathrm{C} & 12.20597200 & 5.41947100 & 6.40107900 \\ \mathrm{H} & 12.56958400 & 5.07887100 & 7.36573500 \\ \mathrm{~N} & 10.91602400 & 5.79817100 & 0.84122800 \\ \mathrm{C} & 11.05792500 & 6.23615700 & 6.32774400 \\ \mathrm{C} & 10.65779200 & 6.65466600 & 5.05078200 \\ \mathrm{C} & 10.31175900 & 6.69058500 & 7.46893100 \\ \mathrm{H} & 10.61364600 & 6.35252800 & 8.45500000 \\ \mathrm{C} & 9.27061700 & 7.55218000 & 7.32602600 \\ \mathrm{H} & 8.72310300 & 7.90296800 & 8.19449000 \\ \mathrm{C} & 8.91556500 & 8.06499700 & 6.03333300 \\ \mathrm{C} & 9.61514000 & 7.61922000 & 4.90799800 \\ \mathrm{C} & 7.91252400 & 9.02626600 & 5.79420400 \\ \mathrm{H} & 7.29023700 & 9.37885500 & 6.61085500 \\ \mathrm{C} & 7.74927900 & 9.53394000 & 4.53032200 \\ \mathrm{H} & 6.97804200 & 10.27161100 & 4.35449500 \\ \mathrm{C} & 8.58284800 & 9.11347600 & 3.45924500 \\ \mathrm{C} & 8.53957200 & 9.81456800 & 2.15215900 \\ \mathrm{C} & 8.08832800 & 11.12484300 & 2.17009000 \\ \mathrm{H} & 7.84311800 & 11.58829000 & 3.11776000 \\ \mathrm{C} & 7.97223000 & 11.92394900 & 1.01978900 \\ \mathrm{H} & 7.62170000 & 12.94564600 & 1.10873600 \\ & & & \end{array}$




\begin{tabular}{|c|c|c|c|}
\hline $\mathrm{C}$ & 8.32676000 & 11.40319400 & -0.19148400 \\
\hline $\mathrm{H}$ & 8.25523300 & 11.99371700 & -1.09926700 \\
\hline $\mathrm{C}$ & 8.84571800 & 10.08861200 & -0.26824700 \\
\hline $\mathrm{C}$ & 8.98151100 & 9.27708200 & 0.89695700 \\
\hline $\mathrm{C}$ & 9.28185900 & 9.58089300 & -1.51050800 \\
\hline $\mathrm{H}$ & 9.19713000 & 10.20299700 & -2.39620400 \\
\hline $\mathrm{C}$ & 9.84140800 & 8.33776800 & -1.58494800 \\
\hline $\mathrm{H}$ & 10.21996200 & 7.96081200 & -2.52553300 \\
\hline $\mathrm{C}$ & 9.98624000 & 7.59782300 & -0.39944400 \\
\hline $\mathrm{C}$ & 10.65426400 & 6.27993100 & -0.38516000 \\
\hline $\mathrm{C}$ & 10.96327000 & 5.55210400 & -1.53136500 \\
\hline $\mathrm{H}$ & 10.76258100 & 5.95205500 & -2.51708800 \\
\hline $\mathrm{C}$ & 11.54454000 & 4.29685300 & -1.39922600 \\
\hline $\mathrm{H}$ & 11.81653000 & 3.72500000 & -2.27974900 \\
\hline $\mathrm{C}$ & 11.76212300 & 3.78653900 & -0.12509500 \\
\hline $\mathrm{H}$ & 12.19617100 & 2.80534300 & 0.02489100 \\
\hline $\mathrm{C}$ & 11.42136500 & 4.57061800 & 0.96783100 \\
\hline $\mathrm{H}$ & 11.54991700 & 4.20884700 & 1.97979000 \\
\hline $\mathrm{O}$ & 8.97672000 & 5.86646000 & 2.51665700 \\
\hline $\mathrm{O}$ & 6.08192400 & 3.61338600 & 3.05555200 \\
\hline $\mathrm{Fe}$ & 7.54613100 & 4.86193400 & 2.71425700 \\
\hline $\mathrm{N}$ & 8.30714200 & 4.20400000 & 4.47530800 \\
\hline $\mathrm{N}$ & 6.54301000 & 6.07956400 & 3.87862700 \\
\hline $\mathrm{N}$ & 6.86208500 & 5.68533200 & 1.04448400 \\
\hline $\mathrm{N}$ & 8.16871000 & 3.43762900 & 1.44417700 \\
\hline $\mathrm{C}$ & 9.34605900 & 3.43566700 & 4.76217700 \\
\hline $\mathrm{H}$ & 9.96607100 & 3.12578700 & 3.92886300 \\
\hline $\mathrm{C}$ & 9.65224700 & 3.03324900 & 6.07109500 \\
\hline $\mathrm{H}$ & 10.50427200 & 2.38677800 & 6.24399100 \\
\hline $\mathrm{C}$ & 8.85150200 & 3.45282100 & 7.11082400 \\
\hline $\mathrm{H}$ & 9.04681100 & 3.12932200 & 8.12857400 \\
\hline $\mathrm{C}$ & 7.78232000 & 4.33258100 & 6.84382300 \\
\hline $\mathrm{C}$ & 7.57601500 & 4.69621700 & 5.50426300 \\
\hline $\mathrm{C}$ & 6.92022600 & 4.88095800 & 7.85464900 \\
\hline $\mathrm{H}$ & 7.06416000 & 4.57235100 & 8.88492400 \\
\hline $\mathrm{C}$ & 5.94025500 & 5.76421400 & 7.53169600 \\
\hline $\mathrm{H}$ & 5.28508300 & 6.16459800 & 8.29851400 \\
\hline $\mathrm{C}$ & 5.76036900 & 6.19808500 & 6.17438800 \\
\hline $\mathrm{C}$ & 6.58713300 & 5.67603500 & 5.17376900 \\
\hline $\mathrm{C}$ & 4.78088100 & 7.12059700 & 5.75133800 \\
\hline $\mathrm{H}$ & 4.05743000 & 7.50999900 & 6.46133600 \\
\hline $\mathrm{C}$ & 4.74260400 & 7.51739500 & 4.43750700 \\
\hline $\mathrm{H}$ & 3.96729800 & 8.19945500 & 4.11479300 \\
\hline $\mathrm{C}$ & 5.68880300 & 7.02522200 & 3.50021600 \\
\hline $\mathrm{C}$ & 5.74233000 & 7.58466000 & 2.12604200 \\
\hline $\mathrm{C}$ & 5.25659500 & 8.87094900 & 1.95822600 \\
\hline
\end{tabular}




$\begin{array}{lccc}\mathrm{H} & 4.91166900 & 9.42397700 & 2.82349300 \\ \mathrm{C} & 5.20128500 & 9.52675200 & 0.71572400 \\ \mathrm{H} & 4.80356500 & 10.53293500 & 0.65710500 \\ \mathrm{C} & 5.64306600 & 8.87770500 & -0.40120000 \\ \mathrm{H} & 5.59402700 & 9.34642500 & -1.37872500 \\ \mathrm{C} & 6.18842100 & 7.57580500 & -0.29120800 \\ \mathrm{C} & 6.27463600 & 6.91654300 & 0.97060000 \\ \mathrm{C} & 6.67670900 & 6.91533400 & -1.43855200 \\ \mathrm{H} & 6.60447100 & 7.40688000 & -2.40357500 \\ \mathrm{C} & 7.24080300 & 5.67606600 & -1.32772500 \\ \mathrm{H} & 7.63325200 & 5.17037600 & -2.19977900 \\ \mathrm{C} & 7.33316900 & 5.09366200 & -0.05154400 \\ \mathrm{C} & 7.96908900 & 3.77310700 & 0.15589900 \\ \mathrm{C} & 8.28256800 & 2.89754100 & -0.87904200 \\ \mathrm{H} & 8.10564200 & 3.16833300 & -1.91202600 \\ \mathrm{C} & 8.79745900 & 1.64215300 & -0.57387500 \\ \mathrm{H} & 9.04392300 & 0.94410700 & -1.36666300 \\ \mathrm{C} & 8.95556900 & 1.28746300 & 0.75940300 \\ \mathrm{H} & 9.31893900 & 0.30752900 & 1.04583700 \\ \mathrm{C} & 8.61676700 & 2.21461900 & 1.73647000 \\ \mathrm{H} & 8.67860500 & 1.96140800 & 2.78658900 \\ \mathrm{O} & 4.80901600 & 4.05729300 & 2.48009600 \\ \mathrm{H} & 4.54711600 & 3.27383700 & 1.82353500 \\ \mathrm{H} & 4.20479000 & 4.05049100 & 3.23951800 \\ \mathrm{O} & 4.30009200 & 2.20768100 & 0.92422400 \\ \mathrm{H} & 4.44526900 & 1.30859500 & 1.23901700 \\ \mathrm{H} & 3.51432300 & 2.19476500 & 0.36596700\end{array}$

Complex 1'

46

$\begin{array}{lccc}\mathrm{Fe} & 5.85454000 & 10.53927900 & 3.82391500 \\ \mathrm{Cl} & 6.74007300 & 12.60907400 & 3.88507800 \\ \mathrm{~N} & 6.95831100 & 10.24247500 & 5.47756500 \\ \mathrm{C} & 8.27709300 & 10.15749400 & 5.64446400 \\ \mathrm{H} & 8.89495100 & 10.28070600 & 4.76359500 \\ \mathrm{~N} & 4.47694600 & 11.02498100 & 5.14266600 \\ \mathrm{C} & 8.86183700 & 9.97106700 & 6.90319300 \\ \mathrm{H} & 9.94135900 & 9.91026900 & 6.98132900 \\ \mathrm{~N} & 4.83197100 & 11.25153400 & 2.29483900 \\ \mathrm{C} & 8.05872300 & 9.89391700 & 8.02520700 \\ \mathrm{H} & 8.49402800 & 9.75259000 & 9.01009000 \\ \mathrm{~N} & 7.09597400 & 9.94561500 & 2.40586800 \\ \mathrm{C} & 6.66789700 & 10.06685200 & 7.87657900 \\ \mathrm{C} & 6.17850400 & 10.26081500 & 6.57809500 \\ \mathrm{C} & 5.74095400 & 10.11135800 & 8.97604100 \\ \mathrm{H} & 6.11828300 & 9.94241200 & 9.98039800\end{array}$




\begin{tabular}{|c|c|c|c|}
\hline $\mathrm{C}$ & 4.41208100 & 10.39125600 & 8.78281300 \\
\hline $\mathrm{H}$ & 3.73439000 & 10.43708500 & 9.62863200 \\
\hline $\mathrm{C}$ & 3.92289900 & 10.69481500 & 7.48710400 \\
\hline $\mathrm{C}$ & 4.81021200 & 10.64268800 & 6.37893600 \\
\hline $\mathrm{C}$ & 2.59253900 & 11.07693700 & 7.20025200 \\
\hline $\mathrm{H}$ & 1.84492700 & 11.07776200 & 7.98697300 \\
\hline $\mathrm{C}$ & 2.26869100 & 11.48110700 & 5.93504300 \\
\hline $\mathrm{H}$ & 1.24716200 & 11.76789500 & 5.73084700 \\
\hline $\mathrm{C}$ & 3.24883100 & 11.52786100 & 4.89477100 \\
\hline $\mathrm{C}$ & 2.94918100 & 12.15265000 & 3.62219000 \\
\hline $\mathrm{C}$ & 1.85477500 & 13.05653200 & 3.61483800 \\
\hline $\mathrm{H}$ & 1.33468600 & 13.26313100 & 4.54042700 \\
\hline $\mathrm{C}$ & 1.44488900 & 13.76514100 & 2.49816300 \\
\hline $\mathrm{H}$ & 0.61737400 & 14.46237700 & 2.56543900 \\
\hline $\mathrm{C}$ & 2.13146900 & 13.58975800 & 1.31153100 \\
\hline $\mathrm{H}$ & 1.83825200 & 14.13656500 & 0.41945500 \\
\hline $\mathrm{C}$ & 3.26138000 & 12.73238700 & 1.24872300 \\
\hline $\mathrm{C}$ & 3.70923900 & 12.01259800 & 2.39108900 \\
\hline $\mathrm{C}$ & 3.98367400 & 12.64020700 & 0.04278900 \\
\hline $\mathrm{H}$ & 3.65892500 & 13.20833100 & -0.82390900 \\
\hline $\mathrm{C}$ & 5.11185900 & 11.86573800 & -0.01440000 \\
\hline $\mathrm{H}$ & 5.70026100 & 11.82367000 & -0.92137200 \\
\hline $\mathrm{C}$ & 5.52034400 & 11.19061200 & 1.14272900 \\
\hline $\mathrm{C}$ & 6.75135400 & 10.38194800 & 1.17908000 \\
\hline $\mathrm{C}$ & 7.49240300 & 10.03689700 & 0.05418400 \\
\hline $\mathrm{H}$ & 7.22631300 & 10.40381700 & -0.92912600 \\
\hline $\mathrm{C}$ & 8.60059100 & 9.20687300 & 0.20069900 \\
\hline $\mathrm{H}$ & 9.20863100 & 8.94878600 & -0.65994100 \\
\hline $\mathrm{C}$ & 8.90133000 & 8.70554600 & 1.46080000 \\
\hline $\mathrm{H}$ & 9.73766100 & 8.03410800 & 1.61677100 \\
\hline $\mathrm{C}$ & 8.11326100 & 9.09205200 & 2.53759300 \\
\hline $\mathrm{H}$ & 8.29287900 & 8.69905100 & 3.52920500 \\
\hline $\mathrm{O}$ & 5.22251700 & 9.01451400 & 3.81293400 \\
\hline $\mathrm{O}$ & 3.27469200 & 5.93137800 & 3.81971100 \\
\hline $\mathrm{Fe}$ & 4.11688100 & 7.30546900 & 3.80566400 \\
\hline $\mathrm{N}$ & 4.89326800 & 7.05967100 & 5.66188500 \\
\hline $\mathrm{N}$ & 2.63481000 & 8.13565000 & 4.82081100 \\
\hline $\mathrm{N}$ & 3.48754200 & 7.96696200 & 2.06574700 \\
\hline $\mathrm{N}$ & 5.47826700 & 6.38175900 & 2.69563600 \\
\hline $\mathrm{C}$ & 6.10244100 & 6.70327400 & 6.07437200 \\
\hline $\mathrm{H}$ & 6.87417900 & 6.62196500 & 5.31837700 \\
\hline $\mathrm{C}$ & 6.38755200 & 6.42464700 & 7.41939600 \\
\hline $\mathrm{H}$ & 7.38491600 & 6.10327200 & 7.69638000 \\
\hline $\mathrm{C}$ & 5.38102400 & 6.51517000 & 8.35793700 \\
\hline $\mathrm{H}$ & 5.56443100 & 6.25055300 & 9.39521500 \\
\hline $\mathrm{C}$ & 4.10240700 & 6.95779700 & 7.95593800 \\
\hline
\end{tabular}




$\begin{array}{lccc}\mathrm{C} & 3.92652600 & 7.24530000 & 6.59408800 \\ \mathrm{C} & 2.98509900 & 7.11475300 & 8.84759800 \\ \mathrm{H} & 3.11104500 & 6.85269800 & 9.89342000 \\ \mathrm{C} & 1.77678600 & 7.54090700 & 8.38720900 \\ \mathrm{H} & 0.92775600 & 7.61316000 & 9.05952900 \\ \mathrm{C} & 1.59823200 & 7.88731900 & 7.00715800 \\ \mathrm{C} & 2.68340800 & 7.77236900 & 6.12535700 \\ \mathrm{C} & 0.37735200 & 8.30422900 & 6.43415900 \\ \mathrm{H} & -0.52882100 & 8.32130300 & 7.03252700 \\ \mathrm{C} & 0.33598200 & 8.65970000 & 5.10893700 \\ \mathrm{H} & -0.61842300 & 8.91877000 & 4.67076100 \\ \mathrm{C} & 1.50662200 & 8.62324200 & 4.30181300 \\ \mathrm{C} & 1.47963000 & 9.13174300 & 2.91172800 \\ \mathrm{C} & 0.45914100 & 10.02406200 & 2.59441600 \\ \mathrm{H} & -0.22797400 & 10.34002800 & 3.36949800 \\ \mathrm{C} & 0.24406600 & 10.54252200 & 1.30887100 \\ \mathrm{H} & -0.60069900 & 11.19700000 & 1.12637700 \\ \mathrm{C} & 1.08842100 & 10.17774600 & 0.29624300 \\ \mathrm{H} & 0.92838300 & 10.52687500 & -0.71941800 \\ \mathrm{C} & 2.18278900 & 9.31734400 & 0.56151300 \\ \mathrm{C} & 2.40985000 & 8.78583100 & 1.86597900 \\ \mathrm{C} & 3.06645600 & 8.97314400 & -0.48390200 \\ \mathrm{H} & 2.87963300 & 9.35246900 & -1.48412800 \\ \mathrm{C} & 4.13421100 & 8.15538200 & -0.23960400 \\ \mathrm{H} & 4.80747400 & 7.87847800 & -1.03962700 \\ \mathrm{C} & 4.31710200 & 7.66099600 & 1.06194500 \\ \mathrm{C} & 5.40755300 & 6.71999300 & 1.39185800 \\ \mathrm{C} & 6.22488500 & 6.11603200 & 0.44394100 \\ \mathrm{H} & 6.15702300 & 6.37518800 & -0.60491800 \\ \mathrm{C} & 7.09996000 & 5.10809900 & 0.84421200 \\ \mathrm{H} & 7.72872000 & 4.60805600 & 0.11507000 \\ \mathrm{C} & 7.10594000 & 4.71324400 & 2.17507900 \\ \mathrm{H} & 7.72137900 & 3.88977200 & 2.51848900 \\ \mathrm{C} & 6.26959000 & 5.37162900 & 3.06899500 \\ \mathrm{H} & 6.19623300 & 5.04984900 & 4.09959100\end{array}$

\section{TS1 1 ,}

34

$\begin{array}{llll}\mathrm{Fe} & 5.81321600 & 10.59854400 & 3.81392100 \\ \mathrm{Cl} & 6.78930500 & 12.62247500 & 3.83066700 \\ \mathrm{~N} & 6.89010500 & 10.27047300 & 5.48172000 \\ \mathrm{C} & 8.19496400 & 10.13386000 & 5.66722200 \\ \mathrm{H} & 8.83331600 & 10.26622000 & 4.80220500 \\ \mathrm{~N} & 4.44247800 & 11.12997700 & 5.10172600 \\ \mathrm{C} & 8.74631200 & 9.88026800 & 6.93291400 \\ \mathrm{H} & 9.82076500 & 9.77895300 & 7.02952600\end{array}$




\begin{tabular}{|c|c|c|c|}
\hline $\mathrm{N}$ & 4.81917000 & 11.29627100 & 2.25425000 \\
\hline $\mathrm{C}$ & 7.91841000 & 9.78903700 & 8.03051800 \\
\hline $\mathrm{H}$ & 8.32668400 & 9.59358100 & 9.01736100 \\
\hline $\mathrm{N}$ & 7.05355200 & 9.93335500 & 2.40671300 \\
\hline $\mathrm{C}$ & 6.53444300 & 10.01311100 & 7.86958800 \\
\hline $\mathrm{C}$ & 6.08170200 & 10.27673100 & 6.56893200 \\
\hline $\mathrm{C}$ & 5.58480100 & 10.03179100 & 8.94742600 \\
\hline $\mathrm{H}$ & 5.93364700 & 9.81276200 & 9.95133300 \\
\hline $\mathrm{C}$ & 4.28166500 & 10.34867200 & 8.72514200 \\
\hline $\mathrm{H}$ & 3.57299800 & 10.37406500 & 9.54607400 \\
\hline $\mathrm{C}$ & 3.83060200 & 10.71654900 & 7.41383000 \\
\hline $\mathrm{C}$ & 4.73674000 & 10.68979200 & 6.35064200 \\
\hline $\mathrm{C}$ & 2.52052900 & 11.13181200 & 7.09672300 \\
\hline $\mathrm{H}$ & 1.74614900 & 11.11919800 & 7.85732200 \\
\hline $\mathrm{C}$ & 2.23833300 & 11.57456600 & 5.82977200 \\
\hline $\mathrm{H}$ & 1.23033800 & 11.88627500 & 5.59294700 \\
\hline $\mathrm{C}$ & 3.24745500 & 11.63974700 & 4.83189300 \\
\hline $\mathrm{C}$ & 2.98424600 & 12.31716400 & 3.54230200 \\
\hline $\mathrm{C}$ & 1.97886800 & 13.27214700 & 3.53797900 \\
\hline $\mathrm{H}$ & 1.47187200 & 13.51459700 & 4.46363300 \\
\hline $\mathrm{C}$ & 1.60023200 & 13.99712800 & 2.39555700 \\
\hline $\mathrm{H}$ & 0.81150700 & 14.73710200 & 2.46552000 \\
\hline $\mathrm{C}$ & 2.25189800 & 13.76925400 & 1.21765800 \\
\hline $\mathrm{H}$ & 1.98869200 & 14.31346600 & 0.31634700 \\
\hline $\mathrm{C}$ & 3.32372800 & 12.84534500 & 1.17100800 \\
\hline $\mathrm{C}$ & 3.72373200 & 12.11682200 & 2.33108700 \\
\hline $\mathrm{C}$ & 4.04388800 & 12.67412500 & -0.02891900 \\
\hline $\mathrm{H}$ & 3.75155500 & 13.23889800 & -0.90877700 \\
\hline $\mathrm{C}$ & 5.12375700 & 11.83821600 & -0.06683900 \\
\hline $\mathrm{H}$ & 5.70931500 & 11.73714700 & -0.9706260 \\
\hline $\mathrm{C}$ & 5.49959900 & 11.17717200 & 1.11214500 \\
\hline $\mathrm{C}$ & 6.70175000 & 10.32427400 & 1.16975200 \\
\hline $\mathrm{C}$ & 7.41172900 & 9.89841000 & 0.05089600 \\
\hline $\mathrm{H}$ & 7.13501900 & 10.22722000 & -0.9425580 \\
\hline $\mathrm{C}$ & 8.49069700 & 9.03884100 & 0.21928100 \\
\hline $\mathrm{H}$ & 9.07045400 & 8.71486500 & -0.63820800 \\
\hline $\mathrm{C}$ & 8.80159000 & 8.59061000 & 1.49782800 \\
\hline $\mathrm{H}$ & 9.61632200 & 7.89828900 & 1.67260400 \\
\hline $\mathrm{C}$ & 8.04678400 & 9.05543100 & 2.56413600 \\
\hline $\mathrm{H}$ & 8.23010900 & 8.70931200 & 3.57219900 \\
\hline $\mathrm{O}$ & 5.16587100 & 9.07545700 & 3.82631500 \\
\hline $\mathrm{O}$ & 3.45614400 & 5.89137500 & 3.87762900 \\
\hline $\mathrm{Fe}$ & 4.16544700 & 7.35963100 & 3.82448700 \\
\hline $\mathrm{N}$ & 4.95695500 & 7.10906100 & 5.67153300 \\
\hline $\mathrm{N}$ & 2.65595300 & 8.09544500 & 4.84917100 \\
\hline $\mathrm{N}$ & 3.48394300 & 7.99648700 & $2.0978250 \mathrm{C}$ \\
\hline
\end{tabular}




\begin{tabular}{|c|c|c|c|}
\hline $\mathrm{N}$ & 5.57043800 & 6.50741900 & 2.68987200 \\
\hline $\mathrm{C}$ & 6.18593800 & 6.82851800 & 6.07887400 \\
\hline $\mathrm{H}$ & 6.96440200 & 6.83209000 & 5.32560400 \\
\hline $\mathrm{C}$ & 6.48394600 & 6.53599400 & 7.41746400 \\
\hline $\mathrm{H}$ & 7.50206500 & 6.28825300 & 7.69287600 \\
\hline $\mathrm{C}$ & 5.47102900 & 6.53806200 & 8.35277500 \\
\hline $\mathrm{H}$ & 5.67031800 & 6.27264500 & 9.38648100 \\
\hline $\mathrm{C}$ & 4.16785900 & 6.90619500 & 7.95846800 \\
\hline $\mathrm{C}$ & 3.97760300 & 7.21202900 & 6.60283400 \\
\hline $\mathrm{C}$ & 3.04540200 & 6.99238600 & 8.85096200 \\
\hline $\mathrm{H}$ & 3.18527700 & 6.72159400 & 9.89226300 \\
\hline $\mathrm{C}$ & 1.82541100 & 7.38598200 & 8.39931500 \\
\hline $\mathrm{H}$ & 0.97579800 & 7.42353900 & 9.07328300 \\
\hline $\mathrm{C}$ & 1.63378000 & 7.76147400 & 7.02654600 \\
\hline $\mathrm{C}$ & 2.71540100 & 7.69468600 & 6.14508900 \\
\hline $\mathrm{C}$ & 0.40578500 & 8.17667800 & 6.46887400 \\
\hline $\mathrm{H}$ & -0.49696500 & 8.16836600 & 7.07199500 \\
\hline $\mathrm{C}$ & 0.35482800 & 8.56979900 & 5.15545700 \\
\hline $\mathrm{H}$ & -0.59968700 & 8.83728700 & 4.72297800 \\
\hline $\mathrm{C}$ & 1.52332000 & 8.57823900 & 4.35018100 \\
\hline $\mathrm{C}$ & 1.48892000 & 9.14324100 & 2.98112600 \\
\hline $\mathrm{C}$ & 0.49319800 & 10.06911200 & 2.71329300 \\
\hline $\mathrm{H}$ & -0.17237300 & 10.37621400 & 3.51053400 \\
\hline $\mathrm{C}$ & 0.28993900 & 10.65278600 & 1.45161000 \\
\hline $\mathrm{H}$ & -0.52173000 & 11.35626900 & 1.30905400 \\
\hline $\mathrm{C}$ & 1.11006100 & 10.30158500 & 0.41824200 \\
\hline $\mathrm{H}$ & 0.96263000 & 10.70573500 & -0.57807600 \\
\hline $\mathrm{C}$ & 2.17833500 & 9.39949700 & 0.63954200 \\
\hline $\mathrm{C}$ & 2.40600100 & 8.82462900 & 1.92498300 \\
\hline $\mathrm{C}$ & 3.04381300 & 9.06596200 & -0.42284500 \\
\hline $\mathrm{H}$ & 2.85733000 & 9.48193100 & -1.40794100 \\
\hline $\mathrm{C}$ & 4.10148800 & 8.22831400 & -0.21008900 \\
\hline $\mathrm{H}$ & 4.76985300 & 7.96863700 & -1.01942200 \\
\hline $\mathrm{C}$ & 4.30068000 & 7.71455400 & 1.08037000 \\
\hline $\mathrm{C}$ & 5.41798900 & 6.79838200 & 1.38448500 \\
\hline $\mathrm{C}$ & 6.20077800 & 6.18852400 & 0.41165600 \\
\hline $\mathrm{H}$ & 6.05739200 & 6.40658900 & -0.63851700 \\
\hline $\mathrm{C}$ & 7.13793500 & 5.23439200 & 0.79444200 \\
\hline $\mathrm{H}$ & 7.74319000 & 4.73201200 & 0.04770200 \\
\hline $\mathrm{C}$ & 7.24354100 & 4.89715500 & 2.13699300 \\
\hline $\mathrm{H}$ & 7.91815500 & 4.11950100 & 2.47482500 \\
\hline $\mathrm{C}$ & 6.42821700 & 5.54957500 & 3.05164000 \\
\hline $\mathrm{H}$ & 6.42877500 & 5.26403600 & 4.09489800 \\
\hline $\mathrm{O}$ & 1.66726600 & 5.80708600 & 3.05866500 \\
\hline $\mathrm{N}$ & 1.76522700 & 5.39563200 & 1.78339400 \\
\hline $\mathrm{O}$ & 2.83560800 & 5.08562800 & 1.32945200 \\
\hline
\end{tabular}


$\begin{array}{llll}\text { O } & 0.67944300 & 5.42474500 & 1.25484500\end{array}$

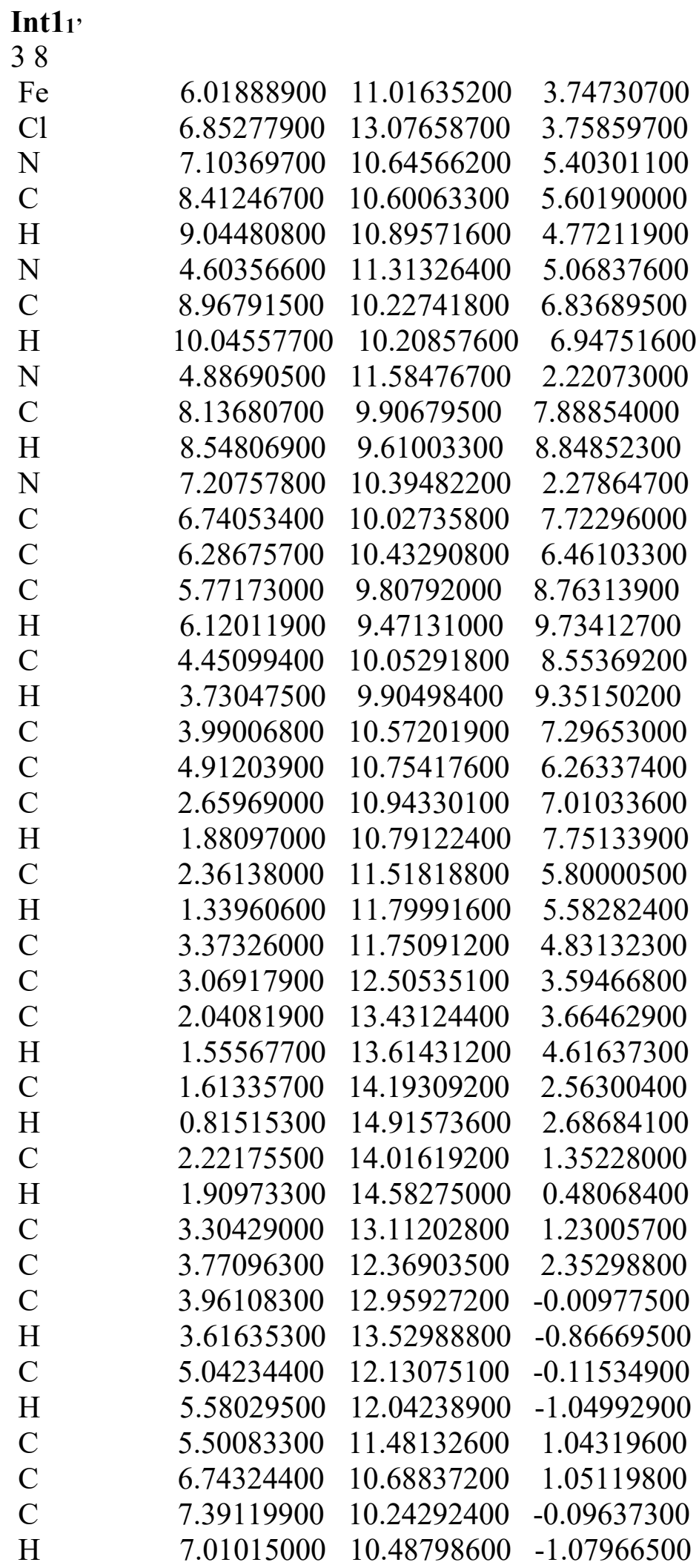




\begin{tabular}{|c|c|c|c|}
\hline $\mathrm{C}$ & 8.54273300 & 9.47531500 & 0.03198700 \\
\hline $\mathrm{H}$ & 9.07579900 & 9.13587800 & -0.84938900 \\
\hline $\mathrm{C}$ & 8.99333300 & 9.14727000 & 1.30497500 \\
\hline $\mathrm{H}$ & 9.88012800 & 8.54268000 & 1.45256700 \\
\hline $\mathrm{C}$ & 8.28671900 & 9.61892800 & 2.40188600 \\
\hline $\mathrm{H}$ & 8.58333300 & 9.35984800 & 3.40887700 \\
\hline $\mathrm{O}$ & 5.46798900 & 9.23737200 & 3.77148100 \\
\hline $\mathrm{O}$ & 3.31923200 & 5.35079400 & 4.03451600 \\
\hline $\mathrm{Fe}$ & 4.53986600 & 7.78749500 & 3.87163800 \\
\hline $\mathrm{N}$ & 4.94369400 & 7.14508100 & 5.86802300 \\
\hline $\mathrm{N}$ & 2.66468600 & 8.13202000 & 4.79108300 \\
\hline $\mathrm{N}$ & 3.50452800 & 7.98485800 & 2.00623600 \\
\hline $\mathrm{N}$ & 5.57933200 & 6.40705700 & 2.62407700 \\
\hline $\mathrm{C}$ & 6.09815500 & 6.77693800 & 6.40763100 \\
\hline $\mathrm{H}$ & 6.97900800 & 6.86891500 & 5.77904900 \\
\hline $\mathrm{C}$ & 6.19798900 & 6.29513900 & 7.71925900 \\
\hline $\mathrm{H}$ & 7.16180400 & 5.98893500 & 8.10809000 \\
\hline $\mathrm{C}$ & 5.05550800 & 6.20651000 & 8.48451600 \\
\hline $\mathrm{H}$ & 5.09686400 & 5.81836900 & 9.49773500 \\
\hline $\mathrm{C}$ & 3.82204200 & 6.63494200 & 7.95029600 \\
\hline $\mathrm{C}$ & 3.82155200 & 7.11158400 & 6.62756300 \\
\hline $\mathrm{C}$ & 2.59361700 & 6.61011400 & 8.69282400 \\
\hline $\mathrm{H}$ & 2.60453000 & 6.22570300 & 9.70745600 \\
\hline $\mathrm{C}$ & 1.43735300 & 7.04714600 & 8.13260400 \\
\hline $\mathrm{H}$ & 0.50713900 & 7.01389800 & 8.69041700 \\
\hline $\mathrm{C}$ & 1.41840900 & 7.56040500 & 6.79229100 \\
\hline $\mathrm{C}$ & 2.60318300 & 7.60961300 & 6.04435900 \\
\hline $\mathrm{C}$ & 0.24702300 & 8.01313000 & 6.14901200 \\
\hline $\mathrm{H}$ & -0.70994300 & 7.94451300 & 6.65760400 \\
\hline $\mathrm{C}$ & 0.31935600 & 8.51835300 & 4.87579300 \\
\hline $\mathrm{H}$ & -0.58750800 & 8.81783300 & 4.36675700 \\
\hline $\mathrm{C}$ & 1.56751300 & 8.61468100 & 4.21071300 \\
\hline $\mathrm{C}$ & 1.62719900 & 9.27418200 & 2.87904500 \\
\hline $\mathrm{C}$ & 0.70262800 & 10.27364000 & 2.62469100 \\
\hline $\mathrm{H}$ & 0.04545400 & 10.60392600 & 3.42143500 \\
\hline $\mathrm{C}$ & 0.56285400 & 10.89184200 & 1.36863100 \\
\hline $\mathrm{H}$ & -0.18863300 & 11.66042000 & 1.23227900 \\
\hline $\mathrm{C}$ & 1.35989600 & 10.49757400 & 0.32957700 \\
\hline $\mathrm{H}$ & 1.24627900 & 10.93157800 & -0.65873100 \\
\hline $\mathrm{C}$ & 2.34834100 & 9.50599700 & 0.53679800 \\
\hline $\mathrm{C}$ & 2.51824700 & 8.90769500 & 1.81741700 \\
\hline $\mathrm{C}$ & 3.18806000 & 9.08061200 & -0.51754300 \\
\hline $\mathrm{H}$ & 3.06838800 & 9.52565600 & -1.50065700 \\
\hline $\mathrm{C}$ & 4.13006400 & 8.11039900 & -0.30443300 \\
\hline $\mathrm{H}$ & 4.77399400 & 7.77838100 & -1.10862700 \\
\hline $\mathrm{C}$ & 4.26383600 & 7.58191100 & 0.99366000 \\
\hline
\end{tabular}




$\begin{array}{lccc}\mathrm{C} & 5.27750500 & 6.54303500 & 1.31767000 \\ \mathrm{C} & 5.84520900 & 5.71554000 & 0.35539600 \\ \mathrm{H} & 5.57003800 & 5.80615100 & -0.68827900 \\ \mathrm{C} & 6.74375000 & 4.72925500 & 0.75405300 \\ \mathrm{H} & 7.18767800 & 4.06650500 & 0.01908100 \\ \mathrm{C} & 7.04396400 & 4.59199600 & 2.10366100 \\ \mathrm{H} & 7.72496100 & 3.82693100 & 2.45690800 \\ \mathrm{C} & 6.43267900 & 5.45154400 & 3.00635200 \\ \mathrm{H} & 6.61533900 & 5.36315200 & 4.07129200 \\ \mathrm{O} & 2.43368900 & 3.00285900 & 4.12473400 \\ \mathrm{~N} & 1.67762500 & 3.63701800 & 3.49389900 \\ \mathrm{O} & 2.24757500 & 5.29230400 & 3.39035500 \\ \mathrm{O} & 0.66654200 & 3.53211700 & 2.90950900\end{array}$

Decomposition mechanism

TS1 1

35

$\begin{array}{lccc}\mathrm{Fe} & 10.28323700 & 7.03841100 & 2.29158100 \\ \mathrm{Cl} & 11.87220500 & 8.67748400 & 2.15412100 \\ \mathrm{~N} & 11.64331400 & 6.14390600 & 3.46616000 \\ \mathrm{C} & 12.81486200 & 5.59623400 & 3.17664000 \\ \mathrm{H} & 13.15242000 & 5.67012000 & 2.15041500 \\ \mathrm{~N} & 9.60835100 & 7.72141600 & 4.00031400 \\ \mathrm{C} & 13.60255600 & 4.96898100 & 4.15386300 \\ \mathrm{H} & 14.55212000 & 4.53284900 & 3.86761100 \\ \mathrm{~N} & 9.21573700 & 8.28085700 & 1.19699300 \\ \mathrm{C} & 13.16253600 & 4.92888600 & 5.46008000 \\ \mathrm{H} & 13.75528300 & 4.44216900 & 6.22853600 \\ \mathrm{~N} & 10.79472300 & 6.30907900 & 0.51258100 \\ \mathrm{C} & 11.95841500 & 5.58063500 & 5.80334900 \\ \mathrm{C} & 11.25272500 & 6.20023100 & 4.76114600 \\ \mathrm{C} & 11.44725600 & 5.68821800 & 7.14234400 \\ \mathrm{H} & 11.98544700 & 5.19658300 & 7.94610300 \\ \mathrm{C} & 10.33733600 & 6.42666500 & 7.40474800 \\ \mathrm{H} & 9.97107600 & 6.52854600 & 8.42114800 \\ \mathrm{C} & 9.66237300 & 7.13954400 & 6.35763100 \\ \mathrm{C} & 10.12259900 & 7.02325400 & 5.04394900 \\ \mathrm{C} & 8.55171200 & 7.98484700 & 6.54681600 \\ \mathrm{H} & 8.11273700 & 8.09499600 & 7.53295600 \\ \mathrm{C} & 8.03213100 & 8.67073700 & 5.48018200 \\ \mathrm{H} & 7.16399800 & 9.29213500 & 5.64255900 \\ \mathrm{C} & 8.61037200 & 8.57787800 & 4.18487100 \\ \mathrm{C} & 8.15014600 & 9.46517800 & 3.08546500 \\ \mathrm{C} & 7.42228900 & 10.58690300 & 3.45795600 \\ \mathrm{H} & 7.23404500 & 10.78031200 & 4.50497900 \\ \mathrm{C} & 6.94676900 & 11.55246100 & 2.55592400\end{array}$




\begin{tabular}{|c|c|c|c|}
\hline $\mathrm{H}$ & 6.38361500 & 12.40142500 & 2.92532700 \\
\hline $\mathrm{C}$ & 7.24611500 & 11.42575700 & 1.23056300 \\
\hline $\mathrm{H}$ & 6.92537200 & 12.16979300 & 0.50854800 \\
\hline $\mathrm{C}$ & 8.02479000 & 10.33036300 & 0.78682200 \\
\hline $\mathrm{C}$ & 8.47423400 & 9.32034100 & 1.69150600 \\
\hline $\mathrm{C}$ & 8.39846400 & 10.25814400 & -0.57095300 \\
\hline $\mathrm{H}$ & 8.09194700 & 11.04989300 & -1.24736100 \\
\hline $\mathrm{C}$ & 9.15727500 & 9.21571700 & -1.01745100 \\
\hline $\mathrm{H}$ & 9.47958900 & 9.17096300 & -2.04873600 \\
\hline $\mathrm{C}$ & 9.54692100 & 8.23303300 & -0.09610700 \\
\hline $\mathrm{C}$ & 10.36869200 & 7.07841700 & -0.50410800 \\
\hline $\mathrm{C}$ & 10.65036300 & 6.74789700 & -1.82594500 \\
\hline $\mathrm{H}$ & 10.32292900 & 7.37970800 & -2.64148000 \\
\hline $\mathrm{C}$ & 11.36680300 & 5.58731400 & -2.09567300 \\
\hline $\mathrm{H}$ & 11.61967800 & 5.32647600 & -3.11765000 \\
\hline $\mathrm{C}$ & 11.72968200 & 4.76093000 & -1.03962100 \\
\hline $\mathrm{H}$ & 12.25720800 & 3.82910200 & -1.20493900 \\
\hline $\mathrm{C}$ & 11.41258800 & 5.15309300 & 0.25359500 \\
\hline $\mathrm{H}$ & 11.62334400 & 4.51174100 & 1.10063000 \\
\hline $\mathrm{O}$ & 9.21100000 & 5.80139000 & 2.44536400 \\
\hline $\mathrm{O}$ & 5.91624800 & 3.01663200 & 3.31814400 \\
\hline $\mathrm{Fe}$ & 7.36155300 & 3.64632900 & 3.11266800 \\
\hline $\mathrm{N}$ & 8.01761800 & 3.47238900 & 5.00627700 \\
\hline $\mathrm{N}$ & 6.66262200 & 5.35538500 & 3.78422300 \\
\hline $\mathrm{N}$ & 7.26862500 & 4.17014600 & 1.24895900 \\
\hline $\mathrm{N}$ & 7.96068100 & 1.92607200 & 2.37227300 \\
\hline $\mathrm{C}$ & 8.85964400 & 2.61577600 & 5.57244300 \\
\hline $\mathrm{H}$ & 9.41710400 & 1.97037600 & 4.90408400 \\
\hline $\mathrm{C}$ & 9.02148200 & 2.53831500 & 6.96265600 \\
\hline $\mathrm{H}$ & 9.71103800 & 1.81241100 & 7.37673800 \\
\hline $\mathrm{C}$ & 8.27342900 & 3.35870100 & 7.78145200 \\
\hline $\mathrm{H}$ & 8.35134800 & 3.28073500 & 8.86164900 \\
\hline $\mathrm{C}$ & 7.40285200 & 4.30668500 & 7.20328400 \\
\hline $\mathrm{C}$ & 7.34546000 & 4.34025000 & 5.80334600 \\
\hline $\mathrm{C}$ & 6.57594300 & 5.21639000 & 7.94792500 \\
\hline $\mathrm{H}$ & 6.58894700 & 5.16485700 & 9.03181500 \\
\hline $\mathrm{C}$ & 5.76534300 & 6.10311400 & 7.31000100 \\
\hline $\mathrm{H}$ & 5.11818500 & 6.76274000 & 7.87907300 \\
\hline $\mathrm{C}$ & 5.75171200 & 6.18954000 & 5.87710300 \\
\hline $\mathrm{C}$ & 6.56095900 & 5.32618100 & 5.13756000 \\
\hline $\mathrm{C}$ & 4.95796300 & 7.08097600 & 5.12426200 \\
\hline $\mathrm{H}$ & 4.24389900 & 7.72918900 & 5.62308200 \\
\hline $\mathrm{C}$ & 5.07726500 & 7.11101300 & 3.75808600 \\
\hline $\mathrm{H}$ & 4.42861500 & 7.76887500 & 3.19754800 \\
\hline $\mathrm{C}$ & 5.98454500 & 6.25365000 & 3.07774000 \\
\hline $\mathrm{C}$ & 6.19237200 & 6.36523600 & 1.61413200 \\
\hline
\end{tabular}




$\begin{array}{lccc}\mathrm{C} & 5.78490600 & 7.54613600 & 1.01314700 \\ \mathrm{H} & 5.35706800 & 8.33411700 & 1.61906000 \\ \mathrm{C} & 5.91998500 & 7.81144700 & -0.35894600 \\ \mathrm{H} & 5.54734500 & 8.74522300 & -0.76288500 \\ \mathrm{C} & 6.53148800 & 6.88960100 & -1.15812700 \\ \mathrm{H} & 6.65853100 & 7.06341300 & -2.22189700 \\ \mathrm{C} & 7.00836400 & 5.67959700 & -0.60012200 \\ \mathrm{C} & 6.83007500 & 5.38283800 & 0.78238100 \\ \mathrm{C} & 7.68145400 & 4.75148500 & -1.42294900 \\ \mathrm{H} & 7.83093000 & 4.98761200 & -2.47187600 \\ \mathrm{C} & 8.12369600 & 3.56465800 & -0.91078500 \\ \mathrm{H} & 8.63664600 & 2.84624400 & -1.53559400 \\ \mathrm{C} & 7.86762900 & 3.28818400 & 0.43986400 \\ \mathrm{C} & 8.18582100 & 1.98088800 & 1.04774600 \\ \mathrm{C} & 8.57722700 & 0.85536100 & 0.33617700 \\ \mathrm{H} & 8.74604200 & 0.89648100 & -0.73224900 \\ \mathrm{C} & 8.70734800 & -0.35615400 & 1.01082200 \\ \mathrm{H} & 9.00313300 & -1.25033600 & 0.47337100 \\ \mathrm{C} & 8.40898700 & -0.41128900 & 2.36570800 \\ \mathrm{H} & 8.44787100 & -1.34379000 & 2.91579800 \\ \mathrm{C} & 8.02764900 & 0.75754900 & 3.01197000 \\ \mathrm{H} & 7.73703900 & 0.75571000 & 4.05527000 \\ \mathrm{O} & 10.33014900 & 3.40517400 & 2.82044700 \\ \mathrm{H} & 10.97016500 & 3.42168100 & 3.53734600 \\ \mathrm{H} & 10.03714500 & 4.33265800 & 2.71158000 \\ & & & \end{array}$

Int1 1

35

$\begin{array}{lccc}\mathrm{Fe} & 10.65796300 & 7.30228600 & 1.89248800 \\ \mathrm{Cl} & 12.10525300 & 9.04819500 & 2.15506000 \\ \mathrm{~N} & 12.03038900 & 6.28743200 & 2.96981700 \\ \mathrm{C} & 13.26236000 & 5.89845100 & 2.68010000 \\ \mathrm{H} & 13.66604100 & 6.21405800 & 1.72610300 \\ \mathrm{~N} & 9.86426300 & 7.60642700 & 3.66496600 \\ \mathrm{C} & 14.03889000 & 5.14266200 & 3.57388400 \\ \mathrm{H} & 15.04024300 & 4.84821300 & 3.28362100 \\ \mathrm{~N} & 9.52063700 & 8.63124100 & 0.99322200 \\ \mathrm{C} & 13.51779400 & 4.79085800 & 4.79843700 \\ \mathrm{H} & 14.09338400 & 4.18912600 & 5.49476700 \\ \mathrm{~N} & 11.30646200 & 6.96250400 & 0.05241300 \\ \mathrm{C} & 12.24146900 & 5.27195600 & 5.16404300 \\ \mathrm{C} & 11.56255500 & 6.05553300 & 4.21961600 \\ \mathrm{C} & 11.62128000 & 5.04105500 & 6.44015800 \\ \mathrm{H} & 12.13143900 & 4.40749500 & 7.15841600 \\ \mathrm{C} & 10.43856900 & 5.63309800 & 6.75935700 \\ \mathrm{H} & 9.98720400 & 5.47295700 & 7.73317300\end{array}$




\begin{tabular}{|c|c|c|c|}
\hline $\mathrm{C}$ & 9.80019400 & 6.53757100 & 5.84577400 \\
\hline $\mathrm{C}$ & 10.36140700 & 6.73742900 & 4.58129800 \\
\hline $\mathrm{C}$ & 8.62822200 & 7.27070200 & 6.12283600 \\
\hline $\mathrm{H}$ & 8.11213000 & 7.13748700 & 7.06929700 \\
\hline $\mathrm{C}$ & 8.14989300 & 8.15729900 & 5.19529900 \\
\hline $\mathrm{H}$ & 7.23912000 & 8.69531700 & 5.41295600 \\
\hline $\mathrm{C}$ & 8.81260400 & 8.36102900 & 3.95319200 \\
\hline $\mathrm{C}$ & 8.35171300 & 9.41723800 & 3.02122200 \\
\hline $\mathrm{C}$ & 7.57369300 & 10.42818200 & 3.56761300 \\
\hline $\mathrm{H}$ & 7.38945700 & 10.44257900 & 4.63380300 \\
\hline $\mathrm{C}$ & 7.03502400 & 11.48975100 & 2.82289100 \\
\hline $\mathrm{H}$ & 6.43813300 & 12.24662200 & 3.31800500 \\
\hline $\mathrm{C}$ & 7.29804600 & 11.56153300 & 1.48504000 \\
\hline $\mathrm{H}$ & 6.90575000 & 12.37155200 & 0.87866100 \\
\hline $\mathrm{C}$ & 8.13358900 & 10.59401100 & 0.87710100 \\
\hline $\mathrm{C}$ & 8.68434800 & 9.51113200 & 1.62606800 \\
\hline $\mathrm{C}$ & 8.45968000 & 10.72333400 & -0.48981000 \\
\hline $\mathrm{H}$ & 8.05453400 & 11.55662000 & -1.05533900 \\
\hline $\mathrm{C}$ & 9.30030700 & 9.82523200 & -1.08005500 \\
\hline $\mathrm{H}$ & 9.58368100 & 9.93833800 & -2.11739100 \\
\hline $\mathrm{C}$ & 9.83048800 & 8.78917700 & -0.29482500 \\
\hline $\mathrm{C}$ & 10.78919500 & 7.81504800 & -0.84989500 \\
\hline $\mathrm{C}$ & 11.12184600 & 7.72665700 & -2.19749900 \\
\hline $\mathrm{H}$ & 10.71912800 & 8.42407200 & -2.92032800 \\
\hline $\mathrm{C}$ & 11.98598400 & 6.72191600 & -2.61822700 \\
\hline $\mathrm{H}$ & 12.27181600 & 6.64875500 & -3.66172200 \\
\hline $\mathrm{C}$ & 12.45441300 & 5.80443400 & -1.68661300 \\
\hline $\mathrm{H}$ & 13.10168100 & 4.98497700 & -1.97508400 \\
\hline $\mathrm{C}$ & 12.07814100 & 5.95502400 & -0.35945200 \\
\hline $\mathrm{H}$ & 12.38643100 & 5.24131700 & 0.39241500 \\
\hline $\mathrm{O}$ & 9.66262100 & 6.00742400 & 1.73886100 \\
\hline $\mathrm{O}$ & 5.64557100 & 2.92815900 & 3.56446300 \\
\hline $\mathrm{Fe}$ & 7.16437500 & 3.45147300 & 3.45962400 \\
\hline $\mathrm{N}$ & 7.56314300 & 3.26396800 & 5.44500700 \\
\hline $\mathrm{N}$ & 6.49846400 & 5.22223200 & 4.03489100 \\
\hline $\mathrm{N}$ & 7.16009500 & 3.91316300 & 1.55639900 \\
\hline $\mathrm{N}$ & 7.66537900 & 1.65919700 & 2.79474100 \\
\hline $\mathrm{C}$ & 8.28747100 & 2.38500200 & 6.12713700 \\
\hline $\mathrm{H}$ & 8.87656700 & 1.68100300 & 5.54997200 \\
\hline $\mathrm{C}$ & 8.30007300 & 2.34870200 & 7.52945000 \\
\hline $\mathrm{H}$ & 8.89860400 & 1.60089800 & 8.03590900 \\
\hline $\mathrm{C}$ & 7.52257400 & 3.24058000 & 8.23711700 \\
\hline $\mathrm{H}$ & 7.48186700 & 3.20069800 & 9.32144700 \\
\hline $\mathrm{C}$ & 6.78062700 & 4.21753000 & 7.53832200 \\
\hline $\mathrm{C}$ & 6.86883500 & 4.20196600 & 6.13811700 \\
\hline $\mathrm{C}$ & 5.94977200 & 5.20693200 & 8.16850100 \\
\hline
\end{tabular}




$\begin{array}{lrrr}\mathrm{H} & 5.85432100 & 5.19619700 & 9.24928700 \\ \mathrm{C} & 5.27250300 & 6.12274900 & 7.42539500 \\ \mathrm{H} & 4.62173500 & 6.84617400 & 7.90560300 \\ \mathrm{C} & 5.41195800 & 6.15952100 & 5.99689100 \\ \mathrm{C} & 6.23377600 & 5.22134300 & 5.36692800 \\ \mathrm{C} & 4.76350500 & 7.08096400 & 5.14710700 \\ \mathrm{H} & 4.04295800 & 7.78334800 & 5.55504000 \\ \mathrm{C} & 5.03445900 & 7.07157400 & 3.80241800 \\ \mathrm{H} & 4.49658100 & 7.74989300 & 3.15466400 \\ \mathrm{C} & 5.96123200 & 6.14816400 & 3.24719800 \\ \mathrm{C} & 6.33212200 & 6.22330300 & 1.81392500 \\ \mathrm{C} & 6.14798300 & 7.44510300 & 1.18801700 \\ \mathrm{H} & 5.79428000 & 8.29061800 & 1.76515300 \\ \mathrm{C} & 6.41700100 & 7.67008900 & -0.17240500 \\ \mathrm{H} & 6.22538500 & 8.64638500 & -0.60145600 \\ \mathrm{C} & 6.90957400 & 6.64963700 & -0.93227800 \\ \mathrm{H} & 7.11297000 & 6.78311500 & -1.98998600 \\ \mathrm{C} & 7.15868900 & 5.38709500 & -0.34301600 \\ \mathrm{C} & 6.88353700 & 5.14912700 & 1.03504900 \\ \mathrm{C} & 7.69755400 & 4.34570300 & -1.12540800 \\ \mathrm{H} & 7.90228700 & 4.52437800 & -2.17630200 \\ \mathrm{C} & 7.95466700 & 3.12559700 & -0.56749300 \\ \mathrm{H} & 8.37100700 & 2.32337900 & -1.16124600 \\ \mathrm{C} & 7.66891000 & 2.94026800 & 0.79328100 \\ \mathrm{C} & 7.87575200 & 1.64016400 & 1.46468100 \\ \mathrm{C} & 8.15984600 & 0.45496600 & 0.79730300 \\ \mathrm{H} & 8.31882500 & 0.43863800 & -0.27309900 \\ \mathrm{C} & 8.19464200 & -0.73597500 & 1.51699900 \\ \mathrm{H} & 8.40672200 & -1.67217900 & 1.01259400 \\ \mathrm{C} & 7.90908900 & -0.71153700 & 2.87548000 \\ \mathrm{H} & 7.87452600 & -1.62082800 & 3.46337200 \\ \mathrm{C} & 7.63752100 & 0.51076600 & 3.47557800 \\ \mathrm{H} & 7.35216500 & 0.57029300 & 4.51792500 \\ \mathrm{O} & 9.16019000 & 4.01997200 & 3.33153500 \\ \mathrm{H} & 9.55201900 & 4.23232800 & 4.18409400 \\ \mathrm{H} & 9.36834900 & 4.78032300 & 2.71547400 \\ & & & \end{array}$

Complex 4

34

$\begin{array}{llll}\mathrm{O} & 5.64954800 & 2.88453300 & 3.50641800 \\ \mathrm{Fe} & 7.16391000 & 3.42289000 & 3.45028300 \\ \mathrm{~N} & 7.50202100 & 3.22401300 & 5.44893900 \\ \mathrm{~N} & 6.45496800 & 5.18769000 & 4.03110200 \\ \mathrm{~N} & 7.16503100 & 3.90788600 & 1.54583000 \\ \mathrm{~N} & 7.70937900 & 1.65371800 & 2.76452400 \\ \mathrm{C} & 8.21266600 & 2.34028800 & 6.15308600\end{array}$




\begin{tabular}{|c|c|c|c|}
\hline $\mathrm{H}$ & 8.81958800 & 1.63831700 & 5.59380300 \\
\hline $\mathrm{C}$ & 8.19968100 & 2.30512100 & 7.55172700 \\
\hline $\mathrm{H}$ & 8.79064400 & 1.55919200 & 8.07058900 \\
\hline $\mathrm{C}$ & 7.42354300 & 3.21377600 & 8.24816300 \\
\hline $\mathrm{H}$ & 7.37611300 & 3.18741800 & 9.33275200 \\
\hline $\mathrm{C}$ & 6.71374400 & 4.19305100 & 7.52567600 \\
\hline $\mathrm{C}$ & 6.81140600 & 4.16405300 & 6.12940600 \\
\hline $\mathrm{C}$ & 5.90757600 & 5.21687500 & 8.14045600 \\
\hline $\mathrm{H}$ & 5.80618300 & 5.22035500 & 9.22184800 \\
\hline $\mathrm{C}$ & 5.26666600 & 6.17584500 & 7.38961500 \\
\hline $\mathrm{H}$ & 4.65425700 & 6.92781100 & 7.87628600 \\
\hline $\mathrm{C}$ & 5.40981000 & 6.20410800 & 5.98475900 \\
\hline $\mathrm{C}$ & 6.20488000 & 5.20818600 & 5.34120400 \\
\hline $\mathrm{C}$ & 4.79334300 & 7.15563800 & 5.13546500 \\
\hline $\mathrm{H}$ & 4.11313600 & 7.89405400 & 5.54812400 \\
\hline $\mathrm{C}$ & 5.05654300 & 7.12860000 & 3.79614200 \\
\hline $\mathrm{H}$ & 4.54374200 & 7.83467100 & 3.15863000 \\
\hline $\mathrm{C}$ & 5.94747100 & 6.15915700 & 3.22658000 \\
\hline $\mathrm{C}$ & 6.30975200 & 6.21937600 & 1.83223500 \\
\hline $\mathrm{C}$ & 6.10376500 & 7.46675700 & 1.18196500 \\
\hline $\mathrm{H}$ & 5.76178100 & 8.31349500 & 1.76155900 \\
\hline $\mathrm{C}$ & 6.36497500 & 7.69194700 & -0.15768400 \\
\hline $\mathrm{H}$ & 6.19576200 & 8.67083300 & -0.59133700 \\
\hline $\mathrm{C}$ & 6.84337600 & 6.64640000 & -0.92721400 \\
\hline $\mathrm{H}$ & 7.03618000 & 6.78702100 & -1.98726800 \\
\hline $\mathrm{C}$ & 7.10701100 & 5.37755500 & -0.34655400 \\
\hline $\mathrm{C}$ & 6.87698200 & 5.13389300 & 1.03652600 \\
\hline $\mathrm{C}$ & 7.61845100 & 4.34543000 & -1.15771900 \\
\hline $\mathrm{H}$ & 7.79503100 & 4.52852000 & -2.21336100 \\
\hline $\mathrm{C}$ & 7.89404200 & 3.11960700 & -0.60832100 \\
\hline $\mathrm{H}$ & 8.29642700 & 2.32415100 & -1.22121900 \\
\hline $\mathrm{C}$ & 7.65330300 & 2.92933000 & 0.75677000 \\
\hline $\mathrm{C}$ & 7.89461500 & 1.63603100 & 1.42453000 \\
\hline $\mathrm{C}$ & 8.19273600 & 0.45912900 & 0.75008600 \\
\hline $\mathrm{H}$ & 8.33967800 & 0.44585500 & -0.32238600 \\
\hline $\mathrm{C}$ & 8.26427100 & -0.73474700 & 1.46641200 \\
\hline $\mathrm{H}$ & 8.49146400 & -1.66474300 & 0.95643500 \\
\hline $\mathrm{C}$ & 7.99594500 & -0.71743700 & 2.82793600 \\
\hline $\mathrm{H}$ & 7.98777900 & -1.62853000 & 3.41478200 \\
\hline $\mathrm{C}$ & 7.71122400 & 0.49864600 & 3.43838200 \\
\hline $\mathrm{H}$ & 7.43895700 & 0.54101500 & 4.48435000 \\
\hline $\mathrm{O}$ & 9.16660900 & 4.15730900 & 3.37845800 \\
\hline $\mathrm{H}$ & 9.56312700 & 4.63184800 & 4.11722600 \\
\hline $\mathrm{H}$ & 9.88259300 & 3.69492200 & 2.92839300 \\
\hline
\end{tabular}

\section{TS14}




\begin{tabular}{|c|c|c|c|}
\hline $\mathrm{O}$ & 5.64989400 & 2.90982000 & 3.60927600 \\
\hline $\mathrm{Fe}$ & 7.15996800 & 3.49799100 & 3.47005200 \\
\hline $\mathrm{N}$ & 7.53568800 & 3.26038500 & 5.44740400 \\
\hline $\mathrm{N}$ & 6.51613700 & 5.26235300 & 4.05427300 \\
\hline $\mathrm{N}$ & 7.17056600 & 3.98398700 & 1.57210400 \\
\hline $\mathrm{N}$ & 7.68146800 & 1.71030900 & 2.78561200 \\
\hline $\mathrm{C}$ & 8.23529500 & 2.35453000 & 6.12378700 \\
\hline $\mathrm{H}$ & 8.82599500 & 1.65382800 & 5.54508500 \\
\hline $\mathrm{C}$ & 8.23033700 & 2.29417900 & 7.52370000 \\
\hline $\mathrm{H}$ & 8.81693100 & 1.53286200 & 8.02347300 \\
\hline $\mathrm{C}$ & 7.46574600 & 3.19245800 & 8.23727000 \\
\hline $\mathrm{H}$ & 7.42191200 & 3.14342800 & 9.32080800 \\
\hline $\mathrm{C}$ & 6.75495800 & 4.19840700 & 7.54910000 \\
\hline $\mathrm{C}$ & 6.85415400 & 4.20369600 & 6.14917700 \\
\hline $\mathrm{C}$ & 5.96223200 & 5.20849600 & 8.19187700 \\
\hline $\mathrm{H}$ & 5.86776800 & 5.18734300 & 9.27223300 \\
\hline $\mathrm{C}$ & 5.33965800 & 6.17090900 & 7.46243600 \\
\hline $\mathrm{H}$ & 4.73224100 & 6.92545600 & 7.95072100 \\
\hline $\mathrm{C}$ & 5.48852300 & 6.22481800 & 6.03633000 \\
\hline $\mathrm{C}$ & 6.26072000 & 5.25611300 & 5.39142600 \\
\hline $\mathrm{C}$ & 4.88380500 & 7.18991900 & 5.20298700 \\
\hline $\mathrm{H}$ & 4.21349000 & 7.93094100 & 5.62682700 \\
\hline $\mathrm{C}$ & 5.13463300 & 7.17819900 & 3.85523600 \\
\hline $\mathrm{H}$ & 4.62924700 & 7.89385600 & 3.22231700 \\
\hline $\mathrm{C}$ & 6.01188100 & 6.22183300 & 3.28137700 \\
\hline $\mathrm{C}$ & 6.38046900 & 6.30744000 & 1.84851400 \\
\hline $\mathrm{C}$ & 6.23645000 & 7.54403600 & 1.23601300 \\
\hline $\mathrm{H}$ & 5.92527400 & 8.39611500 & 1.82685200 \\
\hline $\mathrm{C}$ & 6.49302300 & 7.77436000 & -0.12565900 \\
\hline $\mathrm{H}$ & 6.34735500 & 8.76522900 & -0.53902200 \\
\hline $\mathrm{C}$ & 6.91946600 & 6.73898000 & -0.90675200 \\
\hline $\mathrm{H}$ & 7.11372700 & 6.87619300 & -1.96538000 \\
\hline $\mathrm{C}$ & 7.14003200 & 5.46456900 & -0.33031500 \\
\hline $\mathrm{C}$ & 6.89339800 & 5.22711700 & 1.05527300 \\
\hline $\mathrm{C}$ & 7.63796100 & 4.41398900 & -1.12824100 \\
\hline $\mathrm{H}$ & 7.82195000 & 4.59216300 & -2.18298500 \\
\hline $\mathrm{C}$ & 7.89668800 & 3.19059800 & -0.57620300 \\
\hline $\mathrm{H}$ & 8.29074500 & 2.38700100 & -1.18250300 \\
\hline $\mathrm{C}$ & 7.65047300 & 3.00699700 & 0.79110700 \\
\hline $\mathrm{C}$ & 7.86762700 & 1.70406600 & 1.45148800 \\
\hline $\mathrm{C}$ & 8.14034500 & 0.52660500 & 0.76694800 \\
\hline $\mathrm{H}$ & 8.28659100 & 0.52365100 & -0.30497100 \\
\hline $\mathrm{C}$ & 8.18221600 & -0.67259300 & 1.47080600 \\
\hline $\mathrm{H}$ & 8.38873700 & -1.60218500 & 0.95233200 \\
\hline$C$ & 7.91032500 & -0.66391300 & 2.83192100 \\
\hline
\end{tabular}




$\begin{array}{llrl}\mathrm{H} & 7.87850600 & -1.57940300 & 3.40995500 \\ \mathrm{C} & 7.65211500 & 0.55131500 & 3.44977400 \\ \mathrm{H} & 7.37689400 & 0.59493000 & 4.49492300 \\ \mathrm{O} & 9.17871900 & 4.12409900 & 3.38474900 \\ \mathrm{H} & 9.40257100 & 4.87758100 & 2.82758800 \\ \mathrm{H} & 9.64345600 & 4.24263600 & 4.22033400 \\ \mathrm{O} & 4.30509600 & 3.81856700 & 2.51402000 \\ \mathrm{~N} & 4.13862000 & 3.16333300 & 1.35138500 \\ \mathrm{O} & 3.35561900 & 3.76202900 & 0.65229000 \\ \mathrm{O} & 4.74452900 & 2.14735700 & 1.13685400\end{array}$

\section{Int14}

22

$\mathrm{O}$

$\mathrm{Fe}$

$\mathrm{N}$

$\mathrm{N}$

$\mathrm{N}$

$\mathrm{N}$

C

$\mathrm{H}$

C

$\mathrm{H}$

C

$\mathrm{H}$

$\mathrm{C}$

C

C

$\mathrm{H}$

C

$\mathrm{H}$

C

C

C

$\mathrm{H}$

C

$\mathrm{H}$

C

C

C

$\mathrm{H}$

C

$\mathrm{H}$

C

$\mathrm{H}$

C

\subsection{0}

7.24722300

7.58129100

6.55475700

7.19545400

7.73016600

8.27136700

8.87932600

8.23450600

8.81473800

7.44615500

7.37716400

6.74210200

6.87347400

5.92342300

5.80697500

5.30293000

4.67502400

5.47948500

6.27983300

4.87325200

4.18656000

5.14045600

4.63211200

6.03813900

6.41176300

6.27057800

5.95957600

6.53086900

6.38841500

6.95769300

7.15539500

7.17460600
2.86071000

3.52131700

3.25867000

5.26569300

3.98942200

1.72120100

2.35515400

1.66027800

2.28446600

1.52444100

3.17255000

3.11758900

4.17564500

4.19133900

5.17032600

5.13880100

6.12947600

6.87203300

6.19464400

5.24325800

7.15637800

7.88450900

7.15738300

7.86838300

6.21774900

6.30987700

7.54824100

8.39934300

7.77991300

$8.77206800-0.54350300$

$6.74604200-0.91564800$

$6.88709700-1.97316200$

$5.46959900-0.34249000$

3.64969600

3.46689300

5.45186600

4.04647300

1.55973400

2.79097200

6.14083700

5.57219600

7.53958000

8.04863800

8.23938300

9.32133000

7.54019600

6.14270700

8.17448100

9.25243900

7.43997400

7.92064600

6.01750800

5.37995700

5.18185800

5.60192100

3.83673000

3.20071200

3.27053500

1.83834700

1.22924400

1.82204300

$-0.13199200$

.

(n)

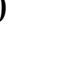




$\begin{array}{lrrr}\mathrm{C} & 6.92262000 & 5.23090800 & 1.04124100 \\ \mathrm{C} & 7.67798900 & 4.41781200 & -1.13636000 \\ \mathrm{H} & 7.86957500 & 4.59556300 & -2.18987500 \\ \mathrm{C} & 7.93587300 & 3.19470400 & -0.58187900 \\ \mathrm{H} & 8.33858500 & 2.39272800 & -1.18492500 \\ \mathrm{C} & 7.68316200 & 3.01216900 & 0.78506600 \\ \mathrm{C} & 7.90069600 & 1.71004900 & 1.45290400 \\ \mathrm{C} & 8.14622000 & 0.52768600 & 0.76770000 \\ \mathrm{H} & 8.27863200 & 0.52071400 & -0.30607600 \\ \mathrm{C} & 8.17378600 & -0.67170500 & 1.47291900 \\ \mathrm{H} & 8.35898600 & -1.60523300 & 0.95339000 \\ \mathrm{C} & 7.91378400 & -0.65746700 & 2.83606800 \\ \mathrm{H} & 7.87044800 & -1.57198300 & 3.41483200 \\ \mathrm{C} & 7.68533400 & 0.56309400 & 3.45537800 \\ \mathrm{H} & 7.42318900 & 0.61293700 & 4.50356000 \\ \mathrm{O} & 9.22090900 & 4.16646300 & 3.34811200 \\ \mathrm{H} & 9.39478100 & 5.02203800 & 2.93991700 \\ \mathrm{H} & 9.75833400 & 4.11571600 & 4.14641200 \\ \mathrm{O} & 4.63979100 & 3.58823000 & 2.98825700 \\ \mathrm{~N} & 4.29395500 & 2.92649000 & 1.71965300 \\ \mathrm{O} & 3.47034000 & 3.56090200 & 1.14064300 \\ \mathrm{O} & 4.86828600 & 1.91144100 & 1.46647000\end{array}$

TS24

26

$\begin{array}{llll}\mathrm{O} & 5.37113000 & 2.50046400 & 3.62672100 \\ \mathrm{Fe} & 7.72391400 & 3.57843200 & 3.43478700 \\ \mathrm{~N} & 7.74085000 & 3.38856900 & 5.57960100 \\ \mathrm{~N} & 6.62849000 & 5.28905300 & 3.97341200 \\ \mathrm{~N} & 7.34799000 & 4.07405400 & 1.37786600 \\ \mathrm{~N} & 7.96675000 & 1.69884300 & 2.49941800 \\ \mathrm{C} & 8.35984400 & 2.52156000 & 6.37169400 \\ \mathrm{H} & 9.10693500 & 1.88442700 & 5.90653300 \\ \mathrm{C} & 8.08770500 & 2.41320400 & 7.74221200 \\ \mathrm{H} & 8.62510300 & 1.68852700 & 8.34183500 \\ \mathrm{C} & 7.13005400 & 3.23347700 & 8.29493900 \\ \mathrm{H} & 6.88545900 & 3.16611600 & 9.35047900 \\ \mathrm{C} & 6.46389200 & 4.17668400 & 7.48325400 \\ \mathrm{C} & 6.81954700 & 4.22757200 & 6.12307500 \\ \mathrm{C} & 5.45499900 & 5.06449600 & 7.98815800 \\ \mathrm{H} & 5.18816700 & 5.00490400 & 9.03800700 \\ \mathrm{C} & 4.84424100 & 5.95665400 & 7.16931800 \\ \mathrm{H} & 4.07634400 & 6.62149300 & 7.55061500 \\ \mathrm{C} & 5.21157900 & 6.05222200 & 5.78594200 \\ \mathrm{C} & 6.20313200 & 5.20968000 & 5.26320700 \\ \mathrm{C} & 4.61227300 & 6.95735000 & 4.88484400\end{array}$




$\begin{array}{lccc}\mathrm{H} & 3.80303600 & 7.59721400 & 5.22304200 \\ \mathrm{C} & 5.04782900 & 7.02272200 & 3.58550000 \\ \mathrm{H} & 4.56380200 & 7.69482800 & 2.88901300 \\ \mathrm{C} & 6.11162400 & 6.19730900 & 3.14586300 \\ \mathrm{C} & 6.65338400 & 6.38615200 & 1.77105100 \\ \mathrm{C} & 6.61605400 & 7.67262900 & 1.25970600 \\ \mathrm{H} & 6.29735600 & 8.48793700 & 1.89974900 \\ \mathrm{C} & 6.98433500 & 7.98782500 & -0.06293000 \\ \mathrm{H} & 6.93634700 & 9.01701400 & -0.39901400 \\ \mathrm{C} & 7.37002800 & 6.98978200 & -0.91328100 \\ \mathrm{H} & 7.62056900 & 7.20025300 & -1.94796800 \\ \mathrm{C} & 7.46064400 & 5.65792600 & -0.43916500 \\ \mathrm{C} & 7.15759400 & 5.34572200 & 0.91758600 \\ \mathrm{C} & 7.86783200 & 4.60798900 & -1.29188100 \\ \mathrm{H} & 8.09932000 & 4.82846300 & -2.32937000 \\ \mathrm{C} & 7.97484500 & 3.32895700 & -0.81460500 \\ \mathrm{H} & 8.29796700 & 2.52677700 & -1.46505600 \\ \mathrm{C} & 7.71631600 & 3.09951000 & 0.55006000 \\ \mathrm{C} & 7.87219300 & 1.74508800 & 1.15393800 \\ \mathrm{C} & 7.88952500 & 0.58010400 & 0.39433100 \\ \mathrm{H} & 7.78253100 & 0.61644800 & -0.68238800 \\ \mathrm{C} & 8.01638300 & -0.64876100 & 1.03544700 \\ \mathrm{H} & 8.02860800 & -1.56541000 & 0.45602200 \\ \mathrm{C} & 8.11407700 & -0.68622500 & 2.42077800 \\ \mathrm{H} & 8.20752900 & -1.62232400 & 2.95759800 \\ \mathrm{C} & 8.07804200 & 0.51484400 & 3.11362900 \\ \mathrm{H} & 8.12753400 & 0.53623800 & 4.19708400 \\ \mathrm{O} & 9.76083500 & 4.25401100 & 3.40653800 \\ \mathrm{H} & 10.26417900 & 4.52605400 & 2.63307700 \\ \mathrm{H} & 10.20635700 & 4.60678500 & 4.18321600 \\ \mathrm{O} & 4.42598100 & 3.20537900 & 3.25910500 \\ \mathrm{~N} & 2.87355300 & 2.03745100 & 3.40976300 \\ \mathrm{O} & 1.86909300 & 2.56691000 & 3.10702500 \\ \mathrm{O} & 3.27357000 & 0.99880500 & 3.78820000 \\ & & & \\ & & & \\ \mathrm{H} & & & \end{array}$

Complex 5

24

$\begin{array}{llll}\mathrm{O} & 5.52477600 & 2.84438200 & 3.49109100 \\ \mathrm{Fe} & 7.05769600 & 3.39948000 & 3.44242600 \\ \mathrm{~N} & 7.39427400 & 3.18382400 & 5.41522900 \\ \mathrm{~N} & 6.32610500 & 5.12782000 & 4.01762400 \\ \mathrm{~N} & 7.07781600 & 3.87877900 & 1.54153000 \\ \mathrm{~N} & 7.60406800 & 1.62967700 & 2.76129500 \\ \mathrm{C} & 8.17615100 & 2.34225900 & 6.08711800 \\ \mathrm{H} & 8.77903300 & 1.66188700 & 5.49877500 \\ \mathrm{C} & 8.25234600 & 2.34458200 & 7.48470800\end{array}$




\begin{tabular}{|c|c|c|c|}
\hline $\mathrm{H}$ & 8.89518600 & 1.62756900 & 7.98069300 \\
\hline $\mathrm{C}$ & 7.51037200 & 3.25852000 & 8.20714700 \\
\hline $\mathrm{H}$ & 7.53940400 & 3.26635300 & 9.29208800 \\
\hline $\mathrm{C}$ & 6.74265500 & 4.21063700 & 7.50879500 \\
\hline $\mathrm{C}$ & 6.74651900 & 4.13678800 & 6.10952500 \\
\hline 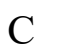 & 5.98115400 & 5.25305400 & 8.13594900 \\
\hline $\mathrm{H}$ & 5.94409600 & 5.28921600 & 9.22035000 \\
\hline $\mathrm{C}$ & 5.30684600 & 6.19706000 & 7.39770400 \\
\hline $\mathrm{H}$ & 4.73310200 & 6.97064800 & 7.89644500 \\
\hline $\mathrm{C}$ & 5.37746600 & 6.19184600 & 5.98564500 \\
\hline $\mathrm{C}$ & 6.11735900 & 5.16934700 & 5.33376700 \\
\hline $\mathrm{C}$ & 4.74985300 & 7.14162300 & 5.14142900 \\
\hline $\mathrm{H}$ & 4.09592000 & 7.89683500 & 5.56473300 \\
\hline $\mathrm{C}$ & 4.97896200 & 7.09844500 & 3.79747900 \\
\hline $\mathrm{H}$ & 4.46805300 & 7.80812100 & 3.16294300 \\
\hline $\mathrm{C}$ & 5.84536900 & 6.11154600 & 3.22103000 \\
\hline $\mathrm{C}$ & 6.23595600 & 6.18253400 & 1.83202500 \\
\hline $\mathrm{C}$ & 6.06663500 & 7.44034500 & 1.20003500 \\
\hline $\mathrm{H}$ & 5.70449600 & 8.27739200 & 1.78043900 \\
\hline $\mathrm{C}$ & 6.39608300 & 7.68900000 & -0.12047400 \\
\hline $\mathrm{H}$ & 6.25524600 & 8.67766900 & -0.54042300 \\
\hline $\mathrm{C}$ & 6.91710900 & 6.66090000 & -0.88439700 \\
\hline $\mathrm{H}$ & 7.17809600 & 6.82618600 & -1.92548500 \\
\hline $\mathrm{C}$ & 7.13986600 & 5.38329600 & -0.32101000 \\
\hline $\mathrm{C}$ & 6.82573800 & 5.11101300 & 1.04071500 \\
\hline $\mathrm{C}$ & 7.70732500 & 4.36711600 & -1.11591500 \\
\hline $\mathrm{H}$ & 7.95661200 & 4.57441900 & -2.15159600 \\
\hline $\mathrm{C}$ & 7.95115700 & 3.13528200 & -0.57218700 \\
\hline $\mathrm{H}$ & 8.40684900 & 2.35394900 & -1.16471100 \\
\hline $\mathrm{C}$ & 7.62538200 & 2.92089400 & 0.77246800 \\
\hline $\mathrm{C}$ & 7.85318800 & 1.62688100 & 1.43772600 \\
\hline $\mathrm{C}$ & 8.21557700 & 0.46325500 & 0.77033100 \\
\hline $\mathrm{H}$ & 8.41422400 & 0.46449800 & -0.29350600 \\
\hline $\mathrm{C}$ & 8.29326100 & -0.72846900 & 1.48440900 \\
\hline $\mathrm{H}$ & 8.57653000 & -1.64742300 & 0.98384900 \\
\hline $\mathrm{C}$ & 7.96535800 & -0.72564200 & 2.83257500 \\
\hline $\mathrm{H}$ & 7.96705700 & -1.63753400 & 3.41730100 \\
\hline $\mathrm{C}$ & 7.61284300 & 0.47753300 & 3.43198900 \\
\hline $\mathrm{H}$ & 7.30068600 & 0.51452600 & 4.46727500 \\
\hline $\mathrm{Cl}$ & 9.23192000 & 4.20078300 & 3.41112900 \\
\hline
\end{tabular}

TS15

12

$\begin{array}{llll}\mathrm{O} & 5.62025200 & 2.87938300 & 3.63667000 \\ \mathrm{Fe} & 7.12414900 & 3.48451900 & 3.47720500 \\ \mathrm{~N} & 7.47092100 & 3.26199300 & 5.44748200\end{array}$




\begin{tabular}{|c|c|c|c|}
\hline $\mathrm{N}$ & 6.41880700 & 5.22292700 & 4.05293400 \\
\hline $\mathrm{N}$ & 7.08152400 & 3.94032200 & 1.56664800 \\
\hline $\mathrm{N}$ & 7.65068900 & 1.69574900 & 2.79221100 \\
\hline $\mathrm{C}$ & 8.24590000 & 2.41896200 & 6.11006100 \\
\hline $\mathrm{H}$ & 8.87747300 & 1.76772200 & 5.51870900 \\
\hline $\mathrm{C}$ & 8.28068800 & 2.38342600 & 7.51202600 \\
\hline $\mathrm{H}$ & 8.92370700 & 1.66538200 & 8.00603900 \\
\hline $\mathrm{C}$ & 7.49709200 & 3.25656200 & 8.23369000 \\
\hline $\mathrm{H}$ & 7.49060400 & 3.23049500 & 9.31873600 \\
\hline $\mathrm{C}$ & 6.72948300 & 4.22012800 & 7.54865400 \\
\hline $\mathrm{C}$ & 6.78322100 & 4.19300900 & 6.14792500 \\
\hline $\mathrm{C}$ & 5.93253700 & 5.23044200 & 8.18750600 \\
\hline $\mathrm{H}$ & 5.86406100 & 5.23024200 & 9.27019900 \\
\hline $\mathrm{C}$ & 5.28361900 & 6.17263100 & 7.45526900 \\
\hline $\mathrm{H}$ & 4.68099400 & 6.93122200 & 7.94349200 \\
\hline $\mathrm{C}$ & 5.40765500 & 6.20941800 & 6.02580000 \\
\hline $\mathrm{C}$ & 6.16751400 & 5.22874700 & 5.38501300 \\
\hline $\mathrm{C}$ & 4.80767200 & 7.17028400 & 5.18706700 \\
\hline $\mathrm{H}$ & 4.14361400 & 7.91952500 & 5.60606200 \\
\hline $\mathrm{C}$ & 5.06252400 & 7.14842100 & 3.84059400 \\
\hline $\mathrm{H}$ & 4.56722400 & 7.86335800 & 3.19891100 \\
\hline $\mathrm{C}$ & 5.93749000 & 6.18309300 & 3.27574300 \\
\hline $\mathrm{C}$ & 6.32658600 & 6.26881600 & 1.84897600 \\
\hline $\mathrm{C}$ & 6.22140300 & 7.51308700 & 1.25075000 \\
\hline $\mathrm{H}$ & 5.92770600 & 8.36547400 & 1.84990400 \\
\hline $\mathrm{C}$ & 6.50248700 & 7.74999700 & -0.10515200 \\
\hline $\mathrm{H}$ & 6.39081200 & 8.74897300 & -0.50958400 \\
\hline $\mathrm{C}$ & 6.91124100 & 6.71209600 & -0.89051000 \\
\hline $\mathrm{H}$ & 7.12464500 & 6.85343100 & -1.94488900 \\
\hline $\mathrm{C}$ & 7.09447800 & 5.42835000 & -0.32436500 \\
\hline $\mathrm{C}$ & 6.82839800 & 5.18458400 & 1.05445600 \\
\hline $\mathrm{C}$ & 7.57898400 & 4.37459700 & -1.12607900 \\
\hline $\mathrm{H}$ & 7.77882300 & 4.55870800 & -2.17663100 \\
\hline $\mathrm{C}$ & 7.80999700 & 3.14724000 & -0.57824900 \\
\hline $\mathrm{H}$ & 8.20085600 & 2.33869500 & -1.17978700 \\
\hline $\mathrm{C}$ & 7.55819200 & 2.96753400 & 0.79040100 \\
\hline $\mathrm{C}$ & 7.79709500 & 1.67433900 & 1.45753600 \\
\hline $\mathrm{C}$ & 8.07352400 & 0.49179200 & 0.78116100 \\
\hline $\mathrm{H}$ & 8.18406700 & 0.47916800 & -0.29479600 \\
\hline $\mathrm{C}$ & 8.16875900 & -0.69208300 & 1.50083700 \\
\hline $\mathrm{H}$ & 8.38144000 & -1.62485500 & 0.99090500 \\
\hline $\mathrm{C}$ & 7.94759400 & -0.66645500 & 2.87099700 \\
\hline $\mathrm{H}$ & 7.96540600 & -1.57121300 & 3.46594500 \\
\hline $\mathrm{C}$ & 7.67668000 & 0.55155900 & 3.47611400 \\
\hline $\mathrm{H}$ & 7.44233400 & 0.61117500 & 4.53014200 \\
\hline $\mathrm{Cl}$ & 9.30421100 & 4.22930000 & 3.40373200 \\
\hline
\end{tabular}




$\begin{array}{llll}\mathrm{O} & 4.24896800 & 3.72982500 & 2.43687300 \\ \mathrm{~N} & 4.16425000 & 3.04352300 & 1.29925900 \\ \mathrm{O} & 3.50643400 & 3.63482700 & 0.46718500 \\ \mathrm{O} & 4.71368900 & 1.97258100 & 1.19437500\end{array}$

Int15

12

$\begin{array}{lrrr}\mathrm{O} & 5.53296700 & 2.88076800 & 3.71050100 \\ \mathrm{Fe} & 7.23371000 & 3.53790300 & 3.45997100 \\ \mathrm{~N} & 7.51845200 & 3.27658500 & 5.43660800 \\ \mathrm{~N} & 6.46794100 & 5.24647900 & 4.03360500 \\ \mathrm{~N} & 7.10236100 & 3.96877700 & 1.53396300 \\ \mathrm{~N} & 7.67085500 & 1.73047600 & 2.77116300 \\ \mathrm{C} & 8.27933700 & 2.43285600 & 6.11464100 \\ \mathrm{H} & 8.92596500 & 1.78574500 & 5.53387200 \\ \mathrm{C} & 8.27803000 & 2.38358300 & 7.51697300 \\ \mathrm{H} & 8.91193500 & 1.66513400 & 8.02214700 \\ \mathrm{C} & 7.46825400 & 3.24404100 & 8.22438500 \\ \mathrm{H} & 7.43192500 & 3.20812800 & 9.30861300 \\ \mathrm{C} & 6.71022300 & 4.20674600 & 7.52720400 \\ \mathrm{C} & 6.80301400 & 4.19420300 & 6.12814100 \\ \mathrm{C} & 5.88413300 & 5.19950600 & 8.15693300 \\ \mathrm{H} & 5.78833600 & 5.18580200 & 9.23751200 \\ \mathrm{C} & 5.24013800 & 6.14021300 & 7.41919900 \\ \mathrm{H} & 4.61410100 & 6.88464500 & 7.89973100 \\ \mathrm{C} & 5.39847800 & 6.19261100 & 5.99344900 \\ \mathrm{C} & 6.19088800 & 5.23227600 & 5.36100100 \\ \mathrm{C} & 4.79812700 & 7.15052900 & 5.15149400 \\ \mathrm{H} & 4.11484400 & 7.88508600 & 5.56579200 \\ \mathrm{C} & 5.07280700 & 7.14376600 & 3.80820900 \\ \mathrm{H} & 4.57555800 & 7.85442800 & 3.16289500 \\ \mathrm{C} & 5.97278400 & 6.19819300 & 3.25183300 \\ \mathrm{C} & 6.36938900 & 6.29519500 & 1.82635100 \\ \mathrm{C} & 6.27562200 & 7.54488100 & 1.23841600 \\ \mathrm{H} & 5.98720900 & 8.39519700 & 1.84366400 \\ \mathrm{C} & 6.56478000 & 7.78794100 & -0.11564300 \\ \mathrm{H} & 6.46471100 & 8.79121100 & -0.51267000 \\ \mathrm{C} & 6.96814800 & 6.75341300 & -0.90916600 \\ \mathrm{H} & 7.18860600 & 6.90378100 & -1.96092500 \\ \mathrm{C} & 7.13741700 & 5.46301200 & -0.35352100 \\ \mathrm{C} & 6.86261900 & 5.21298000 & 1.02204300 \\ \mathrm{C} & 7.62024000 & 4.40740200 & -1.15520600 \\ \mathrm{H} & 7.82989900 & 4.59339400 & -2.20363100 \\ \mathrm{C} & 7.84136000 & 3.17662500 & -0.60887900 \\ \mathrm{H} & 8.23589200 & 2.36862700 & -1.20939700 \\ & 7.58098100 & 2.99659000 & 0.75949100\end{array}$




$\begin{array}{lrrr}\mathrm{C} & 7.81135400 & 1.70228000 & 1.43405600 \\ \mathrm{C} & 8.05930000 & 0.51252200 & 0.76100500 \\ \mathrm{H} & 8.16729800 & 0.49447500 & -0.31532700 \\ \mathrm{C} & 8.12412300 & -0.67201700 & 1.48433000 \\ \mathrm{H} & 8.31270200 & -1.61113900 & 0.97641200 \\ \mathrm{C} & 7.90329300 & -0.63862900 & 2.85417800 \\ \mathrm{H} & 7.89765100 & -1.54276200 & 3.45024700 \\ \mathrm{C} & 7.66855300 & 0.58790700 & 3.45759600 \\ \mathrm{H} & 7.44355500 & 0.65826900 & 4.51304100 \\ \mathrm{Cl} & 9.36979500 & 4.26953100 & 3.34788300 \\ \mathrm{O} & 4.59760300 & 3.48483100 & 2.88984100 \\ \mathrm{~N} & 4.31464800 & 2.67384100 & 1.74006800 \\ \mathrm{O} & 3.53281200 & 3.22098200 & 1.01481800 \\ \mathrm{O} & 4.86488000 & 1.61309300 & 1.65133600\end{array}$

\section{TS25}

16

$\mathrm{O}$

$\mathrm{Fe}$

$\mathrm{N}$

$\mathrm{N}$

$\mathrm{N}$

$\mathrm{N}$

C

$\mathrm{H}$

C

$\mathrm{H}$

C

$\mathrm{H}$

C

C

C

$\mathrm{H}$

C

$\mathrm{H}$

C

C

C

$\mathrm{H}$

C

$\mathrm{H}$

C

C

C

$\mathrm{H}$

C

\subsection{0}

7.60298000

7.55174300

6.53897300

7.19340600

7.73954500

8.18124600

8.88361200

7.97312400

8.51425200

7.07356800

6.87669700

6.40999000

6.71058400

5.45867600

5.22289300

4.86278000

4.13704800

5.19379600

6.12620900

4.62249300

3.84795400

5.05306500

4.60670100

6.07711800

6.66981700

6.75623700

6.46405700

7.23298700
2.54707800

3.53277600

3.47953100

5.34961900

4.07601600

1.63265500

2.63651200

1.95670600

2.62290600

1.91566200

3.50960200

3.51135000

4.43462100

4.39158300

5.39647700

5.40900700

6.26840100

6.98777500

6.27804400

5.35948000

7.16671100

7.85824800

7.15570300

7.82464600

6.26388700

6.42641700

7.72508400

8.54203500

$8.04836800-0.05246200$
3.57453500

3.38267400

5.56018500

3.90490500

1.28759500

2.34294100

6.36154400

5.88867700

7.74925900

8.36611400

8.29756400

9.36523200

7.46494200

6.09291600

7.94713600

9.00621300

7.09638500

7.46146100

5.69959100

5.19695100

4.76763800

5.08481100

3.46540600

2.74091800

3.05773100

1.70226700

1.23151900

1.88173600 


$\begin{array}{lrrr}\mathrm{H} & 7.28349000 & 9.08680000 & -0.35855800 \\ \mathrm{C} & 7.61530900 & 7.04674700 & -0.89878400 \\ \mathrm{H} & 7.96559100 & 7.26275000 & -1.90303700 \\ \mathrm{C} & 7.57758800 & 5.70153400 & -0.46089400 \\ \mathrm{C} & 7.14707500 & 5.37070700 & 0.85551100 \\ \mathrm{C} & 7.98933400 & 4.65455100 & -1.31429800 \\ \mathrm{H} & 8.32849600 & 4.89165600 & -2.31785900 \\ \mathrm{C} & 7.97622900 & 3.36384400 & -0.87004300 \\ \mathrm{H} & 8.32008800 & 2.55801800 & -1.50496100 \\ \mathrm{C} & 7.58114500 & 3.11284500 & 0.45991200 \\ \mathrm{C} & 7.65094800 & 1.73040300 & 1.00944400 \\ \mathrm{C} & 7.62957500 & 0.59582100 & 0.20182300 \\ \mathrm{H} & 7.51784600 & 0.67942700 & -0.87202200 \\ \mathrm{C} & 7.72748300 & -0.65652600 & 0.79701500 \\ \mathrm{H} & 7.71111200 & -1.55222700 & 0.18585600 \\ \mathrm{C} & 7.84093400 & -0.74499200 & 2.17888400 \\ \mathrm{H} & 7.92410200 & -1.70143900 & 2.68040000 \\ \mathrm{C} & 7.83332600 & 0.43182000 & 2.91430400 \\ \mathrm{H} & 7.89887200 & 0.42104300 & 3.99719600 \\ \mathrm{Cl} & 9.85903600 & 3.85978600 & 3.29315600 \\ \mathrm{O} & 4.71363600 & 2.95578100 & 4.31455800 \\ \mathrm{~N} & 3.61773200 & 1.77361900 & 4.40436400 \\ \mathrm{O} & 2.70511300 & 2.13066800 & 5.06556500 \\ \mathrm{O} & 3.92841500 & 0.80219800 & 3.81349300 \\ & & & \end{array}$

$\begin{array}{lrrr}\text { Fe }^{\text {III }}(\mathbf{d p a}) & & & \\ \text { Complex 6 } & & & \\ 24 & & & \\ \mathrm{Fe} & -0.01707500 & -0.36297300 & -0.12979400 \\ \mathrm{Cl} & 0.20005500 & -0.24524600 & 2.17003300 \\ \mathrm{~N} & 1.54349900 & -1.62246900 & -0.25905800 \\ \mathrm{~N} & 1.39040400 & 1.01438600 & -0.13106200 \\ \mathrm{~N} & -1.39884800 & 1.01680600 & -0.10907300 \\ \mathrm{~N} & -1.57279100 & -1.58238200 & 0.17235300 \\ \mathrm{~N} & 0.00415500 & 2.91360900 & 0.16557100 \\ \mathrm{C} & 1.58909700 & -2.93537000 & -0.45683600 \\ \mathrm{H} & 0.64238800 & -3.44616400 & -0.56963600 \\ \mathrm{C} & 2.79253400 & -3.64505900 & -0.54693500 \\ \mathrm{H} & 2.76611600 & -4.71760000 & -0.69630000 \\ \mathrm{C} & 3.98876200 & -2.96539400 & -0.44838500 \\ \mathrm{H} & 4.93601200 & -3.49225900 & -0.50326000 \\ \mathrm{C} & 3.96281100 & -1.56481900 & -0.30271300 \\ \mathrm{C} & 2.70760600 & -0.94364900 & -0.22989200 \\ \mathrm{C} & 5.14266400 & -0.75103300 & -0.24071100 \\ \mathrm{H} & 6.11255400 & -1.23654900 & -0.28441900 \\ \mathrm{C} & 5.06474200 & 0.61633600 & -0.13195900\end{array}$




\begin{tabular}{lrrr}
$\mathrm{H}$ & 5.96940800 & 1.21284600 & -0.08624900 \\
$\mathrm{C}$ & 3.81039200 & 1.26314900 & -0.08599300 \\
$\mathrm{C}$ & 2.61929400 & 0.48959000 & -0.14957500 \\
$\mathrm{C}$ & 3.64611700 & 2.66940300 & 0.00265100 \\
$\mathrm{H}$ & 4.51585300 & 3.31785100 & 0.00886200 \\
$\mathrm{C}$ & 2.39518400 & 3.20031600 & 0.07371700 \\
$\mathrm{H}$ & 2.28129100 & 4.27344900 & 0.11388500 \\
$\mathrm{C}$ & 1.24128700 & 2.34969000 & 0.03353300 \\
$\mathrm{C}$ & -1.24614700 & 2.33356900 & -0.13728100 \\
$\mathrm{C}$ & -2.33557700 & 3.18343000 & -0.45053100 \\
$\mathrm{H}$ & -2.19192300 & 4.24969900 & -0.55842400 \\
$\mathrm{C}$ & -3.58274900 & 2.64607400 & -0.65320000 \\
$\mathrm{H}$ & -4.41642000 & 3.29361500 & -0.90416100 \\
$\mathrm{C}$ & -3.77977900 & 1.25636400 & -0.51699300 \\
$\mathrm{C}$ & -2.62855900 & 0.48737200 & -0.26993900 \\
$\mathrm{C}$ & -5.04689200 & 0.59847500 & -0.59969500 \\
$\mathrm{H}$ & -5.93087100 & 1.18830600 & -0.81594200 \\
$\mathrm{C}$ & -5.14686800 & -0.74076300 & -0.37467600 \\
$\mathrm{H}$ & -6.11612900 & -1.22758600 & -0.40459200 \\
$\mathrm{C}$ & -3.98792100 & -1.53791900 & -0.07447300 \\
$\mathrm{C}$ & -2.72867100 & -0.92586900 & -0.08629600 \\
$\mathrm{C}$ & -4.03264500 & -2.90408900 & 0.27379300 \\
$\mathrm{H}$ & -4.98111400 & -3.43122800 & 0.29255800 \\
$\mathrm{C}$ & -2.86401100 & -3.54049900 & 0.63084100 \\
$\mathrm{H}$ & -2.86479100 & -4.57355200 & 0.95661800 \\
$\mathrm{C}$ & -1.64847200 & -2.84198800 & 0.57501400 \\
$\mathrm{H}$ & -0.72704800 & -3.31181500 & 0.89419100 \\
$\mathrm{C}$ & -0.06036800 & 4.28023600 & 0.74433300 \\
$\mathrm{H}$ & -1.01689000 & 4.39840000 & 1.24562900 \\
$\mathrm{H}$ & 0.71124400 & 4.38119100 & 1.50376400 \\
$\mathrm{H}$ & 0.05688300 & 5.04373400 & -0.02870600 \\
$\mathrm{O}$ & -0.15107600 & -0.42813400 & -1.75002500 \\
& & & \\
\hline & & & \\
& & &
\end{tabular}

TS16

14

$\begin{array}{lrrr}\mathrm{Fe} & 0.03094900 & -0.57885700 & 0.10144000 \\ \mathrm{Cl} & 0.11430600 & -0.65135000 & 2.35234100 \\ \mathrm{~N} & 1.79486800 & -1.70125400 & 0.04625700 \\ \mathrm{~N} & 1.48299900 & 0.98065200 & 0.07090600 \\ \mathrm{~N} & -1.39733500 & 0.98861500 & 0.04182900 \\ \mathrm{~N} & -1.76857900 & -1.67221300 & 0.23210500 \\ \mathrm{~N} & 0.04312300 & 2.84178900 & 0.37761300 \\ \mathrm{C} & 1.90074100 & -3.01782300 & 0.03938500 \\ \mathrm{H} & 0.98394700 & -3.58605100 & 0.13917100 \\ \mathrm{C} & 3.13354500 & -3.66898100 & -0.09772300 \\ \mathrm{H} & 3.17013400 & -4.75134200 & -0.09318500\end{array}$




$\begin{array}{lrrr}\mathrm{C} & 4.27293900 & -2.91141200 & -0.23916700 \\ \mathrm{H} & 5.24344900 & -3.38446000 & -0.35075300 \\ \mathrm{C} & 4.18072500 & -1.50487200 & -0.24307900 \\ \mathrm{C} & 2.90084500 & -0.94008800 & -0.09504900 \\ \mathrm{C} & 5.31810600 & -0.64576500 & -0.39008400 \\ \mathrm{H} & 6.29850700 & -1.09460100 & -0.50807900 \\ \mathrm{C} & 5.16717700 & 0.70169400 & -0.38099100 \\ \mathrm{H} & 6.02575900 & 1.35543500 & -0.49336200 \\ \mathrm{C} & 3.87312000 & 1.29538800 & -0.22401800 \\ \mathrm{C} & 2.74000700 & 0.48699100 & -0.08747700 \\ \mathrm{C} & 3.65549600 & 2.68912200 & -0.21872200 \\ \mathrm{H} & 4.48921200 & 3.36706500 & -0.37057300 \\ \mathrm{C} & 2.39471900 & 3.18317900 & -0.04050000 \\ \mathrm{H} & 2.22757500 & 4.25012900 & -0.07427400 \\ \mathrm{C} & 1.30006500 & 2.29243200 & 0.13299300 \\ \mathrm{C} & -1.19065300 & 2.29541000 & 0.02410700 \\ \mathrm{C} & -2.23858900 & 3.18877700 & -0.33096900 \\ \mathrm{H} & -2.03957400 & 4.24785300 & -0.41898100 \\ \mathrm{C} & -3.48630500 & 2.70407000 & -0.60361700 \\ \mathrm{H} & -4.28489900 & 3.38191400 & -0.88786500 \\ \mathrm{C} & -3.73646100 & 1.31693400 & -0.52153100 \\ \mathrm{C} & -2.64069800 & 0.50353600 & -0.21400600 \\ \mathrm{C} & -5.02909900 & 0.73315900 & -0.72510000 \\ \mathrm{H} & -5.85775800 & 1.38621900 & -0.97841000 \\ \mathrm{C} & -5.22049400 & -0.60265400 & -0.58432000 \\ \mathrm{H} & -6.20406400 & -1.03974800 & -0.71947500 \\ \mathrm{C} & -4.12611900 & -1.46185500 & -0.24013300 \\ \mathrm{C} & -2.83998800 & -0.91380700 & -0.08668900 \\ \mathrm{C} & -4.26787600 & -2.84837400 & -0.02277500 \\ \mathrm{H} & -5.24344300 & -3.31103200 & -0.13435700 \\ \mathrm{C} & -3.17376700 & -3.59529500 & 0.34777200 \\ \mathrm{H} & -3.25470100 & -4.65690500 & 0.54642400 \\ \mathrm{C} & -1.92840000 & -2.96189500 & 0.46693300 \\ \mathrm{H} & -1.04988200 & -3.51665300 & 0.77528500 \\ \mathrm{C} & 0.01261900 & 4.17945000 & 0.98520400 \\ \mathrm{H} & -0.90819100 & 4.28510900 & 1.55774300 \\ \mathrm{H} & 0.84057800 & 4.26741800 & 1.68834400 \\ \mathrm{O} & 0.07484100 & 4.98656500 & 0.24751900 \\ \mathrm{O} & -0.05209000 & -0.77814800 & -1.52910200 \\ \mathrm{O} & -0.02085000 & 0.88699000 & -2.50243900 \\ \mathrm{H} & 1.20660500 & 1.08590500 & -3.00687500 \\ \mathrm{H} & 2.01170400 & 0.19046100 & -3.01268700 \\ \mathrm{H} & & & -3.40876200 \\ \mathrm{H} & & & \end{array}$

Int16

16 


\begin{tabular}{|c|c|c|c|}
\hline $\mathrm{Fe}$ & 0.09825700 & -0.53973400 & 0.33153300 \\
\hline $\mathrm{Cl}$ & -0.04874500 & -0.83084800 & 2.57383400 \\
\hline $\mathrm{N}$ & 1.94702000 & -1.65809700 & 0.32821200 \\
\hline $\mathrm{N}$ & 1.58082900 & 1.02190400 & 0.22744400 \\
\hline $\mathrm{N}$ & -1.30976700 & 1.07420400 & 0.06127600 \\
\hline $\mathrm{N}$ & -1.76887700 & -1.58745600 & 0.07473300 \\
\hline $\mathrm{N}$ & 0.14715300 & 2.87640800 & 0.56216200 \\
\hline $\mathrm{C}$ & 2.09444700 & -2.96292400 & 0.46424400 \\
\hline $\mathrm{H}$ & 1.21067700 & -3.52794200 & 0.74003800 \\
\hline $\mathrm{C}$ & 3.32823600 & -3.60177300 & 0.27622500 \\
\hline $\mathrm{H}$ & 3.40086600 & -4.67566200 & 0.39669100 \\
\hline $\mathrm{C}$ & 4.42420600 & -2.84119400 & -0.05959700 \\
\hline $\mathrm{H}$ & 5.39320600 & -3.30429300 & -0.21757800 \\
\hline $\mathrm{C}$ & 4.29352000 & -1.44254900 & -0.18836700 \\
\hline $\mathrm{C}$ & 3.01626800 & -0.89204700 & 0.02222400 \\
\hline $\mathrm{C}$ & 5.39120400 & -0.57782100 & -0.50608100 \\
\hline $\mathrm{H}$ & 6.36785600 & -1.01625600 & -0.68138500 \\
\hline $\mathrm{C}$ & 5.20983700 & 0.76426500 & -0.57478100 \\
\hline $\mathrm{H}$ & 6.03974700 & 1.42350500 & -0.80706100 \\
\hline $\mathrm{C}$ & 3.92369900 & 1.34705200 & -0.32905600 \\
\hline $\mathrm{C}$ & 2.82239800 & 0.53081500 & -0.04561400 \\
\hline $\mathrm{C}$ & 3.69443900 & 2.73928000 & -0.34729600 \\
\hline $\mathrm{H}$ & 4.50471300 & 3.41710900 & -0.59649700 \\
\hline $\mathrm{C}$ & 2.45171100 & 3.22869700 & -0.06117300 \\
\hline $\mathrm{H}$ & 2.26616600 & 4.29308300 & -0.10081500 \\
\hline $\mathrm{C}$ & 1.39338400 & 2.33157700 & 0.23802500 \\
\hline $\mathrm{C}$ & -1.08926700 & 2.38110500 & 0.15858900 \\
\hline $\mathrm{C}$ & -2.11746800 & 3.31784500 & -0.13384000 \\
\hline $\mathrm{H}$ & -1.90583800 & 4.37753400 & -0.11622300 \\
\hline $\mathrm{C}$ & -3.35925600 & 2.87782400 & -0.49236600 \\
\hline $\mathrm{H}$ & -4.13844000 & 3.59037500 & -0.74373000 \\
\hline $\mathrm{C}$ & -3.63013100 & 1.49370700 & -0.54387700 \\
\hline $\mathrm{C}$ & -2.56414000 & 0.63478400 & -0.25756900 \\
\hline $\mathrm{C}$ & -4.91980900 & 0.96095100 & -0.86887100 \\
\hline $\mathrm{H}$ & -5.72037400 & 1.65428600 & -1.10450400 \\
\hline $\mathrm{C}$ & -5.13879800 & -0.37728700 & -0.87621100 \\
\hline $\mathrm{H}$ & -6.11691600 & -0.77907600 & -1.11812800 \\
\hline $\mathrm{C}$ & -4.07997600 & -1.28613300 & -0.55177900 \\
\hline $\mathrm{C}$ & -2.80185300 & -0.78179300 & -0.25115800 \\
\hline $\mathrm{C}$ & -4.24934300 & -2.68565100 & -0.50475400 \\
\hline $\mathrm{H}$ & -5.21977100 & -3.11487500 & -0.73347300 \\
\hline $\mathrm{C}$ & -3.18727900 & -3.49062400 & -0.16379800 \\
\hline $\mathrm{H}$ & -3.28820900 & -4.56771800 & -0.11206700 \\
\hline $\mathrm{C}$ & -1.95122200 & -2.89415700 & 0.12288200 \\
\hline $\mathrm{H}$ & -1.09408500 & -3.49774600 & 0.40033600 \\
\hline $\mathrm{C}$ & 0.15628500 & 4.18790200 & 1.22244700 \\
\hline
\end{tabular}




$\begin{array}{lrrr}\mathrm{H} & -0.72800200 & 4.27103900 & 1.85432500 \\ \mathrm{H} & 1.02834700 & 4.24458100 & 1.87348100 \\ \mathrm{H} & 0.17795300 & 5.02671600 & 0.51808100 \\ \mathrm{O} & 0.38953500 & -0.97356500 & -1.54850500 \\ \mathrm{O} & -0.66631100 & -0.60304000 & -2.36480500 \\ \mathrm{~N} & -0.50713400 & 0.73358200 & -2.83748800 \\ \mathrm{O} & -1.45448600 & 1.07644400 & -3.49072400 \\ \mathrm{O} & 0.49115900 & 1.32735200 & -2.53393500\end{array}$

\section{TS26}

16

$\begin{array}{lrrr}\mathrm{Fe} & -0.16094500 & -0.17178200 & 0.81638100 \\ \mathrm{Cl} & -0.15153900 & -0.13871100 & 3.08570600 \\ \mathrm{~N} & 1.85067200 & -1.00118100 & 0.71601600 \\ \mathrm{~N} & 1.03786800 & 1.53146000 & 0.18774500 \\ \mathrm{~N} & -1.78660900 & 0.99719700 & -0.01058000 \\ \mathrm{~N} & -1.79052100 & -1.61310600 & 0.73251000 \\ \mathrm{~N} & -0.72540100 & 3.11080000 & 0.09744000 \\ \mathrm{C} & 2.21024300 & -2.24191900 & 0.99296500 \\ \mathrm{H} & 1.41436700 & -2.93094000 & 1.25325200 \\ \mathrm{C} & 3.54615800 & -2.66888900 & 0.95223300 \\ \mathrm{H} & 3.78844600 & -3.69875700 & 1.18418200 \\ \mathrm{C} & 4.52190300 & -1.75981600 & 0.61671500 \\ \mathrm{H} & 5.56591400 & -2.05464400 & 0.57696000 \\ \mathrm{C} & 4.16525600 & -0.42554700 & 0.32740100 \\ \mathrm{C} & 2.79801800 & -0.09455200 & 0.38822500 \\ \mathrm{C} & 5.12547700 & 0.58370000 & -0.00727600 \\ \mathrm{H} & 6.17450800 & 0.31147400 & -0.05528100 \\ \mathrm{C} & 4.72652000 & 1.85777900 & -0.24469000 \\ \mathrm{H} & 5.45119400 & 2.62856700 & -0.48604600 \\ \mathrm{C} & 3.34168700 & 2.21925500 & -0.17864200 \\ \mathrm{C} & 2.37049100 & 1.25390200 & 0.11381900 \\ \mathrm{C} & 2.88064600 & 3.53561600 & -0.39685100 \\ \mathrm{H} & 3.58767200 & 4.31968500 & -0.64941800 \\ \mathrm{C} & 1.54787800 & 3.81855700 & -0.30299500 \\ \mathrm{H} & 1.19596900 & 4.82182500 & -0.49781300 \\ \mathrm{C} & 0.62630800 & 2.77879600 & 0.00131600 \\ \mathrm{C} & -1.79689000 & 2.29279100 & -0.28023900 \\ \mathrm{C} & -2.89944800 & 2.89851500 & -0.93932600 \\ \mathrm{H} & -2.86867900 & 3.94797400 & -1.19952100 \\ \mathrm{C} & -3.98394700 & 2.13798800 & -1.27782800 \\ \mathrm{H} & -4.82539800 & 2.58742400 & -1.79595800 \\ \mathrm{C} & -4.01135900 & 0.76326400 & -0.95709000 \\ \mathrm{C} & -2.87482300 & 0.24096100 & -0.32565700 \\ \mathrm{C} & -5.13564400 & -0.08153300 & -1.23403400 \\ \mathrm{H} & -5.99356900 & 0.34772700 & -1.74117900\end{array}$




$\begin{array}{lrrr}\mathrm{C} & -5.13630200 & -1.38341700 & -0.85444900 \\ \mathrm{H} & -5.99411500 & -2.01791700 & -1.05119400 \\ \mathrm{C} & -4.00932000 & -1.94217700 & -0.16677700 \\ \mathrm{C} & -2.87677300 & -1.14363700 & 0.07854900 \\ \mathrm{C} & -3.98019000 & -3.27038000 & 0.30916100 \\ \mathrm{H} & -4.83366000 & -3.91888200 & 0.13718800 \\ \mathrm{C} & -2.88164600 & -3.72158600 & 1.00230200 \\ \mathrm{H} & -2.83850300 & -4.72842400 & 1.39942800 \\ \mathrm{C} & -1.79946300 & -2.84948000 & 1.19657500 \\ \mathrm{H} & -0.92434800 & -3.16065400 & 1.75767700 \\ \mathrm{C} & -1.05421500 & 4.49564500 & 0.45449600 \\ \mathrm{H} & -2.03807600 & 4.51243000 & 0.92277800 \\ \mathrm{H} & -0.33660300 & 4.85022900 & 1.19467200 \\ \mathrm{H} & -1.05413300 & 5.17621000 & -0.40447100 \\ \mathrm{O} & -0.08252600 & -1.15630600 & -1.21232600 \\ \mathrm{O} & 0.85636200 & -0.66643400 & -1.94691100 \\ \mathrm{~N} & 1.73746700 & -1.86754100 & -2.51631900 \\ \mathrm{O} & 2.62416200 & -1.44173200 & -3.17749500 \\ \mathrm{O} & 1.37056800 & -2.93637400 & -2.17076700\end{array}$




\section{REFERENCES}

1. Marsac, R.; Real, F.; Banik, N. L.; Pedrot, M.; Pourret, O.; Vallet, V., Aqueous chemistry of Ce(IV): estimations using actinide analogues. Dalton Trans. 2017, 46, 13553-13561.

2. Karkas, M. D.; Verho, O.; Johnston, E. V.; Akermark, B., Artificial Photosynthesis: Molecular Systems for Catalytic Water Oxidation. Chem. Rev. 2014, 114, 11863-12001.

3. Andrae, D.; Häußermann, U.; Dolg, M.; Stoll, H.; Preuß, H., Energy-adjustedab initio pseudopotentials for the second and third row transition elements. Theor. Chim. Acta. 1990, 77, $123-141$.

4. Marenich, A. V.; Cramer, C. J.; Truhlar, D. G., Universal Solvation Model Based on Solute Electron Density and on a Continuum Model of the Solvent Defined by the Bulk Dielectric Constant and Atomic Surface Tensions. J. Phys. Chem. B 2009, 113, 6378-6396. 